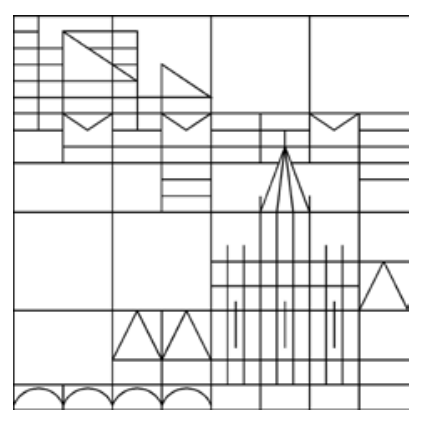

Maximal regularity for the thermoelastic plate equations with free boundary conditions

\author{
Robert Denk \\ Yoshihiro Shibata
}

Konstanzer Schriften in Mathematik

Nr. 352, März 2016

ISSN 1430-3558

Konstanzer Online-Publikations-System (KOPS)

URL: http://nbn-resolving.de/urn:nbn:de:bsz:352-0-325845 



\title{
Maximal regularity for the thermoelastic plate equations with free boundary conditions
}

\author{
Robert Denk* and Yoshihiro Shibata ${ }^{\dagger}$
}

February 25, 2016

\begin{abstract}
We consider the linear thermoelastic plate equations with free boundary conditions in the $L_{p}$ in time and $L_{q}$ in space setting. We obtain unique solvability with optimal regularity for the inhomogeneous problem in a uniform $C^{4}$-domain, which includes the cases of a bounded domain and of an exterior domain with $C^{4}$-boundary. Moreover, we prove uniform a priori-estimates for the solution. The proof is based on the existence of $\mathcal{R}$-bounded solution operators of the corresponding generalized resolvent problem which is shown with the help of an operator-valued Fourier multiplier theorem due to Weis.
\end{abstract}

\section{InTRODUCTION}

Let $\Omega$ be a domain in the $N$-dimensional Euclidean space $\mathbb{R}^{N}$ with boundary $\Gamma$. In the present paper we consider the linearized thermoelastic plate equations given by

$$
\begin{aligned}
& u_{t t}+\Delta^{2} u+\Delta \theta=f_{1} \quad \text { in }(0, \infty) \times \Omega \text {, } \\
& \theta_{t}-\Delta \theta-\Delta u_{t}=f_{2} \quad \text { in }(0, \infty) \times \Omega
\end{aligned}
$$

with initial conditions

$$
\begin{aligned}
\left.u\right|_{t=0}=u_{0} & \text { in } \Omega, \\
\left.u_{t}\right|_{t=0}=u_{1} & \text { in } \Omega, \\
\left.\theta\right|_{t=0}=\theta_{0} & \text { in } \Omega .
\end{aligned}
$$

In (1-1)-(1-2), we omit all physical constants for simplicity of presentation. These equations model the behaviour of a thin plate with the elastic properties being influenced by the temperature (see, e.g., [14]). In (1-1), $u=u(t, x)$ stands for the vertical displacement at time $t$ and at the point $x=\left(x_{1}, \ldots, x_{N}\right) \in \Omega$ while $\theta=\theta(t, x)$ describes the temperature relative to a constant reference temperature. For (1-1), several boundary conditions are of interest, see, e.g. [15] for a survey on physically relevant boundary conditions. In the present paper, we consider free boundary conditions which are given by

$$
\begin{aligned}
\Delta u-(1-\beta) \Delta^{\prime} u+\theta & =g_{1} & & \text { on }(0, \infty) \times \Gamma, \\
\partial_{\nu}\left(\Delta u+(1-\beta) \Delta^{\prime} u+\theta\right) & =g_{2} & & \text { on }(0, \infty) \times \Gamma, \\
\partial_{\nu} \theta & =g_{3} & & \text { on }(0, \infty) \times \Gamma .
\end{aligned}
$$

${ }^{*}$ Universität Konstanz, Fachbereich für Mathematik und Statistik, 78457 Konstanz, Germany

e-mail address: robert.denk@uni-konstanz.de

${ }^{\dagger}$ Department of Mathematics and Research Institute of Science and Engineering

Waseda University, Ohkubo 3-4-1, Shinjuku-ku, Tokyo 169-8555, Japan.

e-mail address: yshibata@waseda.jp

Partially supported by JSPS Grant-in-aid for Scientific Research (S) \# 24224004 and Top Global University Project

Subjectclass(2010) : 35K35; 35J40; 42B15

Keywords: Thermoelastic plate equations; generation of analytic semigroups; maximal $L_{p}$ - $L_{q}$ regularity; $\mathcal{R}$-bounded solution operator, operator-valued Fourier multipliers 
In (1-3), $\beta \in[0,1)$ is a parameter which is fixed throughout this paper, $\Delta$ and $\Delta^{\prime}$ stand for the Laplace operator in $\Omega$ and the Laplace-Beltrami operator on $\Gamma$, respectively, and $\partial_{\nu}$ denotes the derivative in outer normal direction. Note that the term $\theta$ can be omitted in the second line of (1-3) if we replace $g_{2}$ by $g_{2}-g_{3}$.

There is a rich literature on the thermoelastic plate equations under various kinds of boundary conditions. Exponential stability of the associated semigroup in $L_{2}$ (in the case of a bounded domain) has been proved by Kim [12], Munos Rivera and Racke [22], Liu and Zheng [21], Lasiecka and Triggiani [15] -[16], and Shibata [26]. For a survey on general von Karman evolution equations, we refer to Chuesov and Lasiecka [2]. It turns out that the generated semigroup is even analytic, see also Liu and Renardy [20], Liu and Liu [18], and Liu and Yong [19] in the $L_{2}$-setting. This means that the effect from the heat equation in $\theta$ is strong enough to obtain analytic behaviour of the whole system although the first equation in (1-1) is a simply dispersive equation (the product of two Schrödinger equations) with respect to $u$.

Most of the results mentioned above are obtained in an $L_{2}$-setting, where energy methods are available. However, as the original equations modelling thermoelastic plates are non-linear, an $L_{p}$-approach is also relevant in order to handle equations with low regularity of the data. Therefore, several results on (1-1) in $L_{p}$-spaces were obtained. In the whole-space case, analyticity of the generated semigroup in $L_{p}$ was shown by Denk and Racke [4]. In the case of the half-space and of bounded domains, equations (1-1) with Dirichlet boundary conditions

$$
u=\partial_{\nu} u=\theta=0 \quad \text { on }(0, \infty) \times \Gamma
$$

were studied by Naito and Shibata [24] and by Naito [23]. It was shown that in $L_{p}$ an analytic $C^{0}$ semigroup is generated and that even maximal $L_{p}$ - $L_{q}$-regularity holds which is the key property for the analysis of the non-linear equations. By Denk, Racke and Shibata [5], [6] energy estimates for the generated semigroup in $L_{q}$ were shown.

The proof of maximal $L_{p}$-regularity for the linearized system and a rather complete analysis of the non-linear thermoelastic plate equations can be found in a recent paper by Lasiecka and Wilke [17]. In that paper, the boundary conditions

$$
u=\Delta u=\theta=0 \quad \text { on }(0, \infty) \times \Gamma
$$

are studied. From a mathematical point of view, these boundary conditions are easier to handle. This is due to the fact that the operator $\Delta^{2}$ appearing in the first line of (1-1) can then be interpreted as the square of the Dirichlet-Laplace operator, and solvability of (1-1) can be shown by abstract operatortheoretic methods. For the boundary conditions (1-3) studied in the present paper, such an abstract approach seems to be not available, and one needs a thorough analysis of the (localized) solution operators.

The purpose of this paper is to prove maximal $L_{p}$ - $L_{q}$-regularity of the initial boundary value problem (1-1)-(1-3). In our approach, setting $v=u_{t}$ we rewrite (1-1) as a first-order system acting on $U=$ $(u, v, \theta)^{\top}$, where $M^{\top}$ denotes the transposed of $M$, and being of the form

$$
U_{t}-A(D) U=F \quad \text { in }(0, T) \times \Omega, \quad B(D) U=G \quad \text { on }(0, T) \times \Gamma,\left.\quad U\right|_{t=0}=U_{0} \quad \text { in } \Omega
$$

with operator-matrices $A(D)$ and $B(D)$ being defined by

$$
A(D):=\left(\begin{array}{ccc}
0 & 1 & 0 \\
-\Delta^{2} & 0 & -\Delta \\
0 & \Delta & \Delta
\end{array}\right), \quad B(D):=\left(\begin{array}{ccc}
\Delta-(1-\beta) \Delta^{\prime} & 0 & 1 \\
\partial_{\nu}\left(\Delta+(1-\beta) \Delta^{\prime}\right) & 0 & 0 \\
0 & 0 & \partial_{\nu}
\end{array}\right) .
$$

Setting $F=\left(0, f_{1}, f_{2}\right)^{\top}, U_{0}=\left(u_{0}, u_{1}, \theta_{0}\right)^{\top}, G=\left(g_{1}, g_{2}, g_{3}\right)^{\top}$, and $U=\left(u, u_{t}, \theta\right)^{\top}$ in (1-4) represents the equations (1-1)-(1-3). To prove maximal $L_{p}$ - $L_{q}$-regularity of problem (1-4), we prove the existence of an $\mathcal{R}$-bounded solution operator of the problem:

$$
\lambda U-A(D) U=F \quad \text { in } \Omega, \quad B(D) U=G \quad \text { on } \Gamma
$$

with $F=\left(0, f_{1}, f_{2}\right)^{\top} \in L_{q}(\Omega)^{2}$ and $G=\left(g_{1}, g_{2}, g_{3}\right)^{\top} \in H_{q}^{2}(\Omega) \times H_{q}^{1}(\Omega)^{2}$, which is the generalized resolvent problem corresponding to problem (1-4). 
To state the main results pricesely, at this point we introduce several symbols used throughout the paper. $\mathbb{N}, \mathbb{R}$, and $\mathbb{C}$ denote the sets of all natural numbers, real numbers, and complex numbers, respectively. Set $\mathbb{N}_{0}=\mathbb{N} \cup\{0\}$. For any multi-index $\kappa=\left(\kappa_{1}, \ldots, \kappa_{N}\right) \in \mathbb{N}_{0}^{N}$, we write $|\kappa|=\kappa_{1}+\cdots+\kappa_{N}$ and $\partial_{x}^{\kappa}=\partial_{1}^{\kappa_{1}} \cdots \partial_{N}^{\kappa_{N}}$ with $\partial_{i}=\partial / \partial x_{i}$. For any scalar function $f$ and vector-valued function $\mathbf{g}=$ $\left(g_{1}, \ldots, g_{k}\right)$, let

$$
\begin{aligned}
& \nabla f=\left(\partial_{1} f, \ldots, \partial_{N} f\right), \quad \nabla^{\ell} f=\left(\partial_{x}^{\alpha} f|| \alpha \mid=\ell\right), \\
& \nabla \mathbf{g}=\left(\partial_{i} g_{j} \mid i=1, \ldots, N, j=1, \ldots, k\right), \quad \nabla^{\ell} \mathbf{g}=\left(\nabla^{\ell} g_{1}, \ldots, \nabla^{\ell} g_{k}\right) .
\end{aligned}
$$

For any domain $D$, let $L_{q}(D), H_{q}^{m}(D)(m \in \mathbb{N})$ and $B_{q, p}^{s}(D)(s \in(0, \infty) \backslash \mathbb{N})$ be the Lebesgue space, Sobolev space and Besov space, while $\|\cdot\|_{L_{q}(D)},\|\cdot\|_{H_{q}^{m}(D)}$ and $\|\cdot\|_{B_{q, p}^{s}(D)}$ denote their norms, respectively. We write $H_{q}^{0}(D)=L_{q}(D)$ and $B_{q q}^{s}(D)=W_{q}^{s}(D)$. Let $X$ and $Y$ be Banach spaces, and let $\mathcal{L}(X, Y)$ be the space of all bounded linear operators from $X$ to $Y$. We use the abbreviation $\mathcal{L}(X)=\mathcal{L}(X, X)$. For an interval $J=(0, T)$ with $T \in(0, \infty], L_{p}(J, X)$ denotes the $X$-valued Lebesgue space and $H_{p}^{m}(J, X)$ $(m \in \mathbb{N})$ the $X$-valued Sobolev space, while $\|\cdot\|_{L_{p}(J, X)}$ and $\|\cdot\|_{H_{p}^{m}(J, X)}$ denote their norms, respectively. For any domain $V$ in $\mathbb{C}, \mathcal{C}(V, X)$ denotes the set of all $X$-valued functions $f=f(\lambda)$ defined for $\lambda=$ $\gamma+i \tau \in V$ which are continuously differentiable with respect to $\tau$ when $\lambda \in V$. Let $\Sigma_{\vartheta}$ and $\Sigma_{\vartheta, \lambda_{0}}$ be the sets in $\mathbb{C}$ defined by

$$
\Sigma_{\vartheta}:=\{\lambda \in \mathbb{C} \backslash\{0\}|| \arg \lambda \mid<\vartheta\}, \quad \Sigma_{\vartheta, \lambda_{0}}:=\left\{\lambda \in \Sigma_{\vartheta}|| \lambda \mid \geq \lambda_{0}\right\}
$$

for any $0<\vartheta \leq \pi$ and $\lambda_{0}>0$. Let $X^{d}=\left\{\mathbf{f}=\left(f_{1}, \ldots, f_{d}\right)^{\top} \mid f_{i} \in X(i=1, \ldots, d)\right\}$, while the norm of $X^{d}$ is written by $\|\cdot\|_{X}$ instead of $\|\cdot\|_{X^{d}}$ for short. In particular, we write

$$
\|G\|_{H_{q}^{m}(D) \times H_{q}^{\ell}(D)^{2}}=\left\|g_{1}\right\|_{H_{q}^{m}(D)}+\left\|\left(g_{2}, g_{3}\right)\right\|_{H_{q}^{\ell}(D)} \quad \text { for } G=\left(g_{1}, g_{2}, g_{3}\right)^{\top} \in H_{q}^{m}(D) \times H_{q}^{\ell}(D)^{2} .
$$

Let

$$
\begin{aligned}
& \mathbb{G}_{q}(D):=\left\{(F, G) \mid F=\left(0, f_{1}, f_{2}\right)^{\top}, \quad\left(f_{1}, f_{2}\right) \in L_{q}(D)^{2}, G=\left(g_{1}, g_{2}, g_{3}\right)^{\top} \in H_{q}^{2}(D) \times H_{q}^{1}(D)^{2}\right\}, \\
& \|(F, G)\|_{\mathbb{G}_{q}(D)}:=\left\|\left(f_{1}, f_{2}\right)\right\|_{L_{q}(D)}+\|G\|_{H_{q}^{2}(D) \times H_{q}^{1}(D)^{2}}, \\
& \mathcal{X}_{q}(\Omega):=\left\{\mathcal{H}=\left(F^{\prime}, G, G^{\prime}, g_{1}^{\prime \prime}\right) \mid F^{\prime}=\left(f_{1}, f_{2}\right)^{\top} \in L_{q}(\Omega)^{2},\right. \\
& \left.\quad G=\left(g_{1}, g_{2}, g_{3}\right)^{\top} \in H_{q}^{2}(\Omega) \times H_{q}^{1}(\Omega)^{2}, G^{\prime}=\left(g_{1}^{\prime}, g_{2}^{\prime}, g_{3}^{\prime}\right)^{\top} \in H_{q}^{1}(\Omega) \times L_{q}(\Omega)^{2}, \quad g_{1}^{\prime \prime} \in L_{q}(\Omega)\right\}, \\
& \|\mathcal{H}\|_{\mathcal{X}_{q}(D)}:=\left\|\left(f_{1}, f_{2}\right)\right\|_{L_{q}(D)}+\|G\|_{H_{q}^{2}(D) \times H_{q}^{1}(D)}+\left\|G^{\prime}\right\|_{H_{q}^{1}(D) \times L_{q}(D)^{2}}+\left\|g_{1}^{\prime \prime}\right\|_{L_{q}(D)} .
\end{aligned}
$$

For any $\lambda \in \mathbb{C}$ and $(F, G) \in \mathbb{G}_{q}(D)$, let $H_{\lambda}(F, G):=\left(f_{1}, f_{2}, G, \lambda^{1 / 2} G, \lambda g_{1}\right)$ with $F=\left(0, f_{1}, f_{2}\right)^{\top}$ and $G=\left(g_{1}, g_{2}, g_{3}\right)^{\top}$. In particular, $G^{\prime}$ and $g_{1}^{\prime \prime}$ are the corresponding variables to $\lambda^{1 / 2} G$ and $\lambda g_{1}$. For any exponent $q \in(1, \infty)$, let $q^{\prime}=q /(q-1)$ be the dual exponent of $q$. The letters $C$ and $c$ denote generic positive constants and the constant $C_{a, b, \ldots}$ depends on $a, b, \ldots$ The values of the constants $C, c$ and $C_{a, b, \ldots}$ may change from line to line.

Next, we introduce two definitions (see, e.g., [3], [13]).

Definition 1.1. A family $\mathcal{T} \subset \mathcal{L}(X, Y)$ of operators is called $\mathcal{R}$-bounded if for one (and then all) $p \in[1, \infty)$ there exists a constant $C>0$ such that for all $m \in \mathbb{N},\left(T_{k}\right)_{k=1, \ldots, m} \subset \mathcal{T}$, and $\left(x_{k}\right)_{k=1, \ldots, m} \subset X$ we have

$$
\left\|\sum_{k=1}^{m} r_{k} T_{k} x_{k}\right\|_{L_{p}([0,1], Y)} \leq C\left\|\sum_{k=1}^{m} r_{k} x_{k}\right\|_{L_{p}([0,1], X)} .
$$

Here the Rademacher functions $r_{k}, k \in \mathbb{N}$, are given by $r_{k}:[0,1] \rightarrow\{-1,1\}, t \mapsto \operatorname{sign}\left(\sin \left(2^{k} \pi t\right)\right)$. The smallest such $C$ is called the $\mathcal{R}$-bound of $\mathcal{T}$ on $\mathcal{L}(X, Y)$ which is written by $\mathcal{R}_{\mathcal{L}(X, Y)}(\mathcal{T})$ in what follows. Note that we omit the dependence on $p$ in the notation of the $\mathcal{R}$-bound.

Definition 1.2. A domain $\Omega$ is called a uniform $C^{4}$-domain if there exist positive constants $\alpha, \beta$ and $K$ such that for any $x_{0} \in \Gamma$ there exist a coordinate number $j$ and a $C^{4}$-function $h\left(x^{\prime}\right)$ defined on $B_{\alpha}^{\prime}\left(x_{0}^{\prime}\right)$ such that $\|h\|_{H_{\infty}^{4}\left(B_{\alpha}^{\prime}\left(x_{0}^{\prime}\right)\right)} \leq K$ and

$$
\begin{aligned}
& \Omega \cap B_{\beta}\left(x_{0}\right)=\left\{x \in \mathbb{R}^{N} \mid x_{j}>h\left(x^{\prime}\right)\left(x^{\prime} \in B_{\alpha}^{\prime}\left(x_{0}^{\prime}\right)\right)\right\} \cap B_{\beta}\left(x_{0}\right), \\
& \Gamma \cap B_{\beta}\left(x_{0}\right)=\left\{x \in \mathbb{R}^{N} \mid x_{j}=h\left(x^{\prime}\right)\left(x^{\prime} \in B_{\alpha}^{\prime}\left(x_{0}^{\prime}\right)\right)\right\} \cap B_{\beta}\left(x_{0}\right) .
\end{aligned}
$$


Here, $x^{\prime}$ has been defined by $x^{\prime}=\left(x_{1}, \ldots, x_{j-1}, x_{j+1}, \ldots, x_{N}\right)$ for $x=\left(x_{1}, \ldots, x_{N}\right)$,

$$
B_{\alpha}^{\prime}\left(x_{0}^{\prime}\right)=\left\{x^{\prime} \in \mathbb{R}^{N-1}|| x^{\prime}-x_{0}^{\prime} \mid<\alpha\right\}, \quad B_{\beta}\left(x_{0}\right)=\left\{x \in \mathbb{R}^{N}|| x-x_{0} \mid<\beta\right\} .
$$

In what follows, $\Omega$ is assumed to be a uniform $C^{4}$-domain. Let $\iota: L_{1, \text { loc }}(\Omega) \rightarrow L_{1, \text { loc }}\left(\mathbb{R}^{N}\right)$ be an extension operator possessing the following properties:

(e-1) For any $1<q<\infty$ and $f \in H_{q}^{i}(\Omega)$ we have $\iota f \in H_{q}^{i}\left(\mathbb{R}^{N}\right), \iota f=f$ in $\Omega$ and $\|\iota f\|_{H_{q}^{i}\left(\mathbb{R}^{N}\right)} \leq$ $C_{q}\|f\|_{H_{q}^{i}(\Omega)}$ for $i=0,, \ldots, 4$.

(e-2) For any $1<q<\infty$ and $f \in H_{q}^{1}(\Omega),\left\|(1-\Delta)^{-1 / 2} \iota(\nabla f)\right\|_{L_{q}\left(\mathbb{R}^{N}\right)} \leq C_{q}\|f\|_{L_{q}(\Omega)}$.

Here, $(1-\Delta)^{-1 / 2}$ is the operator defined by $(1-\Delta)^{-1 / 2} f=\mathscr{F}^{-1}\left[\left(1+|\xi|^{2}\right)^{-1 / 2} \mathscr{F} f\right]$ with the help of the Fourier transform $\mathscr{F}$ and its inverse transform $\mathscr{F}^{-1}$ defined by

$$
(\mathscr{F} \varphi)(\xi)=\int_{\mathbb{R}^{n}} \varphi(x) e^{-i x \xi} d x \quad\left(\xi \in \mathbb{R}^{n}\right), \quad\left(\mathscr{F}^{-1} \varphi\right)(x)=\frac{1}{(2 \pi)^{n}} \int_{\mathbb{R}^{n}} \varphi(\xi) e^{i x \xi} d \xi \quad\left(x \in \mathbb{R}^{n}\right) .
$$

For the existence of such an extension operator, we refer, e.g., to Schade and Shibata [25, Appendix A]. In what follows, such $\iota$ is fixed. Let $\mathbf{W}_{q}^{-1}(\Omega)$ be the space defined by

$$
\mathbf{W}_{q}^{-1}(\Omega)=\left\{f \in L_{1, \mathrm{loc}}(\Omega) \mid\|f\|_{\mathbf{W}_{q}^{-1}(\Omega)}=\left\|(1-\Delta)^{-1 / 2} \iota f\right\|_{L_{q}\left(\mathbb{R}^{N}\right)}<\infty\right\} .
$$

Finally, we state the main results of this paper.

Theorem 1.3 (Maximal $L_{p}-L_{q}$-regularity). Let $T>0$. Let $1<p, q<\infty$. Assume that $\Omega$ is a uniform $C^{4}$-domain in $\mathbb{R}^{N}$. Then, there exists a number $\lambda_{1}$ such that for any initial data $U_{0}=\left(u_{0}, u_{1}, \theta_{0}\right)^{\top} \in$ $B_{q, p}^{4-2 / p}(\Omega) \times B_{q, p}^{2-2 / p}(\Omega)^{2}$, right-hand side $F=\left(0, f_{1}, f_{2}\right)^{\top}$ with $\left(f_{1}, f_{2}\right)^{\top} \in L_{p}\left((0, T), L_{q}(\Omega)^{2}\right)$ and boundary data $G=\left(g_{1}, g_{2}, g_{3}\right)^{\top}$ with

$$
G \in H_{p}^{1}\left((0, T), L_{q}(\Omega) \times \mathbf{W}_{q}^{-1}(\Omega)^{2}\right) \cap L_{p}\left((0, T), H_{q}^{2}(\Omega) \times H_{q}^{1}(\Omega)^{2}\right)
$$

satisfying the compatibility condition: $\left.G\right|_{t=0}=B(D) U_{0}$ on $\Omega$, problem (1-4) admits a unique solution $U=\left(u, u_{t}, \theta\right)^{\top}$ with

$$
u \in \bigcap_{\ell=0}^{2} H_{p}^{\ell}\left((0, T), H_{q}^{4-2 \ell}(\Omega)\right), \quad \theta \in \bigcap_{\ell=0}^{1} H_{p}^{\ell}\left((0, T), H_{q}^{2-2 \ell}(\Omega)\right)
$$

possessing the estimate:

$$
\begin{aligned}
& \sum_{\ell=0}^{2}\left\|\partial_{t}^{\ell} u\right\|_{L_{p}\left((0, T), H_{q}^{4-2 \ell}(\Omega)\right)}+\sum_{\ell=0}^{1}\left\|\partial_{t}^{\ell} \theta\right\|_{L_{p}\left((0, T), H_{q}^{2-2 \ell}(\Omega)\right)} \leq e^{\gamma T}\left\{\left\|u_{0}\right\|_{B_{q, p}^{4-2 / p}(\Omega)}+\left\|\theta_{0}\right\|_{B_{q, p}^{2-2 / p}(\Omega)}\right. \\
& \left.+\left\|\left(f_{1}, f_{2}\right)\right\|_{L_{p}\left((0, T), L_{q}(\Omega)^{2}\right)}+\|G\|_{L_{p}\left((0, T), H_{q}^{2}(\Omega) \times H_{q}^{1}(\Omega)^{2}\right)}+\left\|\partial_{t} G\right\|_{L_{p}\left((0, T), L_{q}(\Omega) \times \mathbf{W}_{q}^{-1}(\Omega)^{2}\right)}\right\}
\end{aligned}
$$

with some positive constant $\gamma>0$ independent of $T$.

Concerning the compatibility conditions, we remark that $\left.G\right|_{t=0}$ and $B(D) U_{0}$ both belong to the space $B_{q p}^{2-2 / p}(\Omega) \times\left(B_{p q}^{1-2 / p}(\Omega)\right)^{2}$ in the case $p>2$. For simplicity, we assume that the compatibility conditions holds in $\Omega$. In view of the nonlinear equation, we typically assume $2<p<\infty, N<q<\infty$, and $2 / p+N / q<1$. In this case, the traces of $\left.G\right|_{t=0}$ and $B(D) U_{0}$ on the boundary exist, and the compatibility condition can be formulated with respect to these traces.

In this paper, to prove Theorem 1.3, we prove the existence of $\mathcal{R}$-bounded solution operators associated with problem (1-5). Namely, we prove 
Theorem 1.4 (Existence of $\mathcal{R}$-bounded solution operators). Let $1<q<\infty$. Assume that $\Omega$ is a uniform $C^{4}$-domain in $\mathbb{R}^{N}$. Let $\mathbb{G}_{q}(\Omega)$ and $\mathcal{X}_{q}(\Omega)$ be defined as in (1-7). Then, there exist a number $\vartheta>\pi / 2$, a positive number $\lambda_{0}$, and an operator family $\mathcal{B}_{i}(\lambda)(i=1,2)$ with

$$
\mathcal{B}_{1}(\lambda) \in \mathcal{C}\left(\Sigma_{\vartheta, \lambda_{0}}, \mathcal{L}\left(\mathcal{X}_{q}(\Omega), H_{q}^{4}(\Omega)\right)\right), \quad \mathcal{B}_{2}(\lambda) \in \mathcal{C}\left(\Sigma_{\vartheta, \lambda_{0}}, \mathcal{L}\left(\mathcal{X}_{q}(\Omega), H_{q}^{2}(\Omega)\right)\right)
$$

such that problem (1-5) admits a unique solution $U=\mathcal{B}(\lambda) \mathcal{H}_{\lambda}(F, G)$ with $\mathcal{B}(\lambda)=\left(\mathcal{B}_{1}(\lambda), \lambda \mathcal{B}_{1}(\lambda), \mathcal{B}_{2}(\lambda)\right)^{\top}$ for any $(F, G) \in \mathbb{G}_{q}(\Omega)$ and $\lambda \in \Sigma_{\vartheta, \lambda_{0}}$, where $\mathcal{H}_{\lambda}(F, G)=\left(f_{1}, f_{2}, G, \lambda^{1 / 2} G, \lambda g_{1}\right)$ for $F=\left(0, f_{1}, f_{2}\right)^{\top}$ and $G=\left(g_{1}, g_{2}, g_{3}\right)^{\top}$, and there hold the estimates:

$$
\begin{aligned}
& \mathcal{R}_{\mathcal{L}\left(\mathcal{X}_{q}(\Omega), H_{q}^{4-j}(\Omega)\right)}\left(\left\{\left(\tau \partial_{\tau}\right)^{s}\left(\lambda^{j / 2} \mathcal{B}_{1}(\lambda)\right) \mid \lambda \in \Sigma_{\vartheta, \lambda_{0}}\right\}\right) \leq C \quad(s=0,1, \quad j=0,1,2,3,4), \\
& \mathcal{R}_{\mathcal{L}\left(\mathcal{X}_{q}(\Omega), H_{q}^{2-j}(\Omega)\right)}\left(\left\{\left(\tau \partial_{\tau}\right)^{s}\left(\lambda^{j / 2} \mathcal{B}_{2}(\lambda)\right) \mid \lambda \in \Sigma_{\vartheta, \lambda_{0}}\right\}\right) \leq C \quad(s=0,1, \quad j=0,1,2) .
\end{aligned}
$$

\section{Analysis in the Whole space}

The purpose of this section is to prove the existence of an $\mathcal{R}$-bounded solution operator associated with the resolvent problem:

$$
\lambda U-A(D) U=F \quad \text { in } \mathbb{R}^{N}
$$

with $F=\left(0, f_{1}, f_{2}\right)^{\top}$. One main tool for the proof is the following lemma due to Denk and Schnaubelt [7, Lemma 2.1] and Enomoto and Shibata [9, Theorem 3.3].

Lemma 2.1. Let $1<q<\infty$ and let $\Lambda$ be a set in $\mathbb{C}$. Let $m=m(\lambda, \xi)$ be a function defined on $\Lambda \times\left(\mathbb{R}^{N} \backslash\{0\}\right)$ which is infinitely differentiable with respect to $\xi \in \mathbb{R}^{N} \backslash\{0\}$ for each $\lambda \in \Lambda$. Assume that for any multi-index $\alpha \in \mathbb{N}_{0}^{N}$ there exists a constant $C_{\alpha}$ depending on $\alpha$ and $\Lambda$ such that

$$
\left|\partial_{\xi}^{\alpha} m(\lambda, \xi)\right| \leq C_{\alpha}|\xi|^{-|\alpha|}
$$

for any $(\lambda, \xi) \in \Lambda \times\left(\mathbb{R}^{N} \backslash\{0\}\right)$. Let $K_{\lambda}$ be an operator defined by $K_{\lambda} f=\mathscr{F}_{\xi}^{-1}[m(\lambda, \xi) \mathscr{F} f(\xi)]$. Then, the family of operators $\left\{K_{\lambda} \mid \lambda \in \Lambda\right\}$ is $\mathcal{R}$-bounded on $\mathcal{L}\left(L_{q}\left(\mathbb{R}^{N}\right)\right)$ and

$$
\mathcal{R}_{\mathcal{L}\left(L_{q}\left(\mathbb{R}^{N}\right)\right)}\left(\left\{K_{\lambda} \mid \lambda \in \Lambda\right\}\right) \leq C_{q, N} \max _{|\alpha| \leq N+1} C_{\alpha}
$$

with some constant $C_{q, N}$ depending only on $q$ and $N$.

The symbol of the operator matrix $A(D)$ is given by

$$
A(\xi):=\left(\begin{array}{ccc}
0 & 1 & 0 \\
-|\xi|^{4} & 0 & |\xi|^{2} \\
0 & -|\xi|^{2} & -|\xi|^{2}
\end{array}\right),
$$

i.e., we have $A(D)=\mathscr{F}^{-1} A(\cdot) \mathscr{F}$. Thus, by the Fourier transform equation $(2-1)$ is transformed to

$$
(\lambda I-A(\xi)) \hat{U}(\xi)=\hat{F}(\xi)
$$

with $\hat{U}=(\mathscr{F} u, \mathscr{F} v, \mathscr{F} \theta)^{\top}$ and $\hat{F}=\left(0, \mathscr{F} f_{1}, \mathscr{F} f_{2}\right)^{\top}$. The analysis of the inverse matrix $(\lambda I-A(\xi))^{-1}$ was essentially done in [24]. As we need some variants of the results in [24], we summarize the main properties of $(\lambda I-A(\xi))^{-1}$ and give a short indication of the proofs.

In the following, define $\gamma_{1}, \gamma_{2}, \gamma_{3}$ by the equality

$$
p(t):=t^{3}+t^{2}+2 t+1=\left(t+\gamma_{1}\right)\left(t+\gamma_{2}\right)\left(t+\gamma_{3}\right)
$$

with $\gamma_{1} \in \mathbb{R}, \gamma_{2}=\bar{\gamma}_{3}$ and $\operatorname{Im} \gamma_{2}>0$. Then $\gamma_{1} \in(0,1), \operatorname{Re} \gamma_{2}=\operatorname{Re} \gamma_{3} \in\left(0, \frac{1}{2}\right)$, and $\operatorname{det}(\lambda-A(\xi))=$ $\prod_{j=1}^{3}\left(\lambda+\gamma_{j}|\xi|^{2}\right)$ (see [24, Lemma 2.3]). Let $\vartheta_{0}>\pi / 2$ and $\vartheta_{1}>\pi / 2$ be chosen in such a way that

$$
\lambda \gamma_{i}^{-1} \in \Sigma_{\vartheta_{1}} \quad(i=1,2,3) \text { for } \lambda \in \Sigma_{\vartheta_{0}} .
$$


Then the inequality

$$
\left.\left|\lambda \gamma_{i}^{-1}+\right| \xi\right|^{2} \mid \geq c\left(|\lambda|+|\xi|^{2}\right)
$$

holds for any $\lambda \in \Sigma_{\vartheta_{0}}$ and $\xi \in \mathbb{R}^{N}$ with some positive constant $c$.

It was shown in [24, Section 2] that for all $\lambda \in \Sigma_{\vartheta_{0}}$ we have

$$
(\lambda I-A(\xi))^{-1}=\frac{1}{\operatorname{det}(\lambda I-A(\xi))}\left(\begin{array}{ccc}
\lambda\left(\lambda+|\xi|^{2}\right)+|\xi|^{4} & \lambda+|\xi|^{2} & |\xi|^{2} \\
-\left(\lambda+|\xi|^{2}\right)|\xi|^{4} & \lambda\left(\lambda+|\xi|^{2}\right) & \lambda|\xi|^{2} \\
|\xi|^{6} & -\lambda|\xi|^{2} & \lambda^{2}+|\xi|^{4}
\end{array}\right)
$$

Since $\gamma_{1} \gamma_{2} \gamma_{3}=1$ as follows from Vieta's formula, we have $\operatorname{det}(\lambda I-A(\xi))=\prod_{i=1}^{3}\left(\lambda \gamma_{i}^{-1}+|\xi|^{2}\right)$, and then a solution $U=(u, \lambda u, \theta)^{\top}$ of problem $(2-1)$ is given by $U=\mathscr{F}^{-1}\left((\lambda I-A(\xi))^{-1} \mathscr{F} f(\xi)\right)$, i.e.,

$$
\begin{aligned}
& u(x)=\mathscr{F}^{-1}\left[\frac{\lambda+|\xi|^{2}}{\prod_{i=1}^{3}\left(\lambda \gamma_{i}^{-1}+|\xi|^{2}\right)} \mathscr{F} f_{1}(\xi)\right]+\mathscr{F}^{-1}\left[\frac{|\xi|^{2}}{\prod_{i=1}^{3}\left(\lambda \gamma_{i}^{-1}+|\xi|^{2}\right)} \mathscr{F} f_{2}(\xi)\right], \\
& \theta(x)=-\mathscr{F}^{-1}\left[\frac{\lambda|\xi|^{2}}{\prod_{i=1}^{3}\left(\lambda \gamma_{i}^{-1}+|\xi|^{2}\right)} \mathscr{F} f_{1}(\xi)\right]+\mathscr{F}^{-1}\left[\frac{\lambda^{2}+|\xi|^{4}}{\prod_{i=1}^{3}\left(\lambda \gamma_{i}^{-1}+|\xi|^{2}\right)} \mathscr{F} f_{2}(\xi)\right] .
\end{aligned}
$$

Let the operator $\mathcal{S}_{0}(\lambda)$ acting on $f$ be defined by

$$
\mathcal{S}_{0}(\lambda) f:=\mathscr{F}^{-1}\left[\left(\prod_{i=1}^{3}\left(\lambda \gamma_{i}+|\xi|^{2}\right)\right)^{-1} \mathscr{F} f(\xi)\right] .
$$

By Lemma 2.1 and (2-7),

$$
\mathcal{R}_{\mathcal{L}\left(L_{q}\left(\mathbb{R}^{N}\right)\right)}\left(\left\{\left(\tau \partial_{\tau}\right)^{s}\left(\lambda^{j / 2} \partial_{x}^{\alpha} \mathcal{S}_{0}(\lambda)\right) \mid \lambda \in \Sigma_{\vartheta_{0}}\right\}\right) \leq C_{j, \alpha} \quad(s=0,1)
$$

for $j \in \mathbb{N}_{0}$ and $\alpha \in \mathbb{N}_{0}^{N}$ with $j+|\alpha|=6$. Moreover, by (2-8) $u=\mathcal{S}_{1}(\lambda) F$ and $\theta=\mathcal{S}_{2}(\lambda) F$ for $F=\left(f_{1}, f_{2}\right)^{\top}$ with

$$
\begin{aligned}
& \mathcal{S}_{1}(\lambda) F:=(\lambda-\Delta) \mathcal{S}_{0}(\lambda) f_{1}-\Delta S_{0}(\lambda) f_{2} \\
& \mathcal{S}_{2}(\lambda) F:=\lambda \Delta \mathcal{S}_{0}(\lambda) f_{1}+\left(\lambda^{2}+\Delta^{2}\right) \mathcal{S}_{0}(\lambda) f_{2}
\end{aligned}
$$

At this point, we introduce some fundamental properties of $\mathcal{R}$-bounded operators and Bourgain's results concerning Fourier multiplier theorems with scalar multiplier.

Proposition 2.2. a) Let $X$ and $Y$ be Banach spaces, and let $\mathcal{T}$ and $\mathcal{S}$ be $\mathcal{R}$-bounded families in $\mathcal{L}(X, Y)$. Then, $\mathcal{T}+\mathcal{S}=\{T+S \mid T \in \mathcal{T}, S \in \mathcal{S}\}$ is also an $\mathcal{R}$-bounded family in $\mathcal{L}(X, Y)$ and

$$
\mathcal{R}_{\mathcal{L}(X, Y)}(\mathcal{T}+\mathcal{S}) \leq \mathcal{R}_{\mathcal{L}(X, Y)}(\mathcal{T})+\mathcal{R}_{\mathcal{L}(X, Y)}(\mathcal{S})
$$

b) Let $X, Y$ and $Z$ be Banach spaces, and let $\mathcal{T}$ and $\mathcal{S}$ be $\mathcal{R}$-bounded families in $\mathcal{L}(X, Y)$ and $\mathcal{L}(Y, Z)$, respectively. Then, $\mathcal{S} \mathcal{T}=\{S T \mid T \in \mathcal{T}, S \in \mathcal{S}\}$ also an $\mathcal{R}$-bounded family in $\mathcal{L}(X, Z)$ and

$$
\mathcal{R}_{\mathcal{L}(X, Z)}(\mathcal{S T}) \leq \mathcal{R}_{\mathcal{L}(X, Y)}(\mathcal{T}) \mathcal{R}_{\mathcal{L}(Y, Z)}(\mathcal{S}) .
$$

c) Let $1<p, q<\infty$ and let $D$ be a domain in $\mathbb{R}^{N}$. Let $m=m(\lambda)$ be a bounded function defined on a subset $\Lambda$ in $\mathbb{C}$ and let $M_{m}(\lambda)$ be a map defined by $M_{m}(\lambda) f=m(\lambda) f$ for any $f \in L_{q}(D)$. Then, $\mathcal{R}_{\mathcal{L}\left(L_{q}(D)\right)}\left(\left\{M_{m}(\lambda) \mid \lambda \in \Lambda\right\}\right) \leq C_{N, q, D}\|m\|_{L_{\infty}(\Lambda)}$.

d) Let $n=n(\tau)$ be a $C^{1}$-function defined on $\mathbb{R} \backslash\{0\}$ that satisfies the conditions $|n(\tau)| \leq \gamma$ and $\left|\tau n^{\prime}(\tau)\right| \leq \gamma$ with some constant $c>0$ for any $\tau \in \mathbb{R} \backslash\{0\}$. Let $T_{n}$ be the operator-valued Fourier multiplier defined by $T_{n} f=\mathscr{F}^{-1}(n \mathscr{F}[f])$ for any $f$ with $\mathscr{F}[f] \in \mathscr{D}\left(\mathbb{R}, L_{q}(D)\right)$. Then, $T_{n}$ is extended to a bounded linear operator from $L_{p}\left(\mathbb{R}, L_{q}(D)\right)$ into itself. Moreover, denoting this extension also by $T_{n}$, we have

$$
\left\|T_{n}\right\|_{\mathcal{L}\left(L_{p}\left(\mathbb{R}, L_{q}(D)\right)\right)} \leq C_{p, q, D} \gamma .
$$

Here, $\mathscr{D}\left(\mathbb{R}, L_{q}(D)\right)$ denotes the set of all $L_{q}(D)$-valued $C^{\infty}$-functions on $\mathbb{R}$ with compact support. 
Proof. The assertions a) and b) follow from [3, p.28, Proposition 3.4], and the assertions c) and d) follow from [3, p.27, Remarks 3.2] (see also Bourgain [1]).

Since

$$
\left|\partial_{\xi}^{\alpha}\left(\tau \partial_{\tau}\right)^{s}\left(\frac{\lambda^{j / 2}(i \xi)^{\beta}}{\prod_{i=1}^{3}\left(\lambda \gamma_{i}^{-1}+|\xi|^{2}\right)}\right)\right| \leq C_{\alpha}|\xi|^{-|\alpha|}
$$

for any $s \in\{0,1\}, j \in \mathbb{N}_{0}$ and $\beta \in \mathbb{N}_{0}^{N}$ with $j+|\beta|=6$ and $(\lambda, \xi) \in \Sigma_{\vartheta_{0}} \times\left(\mathbb{R}^{N} \backslash\{0\}\right)$ as follows from (2-7), by Lemma 2.1 and Proposition $2.2 \mathrm{a})$,

$$
\begin{aligned}
& \mathcal{R}_{\mathcal{L}\left(L_{q}\left(\mathbb{R}^{N}\right)\right)}\left(\left\{\left(\tau \partial_{\tau}\right)^{s}\left(\lambda^{j / 2} \partial_{x}^{\alpha}(\lambda-\Delta) \mathcal{S}_{0}(\lambda)\right) \mid \lambda \in \Sigma_{\vartheta_{0}}\right\}\right) \leq \gamma_{0} \quad(s=0,1, \quad j=0,1,2,3,4 j+|\alpha|=4), \\
& \mathcal{R}_{\mathcal{L}\left(L_{q}\left(\mathbb{R}^{N}\right)\right)}\left(\left\{\left(\tau \partial_{\tau}\right)^{s}\left(\lambda^{j / 2} \partial_{x}^{\alpha} \Delta \mathcal{S}_{0}(\lambda)\right) \mid \lambda \in \Sigma_{\vartheta_{0}}\right\}\right) \leq \gamma_{0} \quad(s=0,1, \quad j=0,1,2,3,4, j+|\alpha|=4), \\
& \mathcal{R}_{\mathcal{L}\left(L_{q}\left(\mathbb{R}^{N}\right)\right)}\left(\left\{\left(\tau \partial_{\tau}\right)^{s}\left(\lambda^{j / 2} \partial_{x}^{\alpha}\left(\lambda \Delta \mathcal{S}_{0}(\lambda)\right)\right) \mid \lambda \in \Sigma_{\vartheta_{0}}\right\}\right) \leq \gamma_{0} \quad(s=0,1, \quad j=0,1,2, j+|\alpha|=2), \\
& \mathcal{R}_{\mathcal{L}\left(L_{q}\left(\mathbb{R}^{N}\right)\right)}\left(\left\{\left(\tau \partial_{\tau}\right)^{s}\left(\lambda^{j / 2} \partial_{x}^{\alpha}\left(\lambda^{2}+\Delta^{2}\right) \mathcal{S}_{0}(\lambda)\right) \mid \lambda \in \Sigma_{\vartheta_{0}}\right\}\right) \leq \gamma_{0} \quad(s=0,1, \quad j=0,1,2, j+|\alpha|=2)
\end{aligned}
$$

for some constant $\gamma_{0}>0$. Combined with Proposition $2.2 \mathrm{c}$ ), this yields the following result.

Theorem 2.3. Let $1<q<\infty$ and $\lambda_{0}>0$. Let $\vartheta_{0}$ be the number given in (2-6). Then, there exist operator families $\mathcal{S}_{i}(\lambda)(i=1,2)$ with

$$
\mathcal{S}_{1} \in \mathcal{C}\left(\Sigma_{\vartheta_{0}, \lambda_{0}}, \mathcal{L}\left(L_{q}\left(\mathbb{R}^{N}\right)^{2}, H_{q}^{4}\left(\mathbb{R}^{N}\right)\right)\right), \quad \mathcal{S}_{2} \in \mathcal{C}\left(\Sigma_{\vartheta_{0}, \lambda_{0}}, \mathcal{L}\left(L_{q}\left(\mathbb{R}^{N}\right)^{2}, H_{q}^{2}\left(\mathbb{R}^{N}\right)\right)\right)
$$

such that problem (2-1) admits a unique solution $U=\left(\mathcal{S}_{1}(\lambda) F^{\prime}, \lambda \mathcal{S}_{1}(\lambda) F^{\prime}, \mathcal{S}_{2}(\lambda) F^{\prime}\right)^{\top}$ for any $\lambda \in \Sigma_{\vartheta_{0}, \lambda_{0}}$ and $F=\left(0, f_{1}, f_{2}\right)^{\top}$ with $F^{\prime}=\left(f_{1}, f_{2}\right)^{\top} \in L_{q}\left(\mathbb{R}^{N}\right)^{2}$, and there hold the estimates:

$$
\begin{aligned}
& \mathcal{R}_{\mathcal{L}\left(L_{q}\left(\mathbb{R}^{N}\right)^{2}, H_{q}^{4-j}\left(\mathbb{R}^{N}\right)\right)}\left(\left\{\left(\tau \partial_{\tau}\right)^{s}\left(\lambda^{j / 2} \mathcal{S}_{1}(\lambda)\right) \mid \lambda \in \Sigma_{\vartheta_{0}, \lambda_{0}}\right\}\right) \leq C_{\lambda_{0}} \gamma_{0} \quad(s=0,1, \quad j=0,1,2,3,4), \\
& \mathcal{R}_{\mathcal{L}\left(L_{q}\left(\mathbb{R}^{N}\right)^{2}, H_{q}^{2-j}\left(\mathbb{R}^{N}\right)\right)}\left(\left\{\left(\tau \partial_{\tau}\right)^{s}\left(\lambda^{j / 2} \mathcal{S}_{2}(\lambda)\right) \mid \lambda \in \Sigma_{\vartheta_{0}, \lambda_{0}}\right\}\right) \leq C_{\lambda_{0}} \gamma_{0} \quad(s=0,1, \quad j=0,1,2),
\end{aligned}
$$

with some constant $C_{\lambda_{0}}>0$.

\section{Solution operators in the half-SpaCe}

Let $\mathbb{R}_{+}^{N}=\left\{x=\left(x_{1}, \ldots, x_{N}\right) \in \mathbb{R}^{N} \mid x_{N}>0\right\}$ and $\mathbb{R}_{0}^{N}=\left\{x=\left(x_{1}, \ldots, x_{N}\right) \in \mathbb{R}^{N} \mid x_{N}=0\right\}$. The purpose of this section is to prove the existence of $\mathcal{R}$-bounded solution operators of the generalized resolvent problem:

$$
\lambda U-A(D) U=F \text { in } \mathbb{R}_{+}^{N}, \quad B(D) U=G \text { on } \mathbb{R}_{0}^{N}
$$

in the half-space $\mathbb{R}_{+}^{N}$ with $U=(u, \lambda u, \theta)^{\top}, F=\left(0, f_{1}, f_{2}\right)^{\top}$ and $G=\left(g_{1}, g_{2}, g_{3}\right)^{\top}$. The boundary condition in (3-1) is represented componentwise by

$$
\begin{aligned}
\Delta u-(1-\beta) \Delta^{\prime} u+\theta & =g_{1} & & \text { on } \mathbb{R}_{0}^{N} \\
\partial_{N}\left(\Delta u+(1-\beta) \Delta^{\prime} u\right) & =g_{2} & & \text { on } \mathbb{R}_{0}^{N} \\
\partial_{N} \theta & =g_{3} & & \text { on } \mathbb{R}_{0}^{N}
\end{aligned}
$$

Here, $\Delta^{\prime}=\sum_{j=1}^{N-1} \partial_{j}^{2}$. Then, the main result of this section is

Theorem 3.1. Let $1<q<\infty$ and $\lambda_{0}>0$. Then, there exist a number $\vartheta>\pi / 2$ and operator families $\mathcal{T}_{i}(\lambda)(i=1,2)$ with

$$
\mathcal{T}_{1} \in \mathcal{C}\left(\Sigma_{\vartheta}, \mathcal{L}\left(\mathcal{X}_{q}\left(\mathbb{R}_{+}^{N}\right), H_{q}^{4}\left(\mathbb{R}_{+}^{N}\right)\right)\right), \quad \mathcal{T}_{2} \in \mathcal{C}\left(\Sigma_{\vartheta}, \mathcal{L}\left(\mathcal{X}_{q}\left(\mathbb{R}_{+}^{N}\right), H_{q}^{2}\left(\mathbb{R}_{+}^{N}\right)\right)\right)
$$

such that problem (3-1) admits a unique solution

$$
U=\left(\mathcal{T}_{1}(\lambda) H_{\lambda}(F, G), \lambda \mathcal{T}_{1}(\lambda) H_{\lambda}(F, G), \mathcal{T}_{2}(\lambda) H_{\lambda}(F, G)\right)^{\top}
$$


for any $(F, G) \in \mathbb{G}_{q}\left(\mathbb{R}_{+}^{N}\right)$ and $\lambda \in \Sigma_{\vartheta}$, and there hold the estimates:

$$
\begin{aligned}
& \mathcal{R}_{\mathcal{L}\left(\mathcal{X}_{q}\left(\mathbb{R}_{+}^{N}\right), H_{q}^{4-j}\left(\mathbb{R}_{+}^{N}\right)\right)}\left(\left\{\left(\tau \partial_{\tau}\right)^{s}\left(\lambda^{j / 2} \mathcal{T}_{1}(\lambda)\right) \mid \lambda \in \Sigma_{\vartheta, \lambda_{0}}\right\}\right) \leq C_{N, q, \lambda_{0}} \quad(s=0,1, \quad j=0,1,2,3,4), \\
& \mathcal{R}_{\mathcal{L}\left(\mathcal{X}_{q}\left(\mathbb{R}_{+}^{N}\right), H_{q}^{2-j}\left(\mathbb{R}_{+}^{N}\right)\right)}\left(\left\{\left(\tau \partial_{\tau}\right)^{s}\left(\lambda^{j / 2} \mathcal{T}_{2}(\lambda)\right) \mid \lambda \in \Sigma_{\vartheta, \lambda_{0}}\right\}\right) \leq C_{N, q, \lambda_{0}} \quad(s=0,1, \quad j=0,1,2) .
\end{aligned}
$$

In what follows, we prove Theorem 3.1. Let $\iota_{h}$ be the Lions extension operator of the form:

$$
\left[\iota_{h} f\right](x):= \begin{cases}f\left(x^{\prime}, x_{N}\right) & \left(x_{N}>0\right), \\ \sum_{j=1}^{6} a_{j} f\left(x^{\prime},-j x_{N}\right) & \left(x_{N}<0\right),\end{cases}
$$

for any given $f$ on $\mathbb{R}_{+}^{N}$, where $x^{\prime}=\left(x_{1}, \ldots, x_{N-1}\right)$, and $a_{j}$ are real numbers satisfying the relations:

$$
\sum_{j=1}^{6}(-j)^{k} a_{j}=1 \quad \text { for } k=-1,0,1, \ldots, 4 .
$$

Let $\mathcal{S}(\lambda)=\left(\mathcal{S}_{1}(\lambda), \mathcal{S}_{2}(\lambda)\right)^{\top}$ be the solution operator given in Theorem 2.3, and let the operator $\mathcal{S}_{+}(\lambda)=$ $\left(\mathcal{S}_{+1}(\lambda), \mathcal{S}_{+2}(\lambda)\right)^{\top}$ acting on $F^{\prime}=\left(f_{1}, f_{2}\right) \in L_{q}\left(\mathbb{R}_{+}^{N}\right)^{2}$ be defined by

$$
\mathcal{S}_{+i}(\lambda) F^{\prime}=\mathcal{S}_{i}(\lambda)\left(\iota_{h} F^{\prime}\right) \quad(i=1,2) .
$$

Obviously,

$$
V=\left(\mathcal{S}_{+1}(\lambda)\left(\iota_{h} F^{\prime}\right), \lambda \mathcal{S}_{+1}(\lambda)\left(\iota_{h} F^{\prime}\right), \mathcal{S}_{+2}(\lambda)\left(\iota_{h} F^{\prime}\right)\right)^{\top}
$$

satisfies the equation: $\lambda V-A(D) V=F$ in $\mathbb{R}_{+}^{N}$ with $F=\left(0, f_{1}, f_{2}\right)^{\top}$ and the estimate:

$$
\begin{aligned}
& \mathcal{R}_{\mathcal{L}\left(L_{q}\left(\mathbb{R}_{+}^{N}\right)^{2}, H_{q}^{4-j}\left(\mathbb{R}_{+}^{N}\right)\right)}\left(\left\{\left(\tau \partial_{\tau}\right)^{s}\left(\lambda^{j / 2} \mathcal{S}_{+1}(\lambda)\right) \mid \lambda \in \Sigma_{\vartheta_{0}, \lambda_{0}}\right\}\right) \leq C_{\lambda_{0}} \quad(s=0,1, \quad j=0,1,2,3,4), \\
& \mathcal{R}_{\mathcal{L}\left(L_{q}\left(\mathbb{R}_{+}^{N}\right)^{2}, H_{q}^{2-j}\left(\mathbb{R}_{+}^{N}\right)\right)}\left(\left\{\left(\tau \partial_{\tau}\right)^{s}\left(\lambda^{j / 2} \mathcal{S}_{+2}(\lambda)\right) \mid \lambda \in \Sigma_{\vartheta_{0}, \lambda_{0}}\right\}\right) \leq C_{\lambda_{0}} \quad(s=0,1, \quad j=0,1,2) .
\end{aligned}
$$

Setting $U=V+W$ yields that $W$ should solve the equations (3-1) replacing $F$ and $G$ by 0 and $G-B(D) V$. Since the second component of $W$ coincides with $\lambda$ times the first component, in what follows, it suffices to consider the equations:

$$
\lambda^{2} u+\Delta^{2} u+\Delta \theta=0, \quad \lambda \theta-\Delta \theta-\lambda \Delta u=0 \quad \text { in } \mathbb{R}_{+}^{N}
$$

with non-homogeneous boundary condition (3-2).

Applying partial Fourier transform

$$
\mathscr{F}^{\prime}[u]\left(\xi^{\prime}, x_{N}\right):=\int_{\mathbb{R}^{N-1}} u\left(x^{\prime}, x_{N}\right) e^{-i x^{\prime} \xi^{\prime}} d x^{\prime},
$$

where $\xi^{\prime}=\left(\xi_{1}, \ldots, \xi_{N-1}\right)$, to (3-6) and (3-2) yields an ordinary differential equation system in $x_{N}>0$ :

$$
\begin{aligned}
\lambda^{2} w+\left(\partial_{N}^{2}-\left|\xi^{\prime}\right|^{2}\right)^{2} w+\left(\partial_{N}^{2}-\left|\xi^{\prime}\right|^{2}\right) \tau=0 & \left(x_{N}>0\right), \\
\lambda \tau-\left(\partial_{N}^{2}-\left|\xi^{\prime}\right|^{2}\right) \tau-\lambda\left(\partial_{N}^{2}-\left|\xi^{\prime}\right|^{2}\right) w=0 & \left(x_{N}>0\right)
\end{aligned}
$$

with initial conditions

$$
\begin{aligned}
\left(\partial_{N}^{2}-\left|\xi^{\prime}\right|^{2}\right) w(0)+(1-\beta)\left|\xi^{\prime}\right|^{2} w(0)+\tau(0) & =\mathscr{F}^{\prime}\left[g_{1}\right]\left(\xi^{\prime}, 0\right), \\
\partial_{N}\left(\left(\partial_{N}^{2}-\left|\xi^{\prime}\right|^{2}\right) w(0)-(1-\beta)\left|\xi^{\prime}\right|^{2} w(0)\right) & =\mathscr{F}^{\prime}\left[g_{2}\right]\left(\xi^{\prime}, 0\right), \\
\partial_{N} \tau(0) & =\mathscr{F}^{\prime}\left[g_{3}\right]\left(\xi^{\prime}, 0\right) .
\end{aligned}
$$

Here we have set $w\left(\xi^{\prime}, x_{N}\right)=\left(\mathscr{F}^{\prime} u\right)\left(\xi^{\prime}, x_{N}\right)$ and $\tau\left(\xi^{\prime}, x_{N}\right)=\left(\mathscr{F}^{\prime} \theta\right)\left(\xi^{\prime}, x_{N}\right)$. 
We find solutions $w$ and $\tau$ of (3-7)-(3-8). For this, we use the representation formula of $w$ and $\tau$ which was derived in [24, Eq. (3.15)]. There it was shown that every stable solution of (3-7) has the form

$$
\begin{aligned}
& w\left(\xi^{\prime}, x_{N}, \lambda\right)=\sum_{i=1}^{3} P_{i} \exp \left(-A_{i}\left(\xi^{\prime}, \lambda\right) x_{N}\right) \\
& \tau\left(\xi^{\prime}, x_{N}, \lambda\right)=-\lambda \sum_{i=1}^{3}\left(\gamma_{i}^{2}+2\right) P_{i} \exp \left(-A_{i}\left(\xi^{\prime}, \lambda\right) x_{N}\right) .
\end{aligned}
$$

Here $\gamma_{1}, \gamma_{2}, \gamma_{3}$ are given by (2-5). The numbers $A_{i}$ appearing in (3-9) are defined by

$$
A_{k}\left(\xi^{\prime}, \lambda\right)=\sqrt{\lambda \gamma_{i}^{-1}+\left|\xi^{\prime}\right|^{2}} \quad(k=1,2,3),
$$

and $P_{1}, P_{2}, P_{3}$ are constants which will determined later by the boundary conditions. Inserting (3-9) into the boundary conditions (3-8), we get a linear equation system for the coefficients $P_{i}$ :

$$
\begin{aligned}
\sum_{i=1}^{3}\left(A_{i}^{2}-\beta\left|\xi^{\prime}\right|^{2}-\lambda\left(\gamma_{i}^{2}+2\right)\right) P_{i} & =\mathscr{F}^{\prime}\left[g_{1}\right]\left(\xi^{\prime}, 0\right), \\
\sum_{i=1}^{3}\left(-A_{i}^{3}+A_{i}(2-\beta)\left|\xi^{\prime}\right|^{2}\right) P_{i} & =\mathscr{F}^{\prime}\left[g_{2}\right]\left(\xi^{\prime}, 0\right), \\
\sum_{i=1}^{3} \lambda A_{i}\left(\gamma_{i}^{2}+2\right) P_{i} & =\mathscr{F}^{\prime}\left[g_{3}\right]\left(\xi^{\prime}, 0\right) .
\end{aligned}
$$

Noting $A_{i}^{2}=\lambda \gamma_{i}^{-1}+\left|\xi^{\prime}\right|^{2}$ and $\gamma_{i}^{-1}-\gamma_{i}^{2}-2=\frac{1}{\gamma_{i}}\left(1-\gamma_{i}^{3}-2 \gamma_{i}\right)=-\gamma_{i}$ by $p\left(-\gamma_{i}\right)=0$, the linear equations above are re-written of the form:

$$
\Delta\left(\xi^{\prime}, \lambda\right)\left(P_{1}, P_{2}, P_{3}\right)^{\top}=\left(\mathscr{F}^{\prime}\left[g_{1}\right]\left(\xi^{\prime}, 0\right), \mathscr{F}^{\prime}\left[g_{2}\right]\left(\xi^{\prime}, 0\right), \mathscr{F}^{\prime}\left[g_{3}\right]\left(\xi^{\prime}, 0\right)\right)^{\top}
$$

with

$$
\Delta\left(\xi^{\prime}, \lambda\right):=\left(\begin{array}{ccc}
-\gamma_{1} \lambda+\zeta & -\gamma_{2} \lambda+\zeta & -\gamma_{3} \lambda+\zeta \\
A_{1}\left(-\frac{\lambda}{\gamma_{1}}+\zeta\right) & A_{2}\left(-\frac{\lambda}{\gamma_{2}}+\zeta\right) & A_{3}\left(-\frac{\lambda}{\gamma_{3}}+\zeta\right) \\
\lambda A_{1}\left(\gamma_{1}^{2}+2\right) & \lambda A_{2}\left(\gamma_{2}^{2}+2\right) & \lambda A_{3}\left(\gamma_{3}^{2}+2\right)
\end{array}\right)
$$

Here $\zeta:=(1-\beta)\left|\xi^{\prime}\right|^{2}$. The matrix $\Delta\left(\xi^{\prime}, \lambda\right)$ is called the Lopatinskiu matrix of (3-6), (3-2).

It is the most important of this paper to analyze the inverse matrix of the Lopatinskiu matrix. For this purpose, we introduce some classes of multipliers.

Definition 3.2. Let $V$ be a domain in $\mathbb{C}$, let $\Xi \subset\left(\mathbb{R}^{N-1} \backslash\{0\}\right) \times V$, and let $m: \Xi \rightarrow \mathbb{C},\left(\xi^{\prime}, \lambda\right) \mapsto m\left(\xi^{\prime}, \lambda\right)$ be $C^{1}$ with respect to $\tau$ (where $\lambda=\gamma+i \tau$ ) and $C^{\infty}$ with respect to $\xi^{\prime}$.

(1) $m\left(\xi^{\prime}, \lambda\right)$ is called a multiplier of order $s$ with type 1 on $\Xi$ if there hold the estimates:

$$
\left|\partial_{\xi^{\prime}}^{\kappa^{\prime}} m\left(\xi^{\prime}, \lambda\right)\right| \leq C_{\kappa^{\prime}}\left(|\lambda|^{1 / 2}+\left|\xi^{\prime}\right|\right)^{s-\left|\kappa^{\prime}\right|}, \quad\left|\partial_{\xi^{\prime}}^{\kappa^{\prime}}\left(\tau \partial_{\tau} m\left(\xi^{\prime}, \lambda\right)\right)\right| \leq C_{\kappa^{\prime}}\left(|\lambda|^{1 / 2}+\left|\xi^{\prime}\right|\right)^{s-\left|\kappa^{\prime}\right|}
$$

for any multi-index $\kappa^{\prime} \in \mathbb{N}_{0}^{N-1}$ and $\left(\xi^{\prime}, \lambda\right) \in \Xi$ with some constant $C_{\kappa^{\prime}}$ depending solely on $\kappa^{\prime}$ and $\Xi$.

(2) $m\left(\xi^{\prime}, \lambda\right)$ is called a multiplier of order $s$ with type 2 on $\Xi$ if there hold the estimates:

$$
\left|\partial_{\xi^{\prime}}^{\kappa^{\prime}} m\left(\xi^{\prime}, \lambda\right)\right| \leq C_{\kappa^{\prime}}\left(|\lambda|^{1 / 2}+\left|\xi^{\prime}\right|\right)^{s}\left|\xi^{\prime}\right|^{-\left|\kappa^{\prime}\right|}, \quad\left|\partial_{\xi^{\prime}}^{\kappa^{\prime}}\left(\tau \partial_{\tau} m\left(\xi^{\prime}, \lambda\right)\right)\right| \leq C_{\kappa^{\prime}}\left(|\lambda|^{1 / 2}+\left|\xi^{\prime}\right|\right)^{s}\left|\xi^{\prime}\right|^{-\left|\kappa^{\prime}\right|} .
$$

for any multi-index $\kappa^{\prime} \in \mathbb{N}_{0}^{N-1}$ and $\left(\xi^{\prime}, \lambda\right) \in \Xi$ with some constant $C_{\kappa^{\prime}}$ depending solely on $\kappa^{\prime}$ and $\Xi$

Let $\mathbb{M}_{s, i}(\Xi)$ be the set of all multipliers of order $s$ with type $i$ on $\Xi(i=1,2)$. In the standard case $\Xi=\left(\mathbb{R}^{N-1} \backslash\{0\}\right) \times V$, we write $\mathbb{M}_{s, i}(V)$ instead of $\mathbb{M}_{s, i}(\Xi)$. 
Obviously, $\mathbb{M}_{s, i}(\Xi)$ are complex vector spaces. Moreover, the following lemma follows from the inequality $\left(|\lambda|^{1 / 2}+\left|\xi^{\prime}\right|\right)^{-\left|\alpha^{\prime}\right|} \leq\left|\xi^{\prime}\right|^{-\left|\alpha^{\prime}\right|}$ and the Leibniz rule immediately.

Lemma 3.3. Let $s_{1}, s_{2}$ be two real numbers. Then, the following three assertions hold.

a) Given $m_{i} \in \mathbb{M}_{s_{i}, 1}(\Xi)(i=1,2)$, we have $m_{1} m_{2} \in \mathbb{M}_{s_{1}+s_{2}, 1}(\Xi)$.

b) Given $\ell_{i} \in \mathbb{M}_{s_{i}, i}(\Xi)(i=1,2)$, we have $\ell_{1} \ell_{2} \in \mathbb{M}_{s_{1}+s_{2}, 2}(\Xi)$.

c) Given $n_{i} \in \mathbb{M}_{s_{i}, 2}(\Xi)(i=1,2)$, we have $n_{1} n_{2} \in \mathbb{M}_{s_{1}+s_{2}, 2}(\Xi)$.

Due to $\partial_{\xi^{\prime}}^{\alpha^{\prime}} \xi_{\ell}=0$ for $\left|\alpha^{\prime}\right| \geq 1$, we have $\left(\xi^{\prime}, \lambda\right) \mapsto \xi_{\ell} \in \mathbb{M}_{1,1}\left(\Sigma_{\vartheta_{0}}\right)$. Similarly, due to $\partial_{\xi^{\prime}}^{\alpha^{\prime}}\left|\xi^{\prime}\right|^{2}=0$ for $\left|\alpha^{\prime}\right| \geq 3$ we obtain for $\zeta:=(1-\beta)\left|\xi^{\prime}\right|^{2}($ see $(3-12))$

$$
\left|\partial_{\xi^{\prime}}^{\alpha^{\prime}} \zeta\right| \leq C\left(|\lambda|^{1 / 2}+\left|\xi^{\prime}\right|\right)^{2-\left|\alpha^{\prime}\right|},
$$

which yields that $\zeta \in \mathbb{M}_{2,1}\left(\Sigma_{\vartheta_{0}}\right)$. Here and in the following, $\vartheta_{0}$ is the number given in (2-6). By (2-7),

$$
c\left(|\lambda|^{1 / 2}+\left|\xi^{\prime}\right|\right) \leq \operatorname{Re} A_{i}\left(\xi^{\prime}, \lambda\right) \leq\left|A_{i}\left(\xi^{\prime}, \lambda\right)\right| \leq C\left(|\lambda|^{1 / 2}+\left|\xi^{\prime}\right|\right)
$$

with some positive constants $c$ and $C$, which furnishes that

$$
A_{i}\left(\xi^{\prime}, \lambda\right)^{s} \in \mathbb{M}_{s, 1}\left(\Sigma_{\vartheta_{0}}\right), \quad\left(A_{i}\left(\xi^{\prime}, \lambda\right)+\left|\xi^{\prime}\right|\right)^{-1} \in \mathbb{M}_{-1,2}\left(\Sigma_{\vartheta_{0}}\right),
$$

where $s$ is any real number. The property of the Lopatinskiu matrix $\Delta$ is given in

Theorem 3.4. There exists a number $\frac{\pi}{2}<\vartheta \leq \pi$ such that the inverse matrix $\Delta\left(\xi^{\prime}, \lambda\right)^{-1}$ exists for any $\lambda \in \Sigma_{\vartheta}$ and $\xi^{\prime} \in \mathbb{R}^{N-1}$. Let

$$
\Delta\left(\xi^{\prime}, \lambda\right)^{-1}=\left(g_{i j}\left(\xi^{\prime}, \lambda\right)\right)_{i, j=1,2,3}
$$

Then,

$$
\lambda g_{i 1} \in \mathbb{M}_{0,1}\left(\Sigma_{\vartheta}\right)(i=1,2,3), \quad \lambda g_{i j} \in \mathbb{M}_{-1,1}\left(\Sigma_{\vartheta}\right)(i=1,2,3, \quad j=2,3) .
$$

Moreover, there exists a positive constant $\sigma_{0}>0$ such that

$$
\begin{aligned}
\lambda g_{i 1} \in \mathbb{M}_{0,2}\left(\Xi_{\vartheta, \sigma_{0}}\right) \quad(i=1,2,3), \quad \sum_{i=1}^{3} g_{i 1} \in \mathbb{M}_{-2,2}\left(\Xi_{\vartheta, \sigma_{0}}\right) \quad(i=1,2,3), \\
\lambda g_{i j} \in \mathbb{M}_{-1,2}\left(\Xi_{\vartheta, \sigma_{0}}\right)(i=1,2,3, \quad j=2,3), \quad \sum_{i=1}^{3} g_{i j} \in \mathbb{M}_{-3,2}\left(\Xi_{\vartheta, \sigma_{0}}\right) \quad(i=1,2,3, j=2,3)
\end{aligned}
$$

with

$$
\Xi_{\vartheta, \sigma_{0}}=\left\{\left(\xi^{\prime}, \lambda\right) \in \mathbb{R}^{N-1} \times\left.\Sigma_{\vartheta}\left|\sigma_{0}\right| \xi^{\prime}|\geq| \lambda\right|^{1 / 2}, \quad \lambda \in \Sigma_{\vartheta}\right\}
$$

The proof of Theorem 3.4 is the highlight of this paper, But, it is postponed to Section 4 and using Theorem 3.4, we are going to investigate the solution operator of the parameter-dependent system (3-6) and (3-2). By (3-9), (3-11) and Theorem 3.4,

$$
\begin{aligned}
& w\left(\xi^{\prime}, x_{N}, \lambda\right)=\sum_{i, j=1}^{3} e^{-A_{i}\left(\xi^{\prime}, \lambda\right) x_{N}} g_{i j}\left(\xi^{\prime}, \lambda\right) \mathscr{F}^{\prime}\left[g_{j}\right]\left(\xi^{\prime}, 0\right) \\
& \tau\left(\xi^{\prime}, x_{N}, \lambda\right)=-\lambda \sum_{i, j=1}^{3} e^{-A_{i}\left(\xi^{\prime}, \lambda\right) x_{N}}\left(\gamma_{i}^{2}+2\right) g_{i j}\left(\xi^{\prime}, \lambda\right) \mathscr{F}^{\prime}\left[g_{j}\right]\left(\xi^{\prime}, 0\right)
\end{aligned}
$$

Let $\psi$ be a $C^{\infty}$ function on $\mathbb{R}$ such that $\psi(t)=1$ for $t<1$ and $\psi(t)=0$ for $t>2$, and set $\varphi_{0}\left(\xi^{\prime}, \lambda\right)=\psi\left(c_{0}\left|\xi^{\prime}\right| /|\lambda|^{1 / 2}\right)$ and $\varphi_{\infty}\left(\xi^{\prime}, \lambda\right)=1-\varphi_{0}\left(\xi^{\prime}, \lambda\right)$. Note that

$$
\varphi_{0}\left(\xi^{\prime}, \lambda\right)=\left\{\begin{array}{ll}
1 & \text { if } c_{0}\left|\xi^{\prime}\right| \leq|\lambda|^{1 / 2}, \\
0 & \text { if } c_{0}\left|\xi^{\prime}\right| \geq 2|\lambda|^{1 / 2},
\end{array} \quad \varphi_{\infty}\left(\xi^{\prime}, \lambda\right)= \begin{cases}0 & \text { if } c_{0}\left|\xi^{\prime}\right| \leq|\lambda|^{1 / 2} \\
1 & \text { if } c_{0}\left|\xi^{\prime}\right| \geq 2|\lambda|^{1 / 2}\end{cases}\right.
$$


In view of Theorem 3.4, we write

$$
\begin{aligned}
& w\left(\xi^{\prime}, x_{N}, \lambda\right)=\sum_{i, j=1}^{3} e^{-A_{i}\left(\xi^{\prime}, \lambda\right) x_{N}} g_{i j}\left(\xi^{\prime}, \lambda\right) \mathscr{F}^{\prime}\left[g_{j}\right]\left(\xi^{\prime}, 0\right), \\
& =\sum_{i, j=1}^{3} e^{-A_{i}\left(\xi^{\prime}, \lambda\right) x_{N}} \varphi_{0}\left(\xi^{\prime}, \lambda\right) g_{i j}\left(\xi^{\prime}, \lambda\right) \mathscr{F}^{\prime}\left[g_{j}\right]\left(\xi^{\prime}, 0\right) \\
& +\sum_{i, j=1}^{3} e^{-A_{i}\left(\xi^{\prime}, \lambda\right) x_{N}} \varphi_{\infty}\left(\xi^{\prime}, \lambda\right) g_{i j}\left(\xi^{\prime}, \lambda\right) \mathscr{F}^{\prime}\left[g_{j}\right]\left(\xi^{\prime}, 0\right) \\
& =\sum_{i, j=1}^{3} e^{-A_{i}\left(\xi^{\prime}, \lambda\right) x_{N}} \varphi_{0}\left(\xi^{\prime}, \lambda\right) g_{i j}\left(\xi^{\prime}, \lambda\right) \mathscr{F}^{\prime}\left[g_{j}\right]\left(\xi^{\prime}, 0\right) \\
& +\sum_{i, j=1}^{3}\left(e^{-A_{i}\left(\xi^{\prime}, \lambda\right) x_{N}}-e^{-\left|\xi^{\prime}\right| x_{N}}\right) \varphi_{\infty}\left(\xi^{\prime}, \lambda\right) g_{i j}\left(\xi^{\prime}, \lambda\right) \mathscr{F}^{\prime}\left[g_{j}\right]\left(\xi^{\prime}, 0\right) \\
& +\sum_{j=1}^{3} e^{-\left|\xi^{\prime}\right|\left(\xi^{\prime}, \lambda\right) x_{N}}\left\{\varphi_{\infty}\left(\xi^{\prime}, \lambda\right) \sum_{i=1}^{3} g_{i j}\left(\xi^{\prime}, \lambda\right)\right\} \mathscr{F}^{\prime}\left[g_{j}\right]\left(\xi^{\prime}, 0\right) .
\end{aligned}
$$

In what follows, let $A=\left|\xi^{\prime}\right|$ for short, and let

$$
\mathcal{M}_{k}=\mathcal{M}_{k}\left(\xi^{\prime}, x_{N}, \lambda\right):=\frac{e^{-A_{k}\left(\xi^{\prime}, \lambda\right) x_{N}}-e^{-A x_{N}}}{A_{k}\left(\xi^{\prime}, \lambda\right)-A} \quad(k=1,2,3) .
$$

We have the identities

$$
\begin{aligned}
& \partial_{N} \mathcal{M}_{k}\left(\xi^{\prime}, x_{N}, \lambda\right)=-e^{-A_{k}\left(\xi^{\prime}, \lambda\right) x_{N}}-A \mathcal{M}_{k}\left(\xi^{\prime}, x_{N}, \lambda\right) \\
& \partial_{N}^{2} \mathcal{M}_{k}\left(\xi^{\prime}, x_{N}, \lambda\right)=\left(A_{k}\left(\xi^{\prime}, \lambda\right)+A\right) e^{-A_{k}\left(\xi^{\prime}, \lambda\right) x_{N}}+A^{2} \mathcal{M}_{k}\left(\xi^{\prime}, x_{N}, \lambda\right) .
\end{aligned}
$$

Let $\mathscr{F}^{\prime-1}[\varphi]$ denote the inverse partial Fourier transform defined by

$$
\mathscr{F}^{\prime-1}[\varphi]\left(x^{\prime}, x_{N}\right):=\frac{1}{(2 \pi)^{n-1}} \int_{\mathbb{R}^{n-1}} e^{i x^{\prime} \cdot \xi^{\prime}} \varphi\left(\xi^{\prime}, x_{N}\right) d \xi^{\prime} .
$$

Let

$$
u(x, \lambda)=\mathscr{F}^{\prime-1}\left[w\left(\xi^{\prime}, x_{N}, \lambda\right)\right], \quad \theta(x, \lambda)=\mathscr{F}^{\prime-1}\left[\tau\left(\xi^{\prime}, x_{N}, \lambda\right)\right]
$$

First, we treat $u(x, \lambda)$. Using the Volevich trick and (3-23) and applying the inverse partial Fourier transform, we rewrite $u(x, \lambda)=\sum_{k=1}^{6} T_{j}(\lambda) g, g=\left(g_{1}, g_{2}, g_{3}\right)^{\top}$, with

$$
\begin{aligned}
& T_{1}(\lambda) g(x)=-\int_{0}^{\infty} \mathscr{F}^{\prime-1}\left[\sum_{i, j=1}^{3} g_{i j}\left(\xi^{\prime}, \lambda\right) \varphi_{0}\left(\xi^{\prime}, \lambda\right) e^{\left.-A_{i}\left(\xi^{\prime}, \lambda\right)\left(x_{N}+y_{N}\right) \mathscr{F}^{\prime}\left[\partial_{N} g_{j}\right]\left(\xi^{\prime}, y_{N}\right)\right]\left(x^{\prime}\right) d y_{N}}\right. \\
& T_{2}(\lambda) g(x)=\int_{0}^{\infty} \mathscr{F}^{\prime-1}\left[\sum_{i, j=1}^{3} g_{i j}\left(\xi^{\prime}, \lambda\right) \varphi_{0}\left(\xi^{\prime}, \lambda\right) e^{-A_{i}\left(\xi^{\prime}, \lambda\right)\left(x_{N}+y_{N}\right)} A_{i}\left(\xi^{\prime}, \lambda\right) \mathscr{F}^{\prime}\left[g_{j}\right]\left(\xi^{\prime}, y_{N}\right)\right]\left(x^{\prime}\right) d y_{N}, \\
& T_{3}(\lambda) g(x)=-\int_{0}^{\infty} \mathscr{F}^{\prime-1}\left[\sum_{i, j=1}^{3} g_{i j}\left(\xi^{\prime}, \lambda\right) \varphi_{\infty}\left(\xi^{\prime}, \lambda\right)\left(e^{-A_{i}\left(\xi^{\prime}, \lambda\right)\left(x_{N}+y_{N}\right)}-e^{-A\left(x_{N}+y_{N}\right)}\right)\right. \\
& T_{4}(\lambda) g(x)=\int_{0}^{\infty} \mathscr{F}^{\prime-1}\left[\sum _ { i , j = 1 } ^ { 3 } g _ { i j } ( \xi ^ { \prime } , \lambda ) \varphi _ { \infty } ( \xi ^ { \prime } , \lambda ) \left\{\left(\partial_{i}\left(\xi^{\prime}, \lambda\right)-A\right) e^{-A_{i}\left(\xi^{\prime}, \lambda\right)\left(x_{N}+y_{N}\right)},\right.\right.
\end{aligned}
$$




$$
\begin{array}{r}
\left.\left.+A\left(e^{-A_{i}\left(\xi^{\prime}, \lambda\right)\left(x_{N}+y_{N}\right)}-e^{-A\left(x_{N}+y_{N}\right)}\right)\right\} \mathscr{F}^{\prime}\left[g_{j}\right]\left(\xi^{\prime}, y_{N}\right)\right]\left(x^{\prime}\right) d y_{N} \\
T_{5}(\lambda) g(x)=-\int_{0}^{\infty} \mathscr{F}^{\prime-1}\left[\sum_{j=1}^{3}\left(\sum_{i=1}^{3} g_{i j}\left(\xi^{\prime}, \lambda\right)\right) \varphi_{\infty}\left(\xi^{\prime}, \lambda\right) e^{\left.-A\left(x_{N}+y_{N}\right) \mathscr{F}^{\prime}\left[\partial_{N} g_{j}\right]\left(\xi^{\prime}, y_{N}\right)\right]\left(x^{\prime}\right) d y_{N},}\right. \\
T_{6}(\lambda) g(x)=\int_{0}^{\infty} \mathscr{F}^{\prime-1}\left[\sum_{j=1}^{3}\left(\sum_{i=1}^{3} g_{i j}\left(\xi^{\prime}, \lambda\right)\right) \varphi_{\infty}\left(\xi^{\prime}, \lambda\right) A e^{-A\left(x_{N}+y_{N}\right)} \mathscr{F}^{\prime}\left[g_{j}\right]\left(\xi^{\prime}, y_{N}\right)\right]\left(x^{\prime}\right) d y_{N} .
\end{array}
$$

To construct $\mathcal{R}$-bounded solution operators associated with problem (3-1) and (3-2), we use the following lemma due to Shibata and Shimizu [30, Lemma 5.6].

Lemma 3.5. Let $\vartheta>\pi / 2$ be the same number as in Theorem 3.4 and let $A_{k}\left(\xi^{\prime}, \lambda\right)$ and $\mathcal{M}_{k}\left(\xi^{\prime}, x_{N}, \lambda\right)$ $(k=1,2,3)$ be functions given in (3-10) and (3-22), respectively. Given $\ell_{0}\left(\xi,{ }^{\prime}, \lambda\right) \in \mathbb{M}_{-2,1}\left(\Sigma_{\vartheta}\right)$ and $\ell_{1}\left(\xi^{\prime}, \lambda\right) \in \mathbb{M}_{-2,2}\left(\Sigma_{\vartheta}\right)$, we define the operators $L_{j}(\lambda)(j=1,2,3,4)$ by

$$
\begin{aligned}
& {\left[L_{1}(\lambda) h\right](x)=\int_{0}^{\infty} \mathscr{F}^{-1}\left[\ell_{0}\left(\xi^{\prime}, \lambda\right) \lambda^{1 / 2} e^{-A_{k}\left(\xi^{\prime}, \lambda\right)\left(x_{N}+y_{N}\right) \mathscr{F}^{\prime}}[h]\left(\xi^{\prime}, y_{N}\right)\right]\left(x^{\prime}\right) d y_{N},} \\
& {\left[L_{2}(\lambda) h\right](x)=\int_{0}^{\infty} \mathscr{F}^{\prime-1}\left[\ell_{1}(\xi, \lambda) A e^{\left.-A_{k}\left(\xi^{\prime}, \lambda\right)\left(x_{N}+y_{N}\right) \mathscr{F}^{\prime}[h]\left(\xi^{\prime}, y_{N}\right)\right]\left(x^{\prime}\right) d y_{N},}\right.} \\
& {\left[L_{3}(\lambda) h\right](x)=\int_{0}^{\infty} \mathscr{F}^{\prime-1}\left[\ell_{1}\left(\xi^{\prime}, \lambda\right) A e^{\left.-A\left(x_{N}+y_{N}\right) \mathscr{F}^{\prime}[h]\left(\xi^{\prime}, y_{N}\right)\right]\left(x^{\prime}\right) d y_{N},}\right.} \\
& {\left[L_{4}(\lambda) h\right](x)=\int_{0}^{\infty} \mathscr{F}^{\prime-1}\left[\ell_{1}\left(\xi^{\prime}, \lambda\right) A^{2} \mathcal{M}_{k}\left(\xi^{\prime}, x_{N}+y_{N}, \lambda\right) \mathscr{F}^{\prime}[h]\left(\xi^{\prime}, y_{N}\right)\right]\left(x^{\prime}\right) d y_{N} .}
\end{aligned}
$$

Then,

$$
\mathcal{R}_{\mathcal{L}\left(L_{q}\left(\mathbb{R}_{+}^{N}\right)\right)}\left(\left\{\left(\tau \partial_{\tau}\right)^{s}\left(\lambda^{j / 2} \partial_{x}^{\alpha} L_{i}(\lambda)\right) \mid \lambda \in \Sigma_{\vartheta}\right\}\right) \leq C_{N, q} \quad(s=0,1, \quad j+|\alpha|=2, \quad i=1,2,3,4) .
$$

From Lemma 3.5 and Lemma 3.3 the following lemma follows immediately.

Lemma 3.6. Let $\vartheta>\pi / 2$ be the same number as in Theorem 3.4 and let $A_{k}\left(\xi^{\prime}, \lambda\right)$ and $M_{k}\left(\xi^{\prime}, x_{N}, \lambda\right)$ $(k=1,2,3)$ be functions given in (3-10) and (3-22), respectively. Given $m_{0}\left(\xi^{\prime}, \lambda\right) \in \mathbb{M}_{-4,1}\left(\Sigma_{\vartheta}\right)$ and $m_{1}\left(\xi^{\prime}, \lambda\right) \in \mathbb{M}_{-4,2}\left(\Sigma_{\vartheta}\right)$, we define the operators $M_{j}(\lambda)(j=1,2,3,4)$ by

$$
\begin{aligned}
& {\left[M_{1}(\lambda) h\right](x)=\int_{0}^{\infty} \mathscr{F}^{\prime-1}\left[m_{0}\left(\xi^{\prime}, \lambda\right) \lambda^{1 / 2} e^{\left.-A_{k}\left(\xi^{\prime}, \lambda\right)\left(x_{N}+y_{N}\right) \mathscr{F}^{\prime}[h]\left(\xi^{\prime}, y_{N}\right)\right]\left(x^{\prime}\right) d y_{N},}\right.} \\
& {\left[M_{2}(\lambda) h\right](x)=\int_{0}^{\infty} \mathscr{F}^{\prime-1}\left[m_{1}\left(\xi^{\prime}, \lambda\right) A e^{\left.-A_{k}\left(\xi^{\prime}, \lambda\right)\left(x_{N}+y_{N}\right) \mathscr{F}^{\prime}[h]\left(\xi^{\prime}, y_{N}\right)\right]\left(x^{\prime}\right) d y_{N},}\right.} \\
& {\left[M_{3}(\lambda) h\right](x)=\int_{0}^{\infty} \mathscr{F}^{\prime-1}\left[m_{1}\left(\xi^{\prime}, \lambda\right) A e^{\left.-A\left(x_{N}+y_{N}\right) \mathscr{F}^{\prime}[h]\left(\xi^{\prime}, y_{N}\right)\right]\left(x^{\prime}\right) d y_{N},}\right.} \\
& {\left[M_{4}(\lambda) h\right](x)=\int_{0}^{\infty} \mathscr{F}^{\prime-1}\left[m_{1}\left(\xi^{\prime}, \lambda\right) A^{2} \mathcal{M}_{k}\left(\xi^{\prime}, x_{N}+y_{N}, \lambda\right) \mathscr{F}^{\prime}[h]\left(\xi^{\prime}, y_{N}\right)\right]\left(x^{\prime}\right) d y_{N} .}
\end{aligned}
$$

Then,

$$
\mathcal{R}_{\mathcal{L}\left(L_{q}\left(\mathbb{R}_{+}^{N}\right)\right)}\left(\left\{\left(\tau \partial_{\tau}\right)^{s}\left(\lambda^{j / 2} \partial_{x}^{\alpha} M_{i}(\lambda)\right) \mid \lambda \in \Sigma_{\vartheta}\right\}\right) \leq C_{N, q} \quad(s=0,1, \quad j+|\alpha|=4, \quad i=1,2,3,4) .
$$

Proof. Since $\lambda m_{0}\left(\xi^{\prime}, \lambda\right) \in \mathbb{M}_{-2,2}\left(\Sigma_{\vartheta}\right)$, it follows from Lemma 3.5 that (3-24) holds for $i=1$ and $j+|\alpha|=4$ with $j=2,3,4$. Let $a, b \in\{1, \ldots, N-1\}$. We have

$$
\begin{aligned}
& \partial_{a} \partial_{b}\left[M_{1}(\lambda) h\right](x)=-\int_{0}^{\infty} \mathscr{F}^{\prime-1}\left[\xi_{a} \xi_{b} m_{0}\left(\lambda, \xi^{\prime}\right) \lambda^{1 / 2} e^{-A_{i}\left(\xi^{\prime}, \lambda\right)\left(x_{N}+y_{N}\right)} \mathscr{F}^{\prime}[h]\left(\xi^{\prime}, y_{N}\right)\right]\left(x^{\prime}\right) d y_{N}, \\
& \partial_{a} \partial_{N}\left[M_{1}(\lambda) h\right](x)=-i \int_{0}^{\infty} \mathscr{F}^{\prime-1}\left[\xi_{a} A_{i}\left(\xi^{\prime}, \lambda\right) m_{0}\left(\lambda, \xi^{\prime}\right) \lambda^{1 / 2} e^{\left.-A_{i}\left(\xi^{\prime}, \lambda\right)\left(x_{N}+y_{N}\right) \mathscr{F}^{\prime}[h]\left(\xi^{\prime}, y_{N}\right)\right]\left(x^{\prime}\right) d y_{N},}\right.
\end{aligned}
$$




$$
\partial_{N}^{2}\left[M_{1}(\lambda) h\right](x)=\int_{0}^{\infty} \mathscr{F}^{\prime-1}\left[A_{i}\left(\xi^{\prime}, \lambda\right)^{2} m_{0}\left(\lambda, \xi^{\prime}\right) \lambda^{1 / 2} e^{-A_{i}\left(\xi^{\prime}, \lambda\right)\left(x_{N}+y_{N}\right)} \mathscr{F}^{\prime}[h]\left(\xi^{\prime}, y_{N}\right)\right]\left(x^{\prime}\right) d y_{N} .
$$

Since $\xi_{a}$ and $A_{k}\left(\xi^{\prime}, \lambda\right) \in \mathbb{M}_{1,1}\left(\Sigma_{\vartheta}\right)$, it follows from Lemma 3.3 that $\xi_{a} \xi_{b} m_{0}\left(\xi^{\prime}, \lambda\right), \xi_{a} A_{k}\left(\xi^{\prime}, \lambda\right) m_{0}\left(\xi^{\prime}, \lambda\right)$, and $A_{k}\left(\xi^{\prime}, \lambda\right)^{2} m_{0}\left(\xi^{\prime}, \lambda\right)$ belong to $\mathbb{M}_{-2,1}\left(\Sigma_{\vartheta}\right)$, so that it follows from Lemma 3.5 that (3-24) holds for $i=1$ and $j+|\alpha|=4$ with $|\alpha|=2,3$, 4 . Since $\lambda m_{1}\left(\xi^{\prime}, \lambda\right) \in \mathbb{M}_{-2,2}\left(\Sigma_{\vartheta}\right)$, it follows from Lemma 3.5 that (3-24) holds for $i=2,3,4$ and $j+|\alpha|=4$ with $j=2,3,4$. By Lemma 3.3, $\xi_{a} \xi_{b} m_{1}\left(\xi^{\prime}, \lambda\right), \xi_{a} A_{k}\left(\xi^{\prime}, \lambda\right) m_{1}\left(\xi^{\prime}, \lambda\right)$, $A_{k}\left(\xi^{\prime}, \lambda\right)^{2} m_{1}\left(\xi^{\prime}, \lambda\right), \xi_{a} A m_{1}\left(\xi^{\prime}, \lambda\right)$, and $A^{2} m_{1}\left(\xi^{\prime}, \lambda\right)$ belong to $\mathbb{M}_{-2,2}\left(\Sigma_{\vartheta}\right)$. It follows from Lemma 3.5 that (3-24) holds for $i=2,3$ and $j+|\alpha|=4$ with $|\alpha|=2,3,4$. Since $\xi_{a} \xi_{b} m_{1}\left(\xi^{\prime}, \lambda\right), \xi_{a} A m_{1}\left(\xi^{\prime}, \lambda\right)$, $\left(A_{k}\left(\xi^{\prime}, \lambda\right)+A\right) A m_{1}\left(\xi^{\prime}, \lambda\right)$, and $A^{2} m_{1}\left(\xi^{\prime}, \lambda\right)$ belong to $\mathbb{M}_{-2,2}\left(\Sigma_{\vartheta}\right)$, it follows from Lemma 3.5 that (3-24) holds for $i=4$ and $j+|\alpha|=4$ with $|\alpha|=2,3$, , which completes the proof of Lemma 3.6.

To define the solution operators, we change the formulas $T_{j}(\lambda)$. First of all, we rewrite $T_{1}(\lambda) g$ as follows:

$$
\begin{aligned}
T_{1}(\lambda) g(x)=-\int_{0}^{\infty} \mathscr{F}^{\prime-1}\left[\sum _ { k = 1 } ^ { 3 } \left(\lambda^{-1} g_{k 1}\left(\xi^{\prime}, \lambda\right) \varphi_{0}\left(\xi^{\prime}, \lambda\right) \mathscr{F}^{\prime}\left[\lambda^{1 / 2} \partial_{N} g_{1}\right]\left(\xi^{\prime}, y_{N}\right)\right.\right. \\
\left.\left.+\sum_{j=2}^{3} \lambda^{-1 / 2} g_{k j}\left(\xi^{\prime}, \lambda\right) \varphi_{0}\left(\xi^{\prime}, \lambda\right) \mathscr{F}^{\prime}\left[\partial_{N} g_{j}\right]\left(\xi^{\prime}, y_{N}\right)\right) \lambda^{1 / 2} e^{-A_{k}\left(\xi^{\prime}, \lambda\right)\left(x_{N}+y_{N}\right)}\right]\left(x^{\prime}\right) d y_{N} .
\end{aligned}
$$

Recall that $G^{\prime}=\left(g_{1}^{\prime}, g_{2}^{\prime}, g_{3}^{\prime}\right)$ and $g_{1}^{\prime \prime}$ correspond to $\lambda^{1 / 2} G=\lambda^{1 / 2}\left(g_{1}, g_{2}, g_{3}\right)$ and $\lambda g_{1}$, respectively. Let

$$
\mathcal{X}_{q}^{\prime}(D)=\left\{\left(G, G^{\prime}, g_{1}^{\prime \prime}\right) \mid G \in H_{q}^{2}(D) \times H_{q}^{1}(D)^{2}, G^{\prime} \in H_{q}^{1}(D) \times L_{q}(D)^{2}, g_{1}^{\prime \prime} \in L_{q}(D)\right\},
$$

and let

$$
\begin{aligned}
\mathcal{U}_{1}(\lambda)\left(G, G^{\prime}, g_{1}^{\prime \prime}\right)(x)= & -\int_{0}^{\infty} \mathscr{F}^{\prime-1}\left[\sum _ { k = 1 } ^ { 3 } \left(\lambda^{-1} g_{k 1}\left(\xi^{\prime}, \lambda\right) \varphi_{0}\left(\xi^{\prime}, \lambda\right) \mathscr{F}^{\prime}\left[\partial_{N} g_{1}^{\prime}\right]\left(\xi^{\prime}, y_{N}\right)\right.\right. \\
& \left.\left.+\sum_{j=2}^{3} \lambda^{-1 / 2} g_{k j}\left(\xi^{\prime}, \lambda\right) \varphi_{0}\left(\xi^{\prime}, \lambda\right) \mathscr{F}^{\prime}\left[\partial_{N} g_{j}\right]\left(\xi^{\prime}, y_{N}\right)\right) \lambda^{1 / 2} e^{-A_{k}\left(\xi^{\prime}, \lambda\right)\left(x_{N}+y_{N}\right)}\right]\left(x^{\prime}\right) d y_{N} .
\end{aligned}
$$

Obviously, $T_{1}(\lambda) g=\mathcal{U}_{1}(\lambda)\left(g, \lambda^{1 / 2} g, \lambda g_{1}\right)$. Moreover, it follows from Theorem 3.4 and (3-21) that $\lambda^{-1} g_{k 1}\left(\xi^{\prime}, \lambda\right) \varphi_{0}\left(\xi^{\prime}, \lambda\right)$ and $\lambda^{-1 / 2} g_{k j}\left(\xi^{\prime}, \lambda\right) \varphi_{0}\left(\xi^{\prime}, \lambda\right)(j=2,3)$ belong to $\mathbb{M}_{-4,1}\left(\Sigma_{\vartheta}\right)$, which, combined with Lemma 3.6, furnishes that

$$
\mathcal{R}_{\mathcal{L}\left(\mathcal{X}_{q}^{\prime}\left(\mathbb{R}_{+}^{N}\right), L_{q}\left(\mathbb{R}_{+}^{N}\right)\right)}\left(\left\{\left(\tau \partial_{\tau}\right)^{s}\left(\lambda^{j / 2} \partial_{x}^{\alpha} \mathcal{U}_{1}(\lambda)\right) \mid \lambda \in \Sigma_{\vartheta}\right\}\right) \leq C_{N, q} \quad(s=0,1)
$$

for any $j \in \mathbb{N}_{0}$ and $\alpha \in \mathbb{N}_{0}^{N}$ with $j+|\alpha|=4$.

Next, analogously to $T_{1}(\lambda) g$, we rewrite $T_{2}(\lambda) g$ as follows:

$$
\begin{aligned}
T_{2}(\lambda) g(x)= & \int_{0}^{\infty} \mathscr{F}^{\prime-1}\left[\sum _ { k = 1 } ^ { 3 } \left(g_{k 1}\left(\xi^{\prime}, \lambda\right) \varphi_{0}\left(\xi^{\prime}, \lambda\right) A_{k}\left(\xi^{\prime}, \lambda\right) \lambda^{-3 / 2} \mathscr{F}^{\prime}\left[\lambda g_{1}\right]\left(\xi^{\prime}, y_{N}\right)\right.\right. \\
& \left.\left.+\sum_{j=2}^{3} g_{k j}\left(\xi^{\prime}, \lambda\right) \varphi_{0}\left(\xi^{\prime}, \lambda\right) A_{k}\left(\xi^{\prime}, \lambda\right) \lambda^{-1} \mathscr{F}^{\prime}\left[\lambda^{1 / 2} g_{j}\right]\left(\xi^{\prime}, y_{N}\right)\right) \lambda^{1 / 2} e^{-A_{k}\left(\xi^{\prime}, \lambda\right)\left(x_{N}+y_{N}\right)}\right]\left(x^{\prime}\right) d y_{N} .
\end{aligned}
$$

Let

$$
\begin{aligned}
\mathcal{U}_{2}(\lambda)\left(G, G^{\prime}, g_{1}^{\prime \prime}\right)(x) & =\int_{0}^{\infty} \mathscr{F}^{\prime-1}\left[\sum _ { k = 1 } ^ { 3 } \left(g_{k 1}\left(\xi^{\prime}, \lambda\right) \varphi_{0}\left(\xi^{\prime}, \lambda\right) A_{k}\left(\xi^{\prime}, \lambda\right) \lambda^{-3 / 2} \mathscr{F}^{\prime}\left[g_{1}^{\prime \prime}\right]\left(\xi^{\prime}, y_{N}\right)\right.\right. \\
+ & \left.\left.\sum_{j=2}^{3} g_{k j}\left(\xi^{\prime}, \lambda\right) \varphi_{0}\left(\xi^{\prime}, \lambda\right) A_{k}\left(\xi^{\prime}, \lambda\right) \lambda^{-1} \mathscr{F}^{\prime}\left[g_{j}^{\prime}\right]\left(\xi^{\prime}, y_{N}\right)\right) \lambda^{1 / 2} e^{-A_{k}\left(\xi^{\prime}, \lambda\right)\left(x_{N}+y_{N}\right)}\right]\left(x^{\prime}\right) d y_{N} .
\end{aligned}
$$


Obviously, $T_{2}(\lambda) g=\mathcal{U}_{2}(\lambda)\left(g, \lambda^{1 / 2} g, \lambda g_{1}\right)$. Moreover, it follows from Theorem 3.4 and $(3-21)$ that $g_{k 1}\left(\xi^{\prime}, \lambda\right) \varphi_{0}\left(\xi^{\prime}, \lambda\right) A_{k}\left(\xi^{\prime}, \lambda\right) \lambda^{-3 / 2}$ and $g_{k j}\left(\xi^{\prime}, \lambda\right) \varphi_{0}\left(\xi^{\prime}, \lambda\right) A_{k}\left(\xi^{\prime}, \lambda\right) \lambda^{-1}(j=2,3)$ belong to $\mathbb{M}_{-4,1}\left(\Sigma_{\vartheta}\right)$, which, combined with Lemma 3.6, furnishes that

$$
\mathcal{R}_{\mathcal{L}\left(\mathcal{X}_{q}^{\prime}\left(\mathbb{R}_{+}^{N}\right), L_{q}\left(\mathbb{R}_{+}^{N}\right)\right)}\left(\left\{\left(\tau \partial_{\tau}\right)^{s}\left(\lambda^{j / 2} \partial_{x}^{\alpha} \mathcal{U}_{2}(\lambda)\right) \mid \lambda \in \Sigma_{\vartheta}\right\}\right) \leq C_{N, q} \quad(s=0,1)
$$

for any $j \in \mathbb{N}_{0}$ and $\alpha \in \mathbb{N}_{0}^{N}$ with $j+|\alpha|=4$.

Next, we consider $T_{3}(\lambda) g$. Using the identities

$$
\lambda=\gamma_{k}\left(A_{k}\left(\xi^{\prime}, \lambda\right)+A\right)\left(A_{k}\left(\xi^{\prime}, \lambda\right)-A\right), \quad 1=-\sum_{\ell=1}^{N-1}\left(i \xi_{\ell}\right)\left(i \xi_{\ell}\right) A^{-2}
$$

we rewrite $T_{3}(\lambda) g$ as follows:

$$
\begin{aligned}
& T_{3}(\lambda) g(x)=\gamma_{k}^{-1} \int_{0}^{\infty} \mathscr{F}^{\prime-1}\left[\sum _ { k = 1 } ^ { 3 } \left(\sum_{\ell=1}^{N-1}\left[\lambda g_{k 1}\left(\xi^{\prime}, \lambda\right) \varphi_{\infty}\left(\xi^{\prime}, \lambda\right)\left(A_{k}\left(\xi^{\prime}, \lambda\right)+A\right)^{-1}\left(i \xi_{\ell} A^{-4}\right) \mathscr{F}^{\prime}\left[\partial_{\ell} \partial_{N} g_{1}\left(\xi^{\prime}, y_{N}\right)\right]\right]\right.\right. \\
& \left.\left.\quad-\sum_{j=2}^{3} \lambda g_{i j}\left(\xi^{\prime}, \lambda\right) \varphi_{\infty}\left(\xi^{\prime}, \lambda\right)\left(A_{k}\left(\xi^{\prime}, \lambda\right)+A\right)^{-1} A^{-2} \mathscr{F}^{\prime}\left[\partial_{N} g_{j}\right]\left(\xi^{\prime}, y_{N}\right)\right) A^{2} \mathcal{M}_{k}\left(\xi^{\prime}, x_{N}+y_{N}, \lambda\right)\right]\left(x^{\prime}\right) d y_{N} .
\end{aligned}
$$

Let, $\mathcal{U}_{3}(\lambda)\left(G, G^{\prime}, g_{1}^{\prime \prime}\right)=T_{3}(\lambda) g$. Since the multipliers symbols:

$$
\lambda g_{k 1}\left(\xi^{\prime}, \lambda\right) \varphi_{\infty}\left(\xi^{\prime}, \lambda\right)\left(A_{k}\left(\xi^{\prime}, \lambda\right)+A\right)^{-1}\left(i \xi_{\ell} A^{-4}\right), \lambda g_{i j}\left(\xi^{\prime}, \lambda\right) \varphi_{\infty}\left(\xi^{\prime}, \lambda\right)\left(A_{k}\left(\xi^{\prime}, \lambda\right)+A\right)^{-1} A^{-2}
$$

$(j=2,3)$ belong to $\mathbb{M}_{-4,2}\left(\Sigma_{\vartheta}\right)$ as follows from Theorem 3.4 and (3-21), by Lemma 3.6 we have

$$
\mathcal{R}_{\mathcal{L}\left(\mathcal{X}_{q}^{\prime}\left(\mathbb{R}_{+}^{N}\right), L_{q}\left(\mathbb{R}_{+}^{N}\right)\right)}\left(\left\{\left(\tau \partial_{\tau}\right)^{s}\left(\lambda^{j / 2} \partial_{x}^{\alpha} \mathcal{U}_{3}(\lambda)\right) \mid \lambda \in \Sigma_{\vartheta}\right\}\right) \leq C_{N, q} \quad(s=0,1)
$$

for any $j \in \mathbb{N}_{0}$ and $\alpha \in \mathbb{N}_{0}^{N}$ with $j+|\alpha|=4$.

Using (3-27), we also have

$$
\begin{aligned}
T_{4}(\lambda) g(x)=- & \int_{0}^{\infty} \mathscr{F}^{\prime-1}\left[\sum _ { k = 1 } ^ { 3 } \gamma _ { k } ^ { - 1 } \left\{\left(\lambda g_{k 1}\left(\xi^{\prime}, \lambda\right) \varphi_{\infty}\left(\xi^{\prime}, \lambda\right)\left(A_{k}\left(\xi^{\prime}, \lambda\right)+A\right)^{-1} A^{-3} \mathscr{F}^{\prime}\left[\Delta g_{1}\right]\left(\xi^{\prime}, y_{N}\right)\right.\right.\right. \\
+ & \left.\sum_{j=2}^{3} \sum_{\ell=1}^{N-1} \lambda g_{k j}\left(\xi^{\prime}, \lambda\right) \varphi_{\infty}\left(\xi^{\prime}, \lambda\right)\left(A_{k}\left(\xi^{\prime}, \lambda\right)+A\right)^{-1} A^{-3}\left(i \xi_{\ell}\right) \mathscr{F}^{\prime}\left[\partial_{\ell} g_{j}\right]\left(\xi^{\prime}, y_{N}\right)\right) \\
& \left.\left.\left(A e^{-A_{k}\left(\xi^{\prime}, \lambda\right)\left(x_{N}+y_{N}\right)}+A^{2} \mathcal{M}_{k}\left(\xi^{\prime}, x_{N}+y_{N}, \lambda\right)\right)\right\}\right]\left(x^{\prime}\right) d y_{N} .
\end{aligned}
$$

Let $\mathcal{U}_{4}(\lambda)\left(G, G^{\prime}, g_{1}^{\prime \prime}\right)=T_{4}(\lambda) g$. Since the multipliers

$$
\lambda g_{k 1}\left(\xi^{\prime}, \lambda\right) \varphi_{\infty}\left(\xi^{\prime}, \lambda\right)\left(A_{k}\left(\xi^{\prime}, \lambda\right)+A\right)^{-1} A^{-3}, \lambda g_{k j}\left(\xi^{\prime}, \lambda\right) \varphi_{\infty}\left(\xi^{\prime}, \lambda\right)\left(A_{k}\left(\xi^{\prime}, \lambda\right)+A\right)^{-1} A^{-3}\left(i \xi_{\ell}\right)
$$

$(j=2,3)$ belong to $\mathbb{M}_{-4,2}\left(\Sigma_{\vartheta}\right)$ as follows from Theorem 3.4 and (3-21), by Lemma 3.6 we have

$$
\mathcal{R}_{\mathcal{L}\left(\mathcal{X}_{q}^{\prime}\left(\mathbb{R}_{+}^{N}\right), L_{q}\left(\mathbb{R}_{+}^{N}\right)\right)}\left(\left\{\left(\tau \partial_{\tau}\right)^{s}\left(\lambda^{j / 2} \partial_{x}^{\alpha} \mathcal{U}_{4}(\lambda)\right) \mid \lambda \in \Sigma_{\vartheta}\right\}\right) \leq C_{N, q} \quad(s=0,1)
$$

for any $j \in \mathbb{N}_{0}$ and $\alpha \in \mathbb{N}_{0}^{N}$ with $j+|\alpha|=4$.

Using the second indentity in (3-27), we rewrite $T_{5}(\lambda) g$ as follows:

$$
T_{5}(\lambda) g(x)=\int_{0}^{\infty} \mathscr{F}^{\prime-1}\left[\left\{\left(\sum_{k=1}^{3} g_{k 1}\left(\xi^{\prime}, \lambda\right)\right) \varphi_{\infty}\left(\xi^{\prime}, \lambda\right) A^{-3} \sum_{\ell=1}^{N-1} i \xi_{\ell} \mathscr{F}^{\prime}\left[\partial_{\ell} \partial_{N} g_{1}\right]\left(\xi^{\prime}, y_{N}\right)\right.\right.
$$




$$
\left.\left.+\sum_{j=2}^{3}\left(\sum_{k=1}^{3} g_{k j}\left(\xi^{\prime}, \lambda\right)\right) \varphi_{\infty}\left(\xi^{\prime}, \lambda\right) A^{-1} \mathscr{F}^{\prime}\left[\partial_{N} g_{j}\right]\left(\xi^{\prime}, y_{N}\right)\right\} A e^{-A\left(x_{N}+y_{N}\right)}\right]\left(x^{\prime}\right) d y_{N} .
$$

Let $\mathcal{U}_{5}(\lambda)\left(G, G^{\prime}, g_{1}^{\prime \prime}\right)=T_{5}(\lambda) g$. Since the multipliers:

$$
\left(\sum_{k=1}^{3} g_{k 1}\left(\xi^{\prime}, \lambda\right)\right) \varphi_{\infty}\left(\xi^{\prime}, \lambda\right) A^{-3}\left(i \xi_{\ell}\right), \quad\left(\sum_{k=1}^{3} g_{k j}\left(\xi^{\prime}, \lambda\right)\right) \varphi_{\infty}\left(\xi^{\prime}, \lambda\right) A^{-1}
$$

$(j=2,3, \quad \ell=1, \ldots, N-1)$ belong to $\mathbb{M}_{-4,2}\left(\Sigma_{\vartheta}\right)$ as follows from Theorem 3.4 and (3-21), by Lemma 3.6 we have

$$
\mathcal{R}_{\mathcal{L}\left(\mathcal{X}_{q}^{\prime}\left(\mathbb{R}_{+}^{N}\right), L_{q}\left(\mathbb{R}_{+}^{N}\right)\right)}\left(\left\{\left(\tau \partial_{\tau}\right)^{s}\left(\lambda^{j / 2} \partial_{x}^{\alpha} \mathcal{U}_{5}(\lambda)\right) \mid \lambda \in \Sigma_{\vartheta}\right\}\right) \leq C_{N, q} \quad(s=0,1)
$$

for any $j \in \mathbb{N}_{0}$ and $\alpha \in \mathbb{N}_{0}^{N}$ with $j+|\alpha|=4$.

Finally, using the second identity in (3-27), we rewrite $T_{6}(\lambda) g$ as follows:

$$
\begin{aligned}
T_{6}(\lambda) g(x)=- & \int_{0}^{\infty} \mathscr{F}^{\prime-1}\left[\left\{\left(\sum_{k=1}^{3} g_{k 1}\left(\xi^{\prime}, \lambda\right)\right) \varphi_{\infty}\left(\xi^{\prime}, \lambda\right) A^{-2} \mathscr{F}^{\prime}\left[\Delta g_{1}\right]\left(\xi^{\prime}, y_{N}\right)\right.\right. \\
& \left.\left.+\sum_{j=2}^{3} \sum_{\ell=1}^{N-1}\left(\sum_{k=1}^{3} g_{k j}\left(\xi^{\prime}, \lambda\right)\right) \varphi_{\infty}\left(\xi^{\prime}, \lambda\right) A^{-2}\left(i \xi_{\ell}\right) \mathscr{F}^{\prime}\left[\partial_{\ell} g_{j}\right]\left(\xi^{\prime}, y_{N}\right)\right\} A e^{-A\left(x_{N}+y_{N}\right)}\right]\left(x^{\prime}\right) d y_{N} .
\end{aligned}
$$

Let $\mathcal{U}_{6}(\lambda)\left(G, G^{\prime}, g_{1}^{\prime \prime}\right)=T_{6}(\lambda) g$. Since the multipliers

$$
\left(\sum_{k=1}^{3} g_{k 1}\left(\xi^{\prime}, \lambda\right)\right) \varphi_{\infty}\left(\xi^{\prime}, \lambda\right) A^{-2}, \quad\left(\sum_{k=1}^{3} g_{k j}\left(\xi^{\prime}, \lambda\right)\right) \varphi_{\infty}\left(\xi^{\prime}, \lambda\right) A^{-2}\left(i \xi_{\ell}\right)
$$

$(j=2,3, \quad \ell=1, \ldots, N-1)$ belong to $\mathbb{M}_{-4,2}\left(\Sigma_{\vartheta}\right)$ as follows from Theorem 3.4 and (3-21), by Lemma 3.6 we have

$$
\mathcal{R}_{\mathcal{L}\left(\mathcal{X}_{q}^{\prime}\left(\mathbb{R}_{+}^{N}\right), L_{q}\left(\mathbb{R}_{+}^{N}\right)\right)}\left(\left\{\left(\tau \partial_{\tau}\right)^{s}\left(\lambda^{j / 2} \partial_{x}^{\alpha} \mathcal{U}_{6}(\lambda)\right) \mid \lambda \in \Sigma_{\vartheta}\right\}\right) \leq C_{N, q} \quad(s=0,1)
$$

for any $j \in \mathbb{N}_{0}$ and $\alpha \in \mathbb{N}_{0}^{N}$ with $j+|\alpha|=4$. Thus, setting $\mathcal{V}_{1}(\lambda)\left(G, G^{\prime}, g_{1}^{\prime \prime}\right)=\sum_{\ell=1}^{6} \mathcal{U}_{\ell}(\lambda)\left(G, G^{\prime}, g_{1}^{\prime \prime}\right)$ and using (3-25)-(3-31), we see that $u(x, \lambda)=\mathcal{V}_{1}(\lambda)\left(g, \lambda^{1 / 2} g, \lambda g\right)$ and that

$$
\mathcal{R}_{\mathcal{L}\left(\mathcal{X}_{q}^{\prime}\left(\mathbb{R}_{+}^{N}\right), L_{q}\left(\mathbb{R}_{+}^{N}\right)\right)}\left(\left\{\left(\tau \partial_{\tau}\right)^{s}\left(\lambda^{j / 2} \partial_{x}^{\alpha} \mathcal{V}_{1}(\lambda)\right) \mid \lambda \in \Sigma_{\vartheta}\right\}\right) \leq C_{N, q} \quad(s=0,1)
$$

for any $j \in \mathbb{N}_{0}$ and $\alpha \in \mathbb{N}_{0}^{N}$ with $j+|\alpha|=4$.

Next, we consider $\theta(x, \lambda)=\mathscr{F}^{\prime-1}\left[\tau\left(\xi^{\prime}, x_{N}, \lambda\right)\right]\left(x^{\prime}\right)$. Applying the Volevich trick and using the identities (3-27) and $A_{k}\left(\xi^{\prime}, \lambda\right)^{4}=\gamma_{k}^{-2} \lambda^{2}+2 \gamma_{k}^{-1} \lambda A^{2}+A^{4}$, we have

$$
\begin{aligned}
& \theta(x, \lambda)=-\lambda \sum_{k, j=1}^{3}\left(\gamma_{k}^{2}+2\right) \mathscr{F}^{\prime-1}\left[g_{k j}\left(\xi^{\prime}, \lambda\right) e^{-A_{k}\left(\xi^{\prime}, \lambda\right) x_{N}} \mathscr{F}\left[g_{j}\right]\left(\xi^{\prime}, 0\right)\right]\left(x^{\prime}\right) \\
& =\sum_{k, j=1}^{3}\left(\gamma_{k}^{2}+2\right) \int_{0}^{\infty} \mathscr{F}^{\prime-1}\left[e^{-A_{k}\left(\xi^{\prime}, \lambda\right)\left(x_{N}+y_{N}\right)} \lambda g_{k j}\left(\xi^{\prime}, \lambda\right) \mathscr{F}\left[\partial_{N} g_{j}\right]\left(\xi^{\prime}, y_{N}\right)\right]\left(x^{\prime}\right) d y_{N} \\
& -\sum_{k, j=1}^{3}\left(\gamma_{k}^{2}+2\right) \int_{0}^{\infty} \mathscr{F}^{\prime-1}\left[e^{-A_{k}\left(\xi^{\prime}, \lambda\right)\left(x_{N}+y_{N}\right)} \lambda A_{k}\left(\xi^{\prime}, \lambda\right) g_{k j}\left(\xi^{\prime}, \lambda\right) \mathscr{F}\left[g_{j}\right]\left(\xi^{\prime}, y_{N}\right)\right]\left(x^{\prime}\right) d y_{N}
\end{aligned}
$$

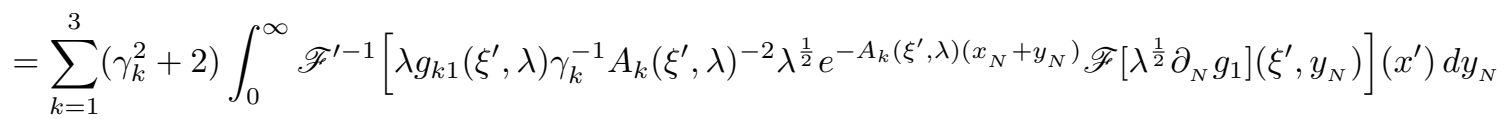

$$
\begin{aligned}
& -\sum_{k=1}^{3} \sum_{\ell=1}^{N-1}\left(\gamma_{k}^{2}+2\right) \int_{0}^{\infty} \mathscr{F}^{\prime-1}\left[\lambda g_{k 1}\left(\xi^{\prime}, \lambda\right) A_{k}\left(\xi^{\prime}, \lambda\right)^{-2}\left(i \xi_{\ell}\right) A^{-1}\right.
\end{aligned}
$$




$$
\begin{aligned}
& \left.A e^{-A_{k}\left(\xi^{\prime}, \lambda\right)\left(x_{N}+y_{N}\right)} \mathscr{F}\left[\partial_{\ell} \partial_{N} g_{1}\right]\left(\xi^{\prime}, y_{N}\right)\right]\left(x^{\prime}\right) d y_{N} \\
& +\sum_{j=2}^{3} \sum_{k=1}^{3}\left(\gamma_{k}^{2}+2\right) \int_{0}^{\infty} \mathscr{F}^{-1}\left[\lambda g_{k j}\left(\xi^{\prime}, \lambda\right) \gamma_{k}^{-1} A_{k}\left(\xi^{\prime}, \lambda\right)^{-2} \lambda^{\frac{1}{2}}\right.
\end{aligned}
$$

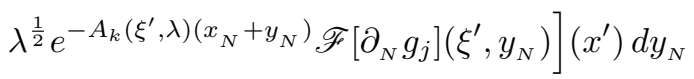

$$
\begin{aligned}
& +\sum_{j=2}^{3} \sum_{k=1}^{3}\left(\gamma_{k}^{2}+2\right) \int_{0}^{\infty} \mathscr{F}^{\prime-1}\left[\lambda g_{k j}\left(\xi^{\prime}, \lambda\right) A_{k}\left(\xi^{\prime}, \lambda\right)^{-2} A A e^{-A_{k}\left(\xi^{\prime}, \lambda\right)\left(x_{N}+y_{N}\right)} \mathscr{F}\left[\partial_{N} g_{j}\right]\left(\xi^{\prime}, y_{N}\right)\right]\left(x^{\prime}\right) d y_{N}
\end{aligned}
$$

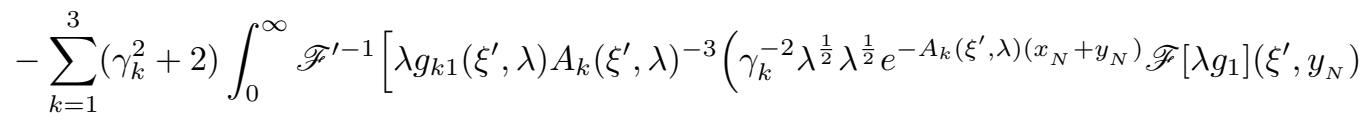

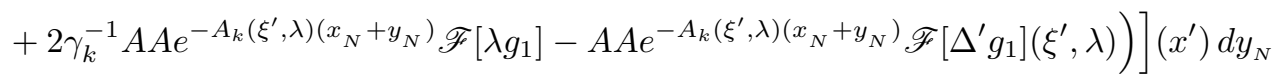

$$
\begin{aligned}
& -\sum_{j=2}^{3} \sum_{k=1}^{3}\left(\gamma_{k}^{2}+2\right) \int_{0}^{\infty} \mathscr{F}^{\prime-1}\left[\lambda g _ { k j } ( \xi ^ { \prime } , \lambda ) A _ { k } ( \xi ^ { \prime } , \lambda ) ^ { - 1 } \left(\gamma_{k}^{-1} \lambda^{\frac{1}{2}} \lambda^{\frac{1}{2}} e^{-A_{k}\left(\xi^{\prime}, \lambda\right)\left(x_{N}+y_{N}\right)} \mathscr{F}\left[\lambda^{\frac{1}{2}} g_{j}\right]\left(\xi^{\prime}, y_{N}\right)\right.\right. \\
& \left.\left.-\sum_{\ell=1}^{N-1}\left(i \xi_{\ell}\right) A^{-1} A e^{-A_{k}\left(\xi^{\prime}, \lambda\right)\left(x_{N}+y_{N}\right)} \mathscr{F}^{\prime}\left[\partial_{\ell} g_{j}\right]\left(\xi^{\prime}, y_{N}\right)\right)\right]\left(x^{\prime}\right) d y_{N}
\end{aligned}
$$

According to the formula above, we define an operator $\mathcal{V}_{2}(\lambda)$ acting on $\left(G, G^{\prime}, g_{1}^{\prime \prime}\right)$ by

$$
\begin{aligned}
& \mathcal{V}_{2}(\lambda)\left(G, G^{\prime}, g_{1}^{\prime \prime}\right)
\end{aligned}
$$

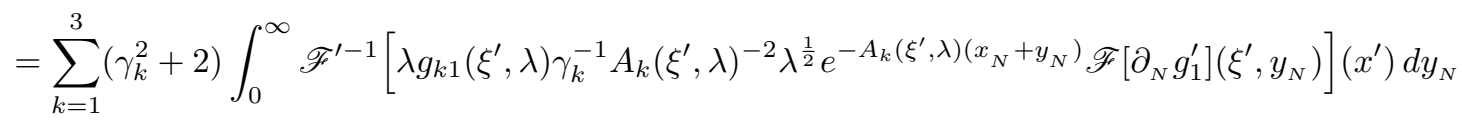

$$
\begin{aligned}
& -\sum_{k=1}^{3} \sum_{\ell=1}^{N-1}\left(\gamma_{k}^{2}+2\right) \int_{0}^{\infty} \mathscr{F}^{\prime-1}\left[\lambda g_{k 1}\left(\xi^{\prime}, \lambda\right) A_{k}\left(\xi^{\prime}, \lambda\right)^{-2}\left(i \xi_{\ell}\right) A^{-1}\right. \\
& \left.A e^{-A_{k}\left(\xi^{\prime}, \lambda\right)\left(x_{N}+y_{N}\right)} \mathscr{F}\left[\partial_{\ell} \partial_{N} g_{1}\right]\left(\xi^{\prime}, y_{N}\right)\right]\left(x^{\prime}\right) d y_{N} \\
& +\sum_{j=2}^{3} \sum_{k=1}^{3}\left(\gamma_{k}^{2}+2\right) \int_{0}^{\infty} \mathscr{F}^{\prime-1}\left[\lambda g_{k j}\left(\xi^{\prime}, \lambda\right) \gamma_{k}^{-1} A_{k}\left(\xi^{\prime}, \lambda\right)^{-2} \lambda^{\frac{1}{2}}\right.
\end{aligned}
$$

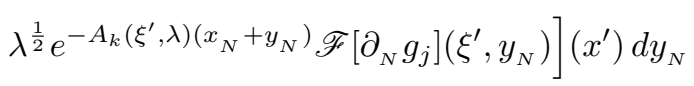

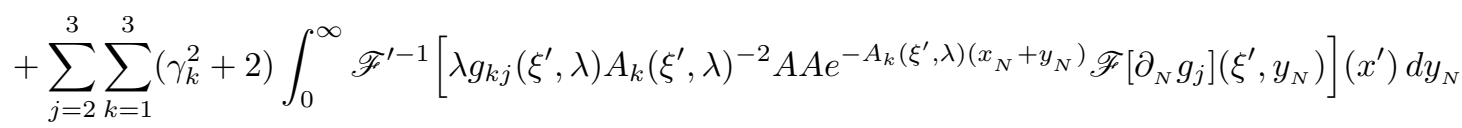

$$
\begin{aligned}
& -\sum_{k=1}^{3}\left(\gamma_{k}^{2}+2\right) \int_{0}^{\infty} \mathscr{F}^{\prime-1}\left[\lambda g _ { k 1 } ( \xi ^ { \prime } , \lambda ) A _ { k } ( \xi ^ { \prime } , \lambda ) ^ { - 3 } \left(\gamma_{k}^{-2} \lambda^{\frac{1}{2}} \lambda^{\frac{1}{2}} e^{-A_{k}\left(\xi^{\prime}, \lambda\right)\left(x_{N}+y_{N}\right)} \mathscr{F}\left[g_{1}^{\prime \prime}\right]\left(\xi^{\prime}, y_{N}\right)\right.\right. \\
& \left.\left.+2 \gamma_{k}^{-1} A A e^{-A_{k}\left(\xi^{\prime}, \lambda\right)\left(x_{N}+y_{N}\right)} \mathscr{F}\left[g_{1}^{\prime \prime}\right]-A A e^{-A_{k}\left(\xi^{\prime}, \lambda\right)\left(x_{N}+y_{N}\right)} \mathscr{F}\left[\Delta^{\prime} g_{1}\right]\left(\xi^{\prime}, \lambda\right)\right)\right]\left(x^{\prime}\right) d y_{N}
\end{aligned}
$$

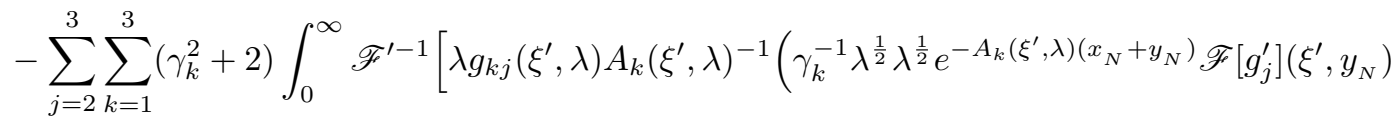

$$
\begin{aligned}
& \left.\left.-\sum_{\ell=1}^{N-1}\left(i \xi_{\ell}\right) A^{-1} A e^{-A_{k}\left(\xi^{\prime}, \lambda\right)\left(x_{N}+y_{N}\right)} \mathscr{F}^{\prime}\left[\partial_{\ell} g_{j}\right]\left(\xi^{\prime}, y_{N}\right)\right)\right]\left(x^{\prime}\right) d y_{N} .
\end{aligned}
$$


With this definition, we obtain $\mathcal{V}_{2}(\lambda)\left(g, \lambda^{1 / 2} g, \lambda g_{1}\right)=\theta$. Moreover, by Theorem 3.4 we see that the multipliers

$$
\lambda g_{k 1}\left(\xi^{\prime}, \lambda\right) A_{k}\left(\xi^{\prime}, \lambda\right)^{-2}, \lambda g_{k j}\left(\xi^{\prime}, \lambda\right) A_{k}\left(\xi^{\prime}, \lambda\right)^{-2} \lambda^{1 / 2}, \lambda g_{k 1}\left(\xi^{\prime}, \lambda\right) A_{k}\left(\xi^{\prime}, \lambda\right)^{-3} \lambda^{1 / 2},
$$

belong to $\mathbb{M}_{-2,1}\left(\Sigma_{\vartheta}\right)$ and that the multipliers

$$
\begin{aligned}
& \lambda g_{k 1}\left(\xi^{\prime}, \lambda\right) A_{k}\left(\xi^{\prime}, \lambda\right)^{-2}\left(i \xi_{\ell}\right) A^{-1}, \quad \lambda g_{k j}\left(\xi^{\prime}, \lambda\right) A_{k}\left(\xi^{\prime}, \lambda\right)^{-2} A, \\
& \lambda g_{k 1}\left(\xi^{\prime}, \lambda\right) A_{k}\left(\xi^{\prime}, \lambda\right)^{-3} A, \quad \lambda g_{k j}\left(\xi^{\prime}, \lambda\right) A_{k}\left(\xi^{\prime}, \lambda\right)^{-1}\left(i \xi_{\ell}\right) A^{-1}
\end{aligned}
$$

belong to $\mathbb{M}_{-2,2}\left(\Sigma_{\vartheta}\right)$. Thus, by Lemma 3.5 , we have

$$
\mathcal{R}_{\mathcal{L}\left(\mathcal{X}_{q}^{\prime}\left(\mathbb{R}_{+}^{N}\right), L_{q}\left(\mathbb{R}_{+}^{N}\right)\right)}\left(\left\{\left(\tau \partial_{\tau}\right)^{s}\left(\lambda^{j / 2} \partial_{x}^{\alpha} \mathcal{V}_{2}(\lambda)\right) \mid \lambda \in \Sigma_{\vartheta}\right\}\right) \leq C_{N, q} \quad(s=0,1)
$$

for any $j \in \mathbb{N}_{0}$ and $\alpha \in \mathbb{N}_{0}^{N}$ with $j+|\alpha|=2$.

Let $\mathcal{V}_{1}(\lambda)=\sum_{\ell=1}^{6} \mathcal{U}_{\ell}(\lambda)$ and $\mathcal{V}(\lambda)=\left(\mathcal{V}_{1}(\lambda), \mathcal{V}_{2}(\lambda)\right)^{\top}$. Then

$$
U=\left(\mathcal{V}_{1}(\lambda)\left(g, \lambda^{1 / 2} g, \lambda g_{1}\right), \lambda \mathcal{V}_{1}(\lambda)\left(g, \lambda^{1 / 2} g, \lambda g_{1}\right), \mathcal{V}_{2}(\lambda)\left(g, \lambda^{1 / 2} g, \lambda g_{1}\right)\right)^{\top}
$$

satisfies the equations (3-1) with $F=0$. And, by Proposition 2.2 c), (3-32), and (3-33), we have

$$
\begin{aligned}
& \mathcal{R}_{\mathcal{L}\left(\mathcal{X}_{q}^{\prime}\left(\mathbb{R}_{+}^{N}\right), H_{q}^{4-j}\left(\mathbb{R}_{+}^{N}\right)\right)}\left(\left\{\left(\tau \partial_{\tau}\right)^{s}\left(\lambda^{j / 2} \mathcal{V}_{1}(\lambda)\right) \mid \lambda \in \Sigma_{\vartheta, \lambda_{0}}\right\}\right) \leq C_{N, q, \lambda_{0}} \quad(j=0,1,2,3,4), \\
& \mathcal{R}_{\mathcal{L}\left(\mathcal{X}_{q}^{\prime}\left(\mathbb{R}_{+}^{N}\right), H_{q}^{2-j}\left(\mathbb{R}_{+}^{N}\right)\right)}\left(\left\{\left(\tau \partial_{\tau}\right)^{s}\left(\lambda^{j / 2} \mathcal{V}_{k}(\lambda)\right) \mid \lambda \in \Sigma_{\vartheta, \lambda_{0}}\right\}\right) \leq C_{N, q, \lambda_{0}} \quad(j=0,1,2, \quad k=2,3),
\end{aligned}
$$

for any $\lambda_{0}>0$ and $s=0,1$.

To define $\mathcal{T}(\lambda)$ required in Theorem 3.1, we want to apply $\mathcal{V}(\lambda)$ to $g-B(D) \mathcal{S}_{+}(\lambda) F^{\prime}$ with $\mathcal{S}_{+}(\lambda)=$ $\left(\mathcal{S}_{+1}(\lambda), \lambda \mathcal{S}_{+1}(\lambda), \mathcal{S}_{+2}(\lambda)\right)^{\top}$ where $\mathcal{S}_{+1}(\lambda)$ and $\mathcal{S}_{+2}(\lambda)$ are defined in (3-4). Observe that

$$
\left.B(D) \tilde{\mathcal{S}}_{+}(\lambda) F^{\prime}=\left(\Delta-(1-\beta) \Delta^{\prime}\right) \mathcal{S}_{+1}(\lambda) F^{\prime}+\mathcal{S}_{+2}(\lambda) F^{\prime}, \partial_{N}\left(\Delta+(1-\beta) \Delta^{\prime}\right) \mathcal{S}_{+1}(\lambda) F^{\prime}, \partial_{N} \mathcal{S}_{+2}(\lambda) F^{\prime}\right)^{\top} .
$$

Thus, we define $\mathcal{T}(\lambda)$ acting on $\left(F^{\prime}, G, G^{\prime}, g_{1}^{\prime \prime}\right)$ by

$$
\mathcal{T}(\lambda)\left(F^{\prime}, G, G^{\prime}, g_{1}^{\prime \prime}\right)=\mathcal{S}_{+}(\lambda) F^{\prime}+\mathcal{V}(\lambda)\left(G, G^{\prime}, g_{1}^{\prime \prime}\right)-\mathcal{V}(\lambda)\left(B(D) \mathcal{S}_{+}(\lambda) F^{\prime}, \lambda^{1 / 2} B(D) \mathcal{S}_{+}(\lambda) F^{\prime}, \mathcal{G}_{3}\right)
$$

with $\mathcal{G}_{3}=\left(\Delta-(1-\beta) \Delta^{\prime}\right) \mathcal{S}_{+1}(\lambda) F^{\prime}+\mathcal{S}_{+2}(\lambda) F^{\prime}$. By $(3-5)$,

$$
\begin{aligned}
& \left.\mathcal{R}_{\mathcal{L}\left(L_{q}\left(\mathbb{R}_{+}^{N}\right)^{2}, H^{4-j}\left(\mathbb{R}_{+}^{N}\right)\right)}\left\{\left(\tau \partial_{t}\right)^{s}\left(\lambda^{j / 2} \mathcal{S}_{+1}(\lambda)\right) \mid \lambda \in \Sigma_{\vartheta_{0}, \lambda_{0}}\right\}\right) \leq C_{\lambda_{0}} \quad(s=0,1, \quad j=0,1,2,3,4), \\
& \left.\mathcal{R}_{\mathcal{L}\left(L_{q}\left(\mathbb{R}_{+}^{N}\right)^{2}, H^{2-j}\left(\mathbb{R}_{+}^{N}\right)\right)}\left\{\left(\tau \partial_{t}\right)^{s}\left(\lambda^{j / 2} \mathcal{S}_{+2}(\lambda)\right) \mid \lambda \in \Sigma_{\vartheta_{0}, \lambda_{0}}\right\}\right) \leq C_{\lambda_{0}} \quad(s=0,1, \quad j=0,1,2),
\end{aligned}
$$

and therefore, by (3-34), (3-35) and Proposition 2.2, we obtain Theorem 3.1.

\section{Proof of Theorem 3.4}

The purpose of this section is to prove Theorem 3.4. For this, we start with the uniqueness of the solution of the system of ordinary differential equations:

$$
\begin{array}{cc}
\lambda^{2} w+\left(\partial_{N}^{2}-\left|\xi^{\prime}\right|^{2}\right)^{2} w+\left(\partial_{N}^{2}-\left|\xi^{\prime}\right|^{2}\right) \tau=0 & \left(x_{N}>0\right), \\
\lambda \tau-\left(\partial_{N}^{2}-\left|\xi^{\prime}\right|^{2}\right) \tau-\lambda\left(\partial_{N}^{2}-\left|\xi^{\prime}\right|^{2}\right) w=0 & \left(x_{N}>0\right)
\end{array}
$$

with boundary conditions

$$
\begin{aligned}
\left(\partial_{N}^{2}-\left|\xi^{\prime}\right|^{2}\right) w(0)+(1-\beta)\left|\xi^{\prime}\right|^{2} w(0)+\tau(0) & =0, \\
\partial_{N}\left(\left(\partial_{N}^{2}-\left|\xi^{\prime}\right|^{2}\right) w(0)-(1-\beta)\left|\xi^{\prime}\right|^{2} w(0)\right) & =0, \\
\partial_{N} \tau(0) & =0 .
\end{aligned}
$$

Let $w$ and $\tau$ be $C^{\infty}$-functions defined on $(0, \infty)$. If $w$ and $\tau$ satisfy equations (4-1) and (4-2) and the condition: $\left|w\left(x_{N}\right)\right|+\mid \tau\left(x_{N} \mid \leq C e^{-c x_{N}}\right.$ with some positive constants $C$ and $c$, then $w$ and $\tau$ are called stable solutions. 
Lemma 4.1. Let $\lambda \in \mathbb{C} \backslash\{0\}$ with $\operatorname{Re} \lambda \geq 0$ and $\xi^{\prime} \in \mathbb{R}^{N-1}$. If $w$ and $\tau$ be stable solutions of problem (4-1) and (4-2), then $w=\tau=0$.

Proof. Following [24, proof of Lemma 4.1], we multiply the first equation in (4-1) by $\bar{\lambda} \bar{w}$, the second by $\bar{\tau}$, take the sum and integrate over $(0, \infty)$. We get

$$
\begin{aligned}
S & :=\lambda|\lambda|^{2}\|w\|^{2}+\bar{\lambda}\left\langle\left(\partial_{N}^{2}-\left|\xi^{\prime}\right|^{2}\right)^{2} w, w\right\rangle+\bar{\lambda}\left\langle\left(\partial_{N}^{2}-\left|\xi^{\prime}\right|^{2}\right) \tau, w\right\rangle+\lambda\|\tau\|^{2} \\
& -\left\langle\left(\partial_{N}^{2}-\left|\xi^{\prime}\right|^{2}\right) \tau, \tau\right\rangle-\lambda\left\langle\left(\partial_{N}^{2}-\left|\xi^{\prime}\right|^{2}\right) w, \tau\right\rangle=0 .
\end{aligned}
$$

Here $\langle\cdot, \cdot\rangle$ is the scalar product in $L_{2}((0, \infty))$ and $\|\cdot\|$ the norm in $L_{2}((0, \infty))$. First we show that for all $w$ satisfying the boundary conditions (4-2), we have

$$
\left\langle\left(\partial_{N}^{2}-\left|\xi^{\prime}\right|^{2}\right)^{2} w, w\right\rangle=s-\tau(0) \partial_{N} \bar{w}(0)
$$

with a real and non-negative number $s$. For this we write by integration by parts

$$
\begin{aligned}
\left\langle\left(\partial_{N}^{2}-\left|\xi^{\prime}\right|^{2}\right)^{2} w, w\right\rangle & =\left\|\partial_{N}^{2} w\right\|^{2}+2\left|\xi^{\prime}\right|^{2}\left\|\partial_{N} w\right\|^{2}+\left|\xi^{\prime}\right|^{4}\|w\|^{2} \\
& -\partial_{N}^{3} w(0) \bar{w}(0)+\partial_{N}^{2} w(0) \partial_{N} \bar{w}(0)+2\left|\xi^{\prime}\right|^{2} \partial_{N} w(0) \bar{w}(0) .
\end{aligned}
$$

Inserting the boundary conditions (4-2) yields that

$$
\begin{aligned}
& \left\langle\left(\partial_{N}^{2}-\left|\xi^{\prime}\right|^{2}\right)^{2} w, w\right\rangle=\left\|\partial_{N}^{2} w\right\|^{2}+2\left|\xi^{\prime}\right|^{2}\left\|\partial_{N} w\right\|^{2}+\left|\xi^{\prime}\right|^{4}\|w\|^{2} \\
& \quad-(2-\beta)\left|\xi^{\prime}\right|^{2} \partial_{N} w(0) \bar{w}(0)+\left(\beta\left|\xi^{\prime}\right|^{2} w(0)-\tau(0)\right) \partial_{N} \bar{w}(0)+2\left|\xi^{\prime}\right|^{2} \partial_{N} w(0) \bar{w}(0) \\
& \quad=s-\tau(0) \partial_{N} \bar{w}(0)
\end{aligned}
$$

with $s:=\left\|\partial_{N}^{2} w\right\|^{2}+2\left|\xi^{\prime}\right|^{2}\left\|\partial_{N} w\right\|^{2}+\left|\xi^{\prime}\right|^{4}\|w\|^{2}+2 \beta\left|\xi^{\prime}\right|^{2} \operatorname{Re}\left(w(0) \partial_{N} \bar{w}(0)\right)$. Since

$$
\begin{aligned}
& \left|\operatorname{Re}\left(w(0) \partial_{N} \bar{w}(0)\right)\right| \leq\left|w(0) \partial_{N} \bar{w}(0)\right|=\left|\int_{0}^{\infty} \partial_{N}\left[w\left(x_{N}\right) \partial_{N} \bar{w}\left(x_{N}\right)\right] d x_{N}\right| \\
& \quad \leq \int_{0}^{\infty}\left[\left|\partial_{N} w\left(x_{N}\right)\right|^{2}+\left|w\left(x_{N}\right) \partial_{N}^{2} \bar{w}\left(x_{N}\right)\right|\right] d x_{N} \leq\left\|\partial_{N} w\right\|^{2}+\|w\| \cdot\left\|\partial_{N}^{2} w\right\|
\end{aligned}
$$

noting that $\beta<1$, we have

$$
s \geq\left\|\partial_{N}^{2} w\right\|^{2}+\left|\xi^{\prime}\right|^{4}\|w\|^{2}-2\left|\xi^{\prime}\right|^{2}\|w\|\left\|\partial_{N}^{2} w\right\| \geq 0 .
$$

With integration by parts again, we have

$$
\begin{aligned}
S & =\lambda|\lambda|^{2}\|w\|^{2}+\bar{\lambda} s-\bar{\lambda} \tau(0) \partial_{N} \bar{w}(0) \\
& -\bar{\lambda}\left\langle\partial_{N} \tau, \partial_{N} w\right\rangle-\bar{\lambda} \partial_{N} \tau(0) \bar{w}(0)-\bar{\lambda}\left|\xi^{\prime}\right|^{2}\langle\tau, w\rangle+\lambda\|\tau\|^{2}+\left\|\partial_{N} \tau\right\|^{2}+\partial_{N} \tau(0) \bar{\tau}(0) \\
& +\left|\xi^{\prime}\right|^{2}\|\tau\|^{2}+\lambda\left\langle\partial_{N} w, \partial_{N} \tau\right\rangle+\lambda \partial_{N} w(0) \bar{\tau}(0)+\lambda\left|\xi^{\prime}\right|^{2}\langle w, \tau\rangle=0 .
\end{aligned}
$$

Taking the real part and noting that $\partial_{N} \tau(0)=0$ yield that

$$
0=\operatorname{Re} S=\operatorname{Re} \lambda\left[|\lambda|^{2}\|w\|^{2}+s+\|\tau\|^{2}\right]+\left\|\partial_{N} \tau\right\|^{2}+\left|\xi^{\prime}\right|^{2}\|\tau\|^{2} .
$$

As $\operatorname{Re} \lambda \geq 0$ and $s \geq 0$, we obtain $\left\|\partial_{N} \tau\right\|=0$. Therefore, $\tau$ is a constant and from $\tau\left(x_{N}\right) \rightarrow 0 \quad\left(x_{N} \rightarrow \infty\right)$ we get $\tau=0$. Inserting this into the second equation in (4-1) and noting $\lambda \neq 0$ yield that $\left(\partial_{N}^{2}-\left|\xi^{\prime}\right|^{2}\right) w=$ $0 \quad\left(x_{N}>0\right)$. From the first equation in (4-1) we now get $\lambda^{2} w=0$ and thus $w=0$.

In what follows, let $\Delta\left(\xi^{\prime}, \lambda\right)$ be the Lopatinskiu matrix defined in (3-12). To prove Theorem 3.4, one of the tasks is to analyze the determinant of $\Delta\left(\xi^{\prime}, \lambda\right)$. The determinant is given by

$$
\begin{aligned}
\operatorname{det} \Delta\left(\xi^{\prime}, \lambda\right) & =\lambda\left(-\gamma_{1} \lambda+\zeta\right) A_{2} A_{3}\left[\left(-\frac{\lambda}{\gamma_{2}}+\zeta\right)\left(\gamma_{3}^{2}+2\right)-\left(-\frac{\lambda}{\gamma_{3}}+\zeta\right)\left(\gamma_{2}^{2}+2\right)\right] \\
& +\lambda\left(-\gamma_{2} \lambda+\zeta\right) A_{3} A_{1}\left[\left(-\frac{\lambda}{\gamma_{3}}+\zeta\right)\left(\gamma_{1}^{2}+2\right)-\left(-\frac{\lambda}{\gamma_{1}}+\zeta\right)\left(\gamma_{3}^{2}+2\right)\right]
\end{aligned}
$$




$$
+\lambda\left(-\gamma_{3} \lambda+\zeta\right) A_{1} A_{2}\left[\left(-\frac{\lambda}{\gamma_{1}}+\zeta\right)\left(\gamma_{2}^{2}+2\right)-\left(-\frac{\lambda}{\gamma_{2}}+\zeta\right)\left(\gamma_{1}^{2}+2\right)\right]
$$

with $\zeta:=(1-\beta)\left|\xi^{\prime}\right|^{2}$. A simple calculation shows that for $i \neq j$ we have

$$
\left(-\frac{\lambda}{\gamma_{i}}+\zeta\right)\left(\gamma_{j}^{2}+2\right)-\left(-\frac{\lambda}{\gamma_{j}}+\zeta\right)\left(\gamma_{i}^{2}+2\right)=\left(\gamma_{i}^{2}-\gamma_{j}^{2}\right)\left(\frac{\lambda}{\gamma_{i} \gamma_{j}}-\zeta\right) .
$$

Moreover, recalling that $\gamma_{1} \gamma_{2} \gamma_{3}=1$ we have $\frac{\lambda}{\gamma_{2} \gamma_{3}}-\zeta=\gamma_{1} \lambda-\zeta$. Therefore, we get

$$
\operatorname{det} \Delta\left(\xi^{\prime}, \lambda\right)=\lambda\left\{\left(\gamma_{3}^{2}-\gamma_{2}^{2}\right) A_{2} A_{3}\left(\gamma_{1} \lambda-\zeta\right)^{2}+\left(\gamma_{1}^{2}-\gamma_{3}^{2}\right) A_{3} A_{1}\left(\gamma_{2} \lambda-\zeta\right)^{2}+\left(\gamma_{2}^{2}-\gamma_{1}^{2}\right) A_{1} A_{2}\left(\gamma_{3} \lambda-\zeta\right)^{2}\right\} \text {. }
$$

(i) Firstly, we estimate $\operatorname{det} \Delta\left(\xi^{\prime}, \lambda\right)$ for $\left(\xi^{\prime}, \lambda\right) \in \mathbb{R}^{N-1} \times \Sigma_{\vartheta_{0}}$ with $|\lambda| \leq \sigma_{0}\left|\xi^{\prime}\right|^{2}$, where $\vartheta_{0}$ is the number given in (2-6) and $\sigma_{0}$ is a small positive number chosen later. We derive an asymptotic expansion of $\operatorname{det} \Delta\left(\xi^{\prime}, \lambda\right)$ in $t:=\frac{\lambda}{|\xi|^{2}}$ for $t \rightarrow 0$. In what follows, the order $O\left(t^{\ell}\right)(\ell=1,2)$ means that there exists a $C^{\infty}$-function $g(t)$ defined on $|t| \leq \sigma_{0}$ such that $O\left(t^{\ell}\right)$ is represented by $O\left(t^{\ell}\right)=t^{\ell} g(t)$. Let $f(t)=t^{\ell} g(t)$, and then by the Bell formula we have

$$
\left|\partial_{\xi^{\prime}}^{\alpha^{\prime}} f\left(\lambda /\left|\xi^{\prime}\right|^{2}\right)\right| \leq C_{f, \alpha^{\prime}}\left(|\lambda| /\left|\xi^{\prime}\right|^{2}\right)^{\ell}\left|\xi^{\prime}\right|^{-\left|\alpha^{\prime}\right|}
$$

for any $\alpha^{\prime} \in \mathbb{N}_{0}^{N-1}$ whenever $|\lambda|^{1 / 2} /\left|\xi^{\prime}\right| \leq \sigma_{0}$. In fact, we have

$$
\begin{aligned}
\left|\partial_{\xi^{\prime}}^{\alpha^{\prime}} f\left(\lambda /\left|\xi^{\prime}\right|^{2}\right)\right| & \leq C_{\alpha^{\prime}} \sum_{m=1}^{\left|\alpha^{\prime}\right|}\left|f^{(m)}\left(\lambda /\left|\xi^{\prime}\right|^{2}\right)\right| \sum_{\substack{\alpha_{1}^{\prime}+\cdots+\alpha_{m}^{\prime}=\alpha^{\prime} \\
\left|\alpha_{k}^{\prime}\right| \geq 1}}\left|\partial_{\xi^{\prime}}^{\alpha_{1}^{\prime}}\left(\lambda /\left|\xi^{\prime}\right|^{2}\right)\right| \cdots\left|\partial_{\xi^{\prime}}^{\alpha_{m}^{\prime}}\left(\lambda /\left|\xi^{\prime}\right|^{2}\right)\right| \\
& \leq C_{f, \alpha^{\prime}}\left(\left.|\lambda /| \xi^{\prime}\right|^{2}\right)^{\ell}\left|\xi^{\prime}\right|^{-\left|\alpha^{\prime}\right|}
\end{aligned}
$$

provided that $|\lambda|^{1 / 2} /\left|\xi^{\prime}\right| \leq \sigma_{0}(\leq 1)$.

Since $\sigma_{0}$ will be chosen as a small positive number eventually, we may assume that $\sigma_{0}\left|\gamma_{j}\right|^{-1} \leq 1 / 2$ for $j=1,2,3$. Let $t=\lambda /\left|\xi^{\prime}\right|^{2}$ and $|t| \leq \sigma_{0} / 2$. By the Taylor formula, we have $A_{j}=\sqrt{\gamma_{j}^{-1} \lambda+\left|\xi^{\prime}\right|^{2}}=$ $\left|\xi^{\prime}\right|\left(1+\left(2 \gamma_{j}\right)^{-1} t+O\left(t^{2}\right)\right)$. Since

$$
\gamma_{j} \lambda-\zeta=\gamma_{j} \lambda-(1-\beta)\left|\xi^{\prime}\right|^{2}=\left|\xi^{\prime}\right|^{2}\left(\gamma_{j} t-(1-\beta)\right),
$$

we obtain

$$
\begin{aligned}
\operatorname{det} \Delta\left(\xi^{\prime}, \lambda\right) & =\lambda\left|\xi^{\prime}\right|^{6}\left\{\left(\gamma_{3}^{2}-\gamma_{2}^{2}\right)\left(1+\frac{t}{2 \gamma_{2}}+O\left(t^{2}\right)\right)\left(1+\frac{t}{2 \gamma_{3}}+O\left(t^{2}\right)\right)\left(\gamma_{1} t-(1-\beta)\right)^{2}\right. \\
& +\left(\gamma_{1}^{2}-\gamma_{3}^{2}\right)+O\left(t^{2}\right)\left(1+\frac{t}{2 \gamma_{3}}+O\left(t^{2}\right)\right)\left(1+\frac{t}{2 \gamma_{1}}+O\left(t^{2}\right)\right)\left(\gamma_{2} t-(1-\beta)\right)^{2} \\
& \left.+\left(\gamma_{2}^{2}-\gamma_{1}^{2}\right)\left(1+\frac{t}{2 \gamma_{1}}+O\left(t^{2}\right)\right)\left(1+\frac{t}{2 \gamma_{2}}+O\left(t^{2}\right)\right)\left(\gamma_{3} t-(1-\beta)\right)^{2}\right\} \\
& =\lambda\left|\xi^{\prime}\right|^{6}\left(c_{0}+c_{1} t+O\left(t^{2}\right)\right), \quad t \rightarrow 0 .
\end{aligned}
$$

Here

$$
c_{0}=(1-\beta)^{2}\left(\left(\gamma_{3}^{2}-\gamma_{2}^{2}\right)+\left(\gamma_{1}^{2}-\gamma_{3}^{2}\right)+\left(\gamma_{2}^{2}-\gamma_{1}^{2}\right)\right)=0
$$

and

$$
c_{1}=\frac{1}{2}(1-\beta)^{2} c_{11}-2(1-\beta) c_{12}
$$

with

$$
\begin{aligned}
& c_{11}=\left(\gamma_{3}^{2}-\gamma_{2}^{2}\right)\left(\frac{1}{\gamma_{2}}+\frac{1}{\gamma_{3}}\right)+\left(\gamma_{1}^{2}-\gamma_{3}^{2}\right)\left(\frac{1}{\gamma_{3}}+\frac{1}{\gamma_{1}}\right)+\left(\gamma_{2}^{2}-\gamma_{1}^{2}\right)\left(\frac{1}{\gamma_{1}}+\frac{1}{\gamma_{2}}\right), \\
& c_{12}=\left(\gamma_{3}^{2}-\gamma_{2}^{2}\right) \gamma_{1}+\left(\gamma_{1}^{2}-\gamma_{3}^{2}\right) \gamma_{2}+\left(\gamma_{2}^{2}-\gamma_{1}^{2}\right) \gamma_{3} .
\end{aligned}
$$

Making use of $\gamma_{j}^{3}=\gamma_{j}^{2}-2 \gamma_{j}+1$ and $\gamma_{1} \gamma_{2} \gamma_{3}=1$, straightforward calculation shows

$$
c_{11}=c_{12}=\left(\gamma_{1}-\gamma_{2}\right)\left(\gamma_{1}-\gamma_{3}\right)\left(\gamma_{2}-\gamma_{3}\right) \text {. }
$$


Consequently,

$$
c_{1}=\left(\gamma_{1}-\gamma_{2}\right)\left(\gamma_{1}-\gamma_{2}\right)\left(\gamma_{2}-\gamma_{3}\right)(1-\beta)\left(-\frac{3}{2}-\frac{\beta}{2}\right) \neq 0
$$

We obtain

$$
\operatorname{det} \Delta\left(\xi^{\prime}, \lambda\right)=\left|\xi^{\prime}\right|^{4} \lambda^{2}\left(c_{1}+O(t)\right) \quad\left(t=\frac{\lambda}{\left|\xi^{\prime}\right|^{2}}\right)
$$

for $|t| \leq \sigma_{0}$ with sufficiently small $\sigma_{0}>0$.

(ii) Secondly, we estimate $\Delta\left(\xi^{\prime}, \lambda\right)$ for $\left(\xi^{\prime}, \lambda\right) \in \mathbb{R}^{N-1} \times \mathbb{C}$ with $\sigma_{0}\left|\xi^{\prime}\right|^{2} \leq|\lambda|$. Let $\tilde{\lambda}=\lambda r^{-2}$ and $\tilde{\xi}=\xi r^{-1}$ with $r=\left(|\lambda|+\left|\xi^{\prime}\right|^{2}\right)^{1 / 2}$, and then, $|\tilde{\lambda}|+\left|\tilde{\xi}^{\prime}\right|^{2}=1$. Note that

$$
\operatorname{det} \Delta\left(r \xi^{\prime}, r^{2} \lambda\right)=r^{8} \operatorname{det} \Delta\left(\tilde{\xi}^{\prime}, \tilde{\lambda}\right) \quad(r>0) .
$$

Let

$$
\Xi=\left\{\left(\tilde{\xi}^{\prime}, \tilde{\lambda}\right) \in \mathbb{R}^{N-1} \times\left.\mathbb{C}|| \tilde{\lambda}|+| \tilde{\xi}^{\prime}\right|^{2}=1, \quad|\tilde{\lambda}| \geq \frac{\sigma_{0}}{1+\sigma_{0}}, \quad\left|\tilde{\xi}^{\prime}\right|^{2} \leq \frac{1}{1+\sigma_{0}}\right\} .
$$

Note that $\left(\tilde{\xi}^{\prime}, \tilde{\lambda}\right) \in \Xi$ for $\left(\xi^{\prime}, \lambda\right) \in \mathbb{R}^{N-1} \times \mathbb{C}$ with $\sigma_{0}\left|\xi^{\prime}\right|^{2} \leq|\lambda|$. By Lemma 4.1, we know that $\operatorname{det} \Delta\left(\tilde{\xi}^{\prime}, \tilde{\lambda}\right) \neq$ 0 for $\left(\tilde{\xi}^{\prime}, \tilde{\lambda}\right) \in \Xi$ with $\operatorname{Re} \tilde{\lambda} \geq 0$. Since $\operatorname{det} \Delta\left(\tilde{\xi}^{\prime}, \tilde{\lambda}\right)$ is a continuous function, there exists a $\vartheta>\pi / 2$ such that $\operatorname{det} \Delta\left(\tilde{\xi}^{\prime}, \tilde{\lambda}\right) \neq 0$ for $\left(\tilde{\xi}^{\prime}, \tilde{\lambda}\right) \in \Xi$ with $\tilde{\lambda} \in \Sigma_{\vartheta}$, which furnishes that

$$
\left|\operatorname{det} \Delta\left(\xi^{\prime}, \lambda\right)\right| \geq c_{2}\left(|\lambda|^{1 / 2}+|\xi|\right)^{8}
$$

with some $c_{2}>0$ provided that $\left(\xi^{\prime}, \lambda\right) \in \mathbb{R}^{N-1} \times \Sigma_{\vartheta}$ and $\sigma_{0}\left|\xi^{\prime}\right|^{2} \leq|\lambda|$.

Next, we consider $\Delta\left(\xi^{\prime}, \lambda\right)^{-1}=\left(g_{i j}\left(\xi^{\prime}, \lambda\right)\right)$. Let

$$
\begin{array}{ll}
\Xi_{\vartheta, \sigma_{0}}=\left\{\left(\xi^{\prime}, \lambda\right) \in \mathbb{R}^{N-1} \times\left.\Sigma_{\vartheta}\left|\sigma_{0}\right| \xi^{\prime}|\geq| \lambda\right|^{1 / 2},\right. & \left.\lambda \in \Sigma_{\vartheta}\right\}, \\
\Xi_{\vartheta, \sigma_{0}}^{\prime}=\left\{\left(\xi^{\prime}, \lambda\right) \in \mathbb{R}^{N-1} \times\left.\Sigma_{\vartheta}\left|\sigma_{0}\right| \xi^{\prime}|\leq| \lambda\right|^{1 / 2},\right. & \left.\lambda \in \Sigma_{\vartheta}\right\} .
\end{array}
$$

(iii) Firstly, we consider $g_{i 1}\left(\xi^{\prime}, \lambda\right)$. The coefficient of $g_{11}$ is given by

$$
\begin{aligned}
g_{11}\left(\xi^{\prime}, \lambda\right) & =\frac{\lambda A_{2} A_{3}}{\operatorname{det} \Delta\left(\xi^{\prime}, \lambda\right)}\left[\left(-\frac{\lambda}{\gamma_{2}}+\zeta\right)\left(\gamma_{3}^{2}+2\right)-\left(-\frac{\lambda}{\gamma_{3}}+\zeta\right)\left(\gamma_{2}^{2}+2\right)\right] \\
& =\frac{\lambda A_{2} A_{3}}{\operatorname{det} \Delta\left(\xi^{\prime}, \lambda\right)}\left[\lambda\left(\frac{\gamma_{2}^{2}}{\gamma_{3}}-\frac{\gamma_{3}^{2}}{\gamma_{2}}+\frac{2}{\gamma_{3}}-\frac{2}{\gamma_{2}}\right)+\zeta\left(\gamma_{3}^{2}-\gamma_{2}^{2}\right)\right] \\
& =\frac{\lambda A_{2} A_{3}}{\operatorname{det} \Delta\left(\xi^{\prime}, \lambda\right)}\left[\lambda \frac{\gamma_{2}^{3}-\gamma_{3}^{3}+2 \gamma_{2}-2 \gamma_{3}}{\gamma_{2} \gamma_{3}}+\zeta\left(\gamma_{3}^{2}-\gamma_{2}^{2}\right)\right] \\
& =\frac{\lambda A_{2} A_{3}}{\operatorname{det} \Delta\left(\xi^{\prime}, \lambda\right)}\left(\gamma_{2}^{2}-\gamma_{3}^{2}\right)\left(\gamma_{1} \lambda-\zeta\right)
\end{aligned}
$$

where we again used $\gamma_{j}^{3}+2 \gamma_{j}=\gamma_{j}^{2}+1$ and $\gamma_{1} \gamma_{2} \gamma_{3}=1$. By (4-4) and the Bell formula, we have

$$
\left|\partial_{\xi^{\prime}}^{\alpha^{\prime}}\left(\operatorname{det} \Delta\left(\xi^{\prime}, \lambda\right)\right)^{-1}\right| \leq C_{\alpha^{\prime}}\left(|\lambda|^{1 / 2}+\left|\xi^{\prime}\right|\right)^{-8-\left|\alpha^{\prime}\right|}
$$

for any $\alpha^{\prime} \in \mathbb{N}_{0}^{N-1}$ and $\left(\xi^{\prime}, \lambda\right) \in \Xi_{\vartheta, \sigma_{0}}^{\prime}$, so that by the Leibniz rule, (3-15), (3-17) with $s=1$, and the permutation of indices, we have

$$
\left|\partial_{\xi^{\prime}}^{\alpha^{\prime}} g_{i 1}\left(\xi^{\prime}, \lambda\right)\right| \leq C_{\alpha^{\prime}}\left(|\lambda|^{1 / 2}+\left|\xi^{\prime}\right|\right)^{-2-\left|\alpha^{\prime}\right|} \quad(i=1,2,3)
$$

for any $\alpha^{\prime} \in \mathbb{N}_{0}^{N-1}$ and $\left(\xi^{\prime}, \lambda\right) \in \Xi_{\vartheta, \sigma_{0}}^{\prime}$.

Moreover, by (4-4)

$$
\begin{aligned}
g_{11}\left(\xi^{\prime}, \lambda\right) & =\frac{1}{\lambda} \frac{1}{c_{1}+O(t)}\left[\left(1+\frac{t}{2 \gamma_{2}}\right)\left(1+\frac{t}{2 \gamma_{2}}\right)\left(\gamma_{2}^{2}-\gamma_{3}^{2}\right)\left(\gamma_{1} t-(1-\beta)\right)+O\left(t^{2}\right)\right] \\
& =\frac{1}{\lambda}\left(\mu_{11}+O(t)\right) \quad\left(t=\frac{\lambda}{\left|\xi^{\prime}\right|^{2}}\right)
\end{aligned}
$$


with $\mu_{11}=-\frac{\left(\gamma_{2}^{2}-\gamma_{3}^{2}\right)(1-\beta)}{c_{1}}$ for any $\left(\xi^{\prime}, \lambda\right) \in \Xi_{\vartheta, \sigma_{0}}$. By permutation of indices, we get the same result for $g_{21}, g_{31}$ with

$$
\mu_{21}=-\frac{\left(\gamma_{3}^{2}-\gamma_{1}^{2}\right)(1-\beta)}{c_{1}}, \quad \mu_{31}=-\frac{\left(\gamma_{1}^{2}-\gamma_{2}^{2}\right)(1-\beta)}{c_{1}},
$$

so that we have

$$
\left|\partial_{\xi^{\prime}}^{\alpha^{\prime}}\left(\lambda g_{i 1}\left(\xi^{\prime}, \lambda\right)-\mu_{i 1}\right)\right| \leq C_{\alpha^{\prime}}|\lambda|\left|\xi^{\prime}\right|^{-2-\left|\alpha^{\prime}\right|} \quad(i=1,2,3)
$$

for any $\alpha^{\prime} \in \mathbb{N}_{0}^{N-1}$ and $\left(\xi^{\prime}, \lambda\right) \in \Xi_{\vartheta, \sigma_{0}}$, which, combined with (4-7), furnishes that

$$
\left|\partial_{\xi^{\prime}}^{\alpha^{\prime}} \lambda g_{i 1}\left(\xi^{\prime}, \lambda\right)\right| \leq C_{\alpha^{\prime}}\left(|\lambda|^{-1 / 2}+\left|\xi^{\prime}\right|\right)^{-\left|\alpha^{\prime}\right|} \quad(i=1,2,3)
$$

for any $\alpha^{\prime} \in \mathbb{N}_{0}^{N-1}$ and any $\left(\xi^{\prime}, \lambda\right) \in \mathbb{R}^{N-1} \times \Sigma_{\vartheta}$. Here, we have used the fact: $\left|\xi^{\prime}\right|^{-1} \leq C\left(|\lambda|^{1 / 2}+\left|\xi^{\prime}\right|\right)^{-1}$ for $\left(\xi^{\prime}, \lambda\right) \in \mathbb{R}^{N-1} \times \Sigma_{\vartheta}$ with $|\lambda| \leq c_{0}\left|\xi^{\prime}\right|^{2}$.

Moreover, since $\mu_{11}+\mu_{21}+\mu_{31}=0$, by (4-8) we have

$$
\left|\partial_{\xi^{\prime}}^{\alpha^{\prime}}\left(\sum_{i=1}^{3} g_{i 1}\left(\xi^{\prime}, \lambda\right)\right)\right| \leq C_{\alpha^{\prime}}\left|\xi^{\prime}\right|^{-2-\left|\alpha^{\prime}\right|}
$$

for any $\alpha^{\prime} \in \mathbb{N}_{0}^{N-1}$ and $\left(\xi^{\prime}, \lambda\right) \in \Xi_{\vartheta, \sigma_{0}}$

(iv) Secondly, we estimate $g_{i 2}$. The coefficient of $g_{12}$ is given by

$$
g_{12}\left(\xi^{\prime}, \lambda\right)=\frac{\lambda}{\operatorname{det} \Delta\left(\xi^{\prime}, \lambda\right)}\left[\left(-\gamma_{3} \lambda+\zeta\right) A_{2}\left(\gamma_{2}^{2}+2\right)-\left(-\gamma_{2} \lambda+\zeta\right) A_{3}\left(\gamma_{3}^{2}+2\right)\right] .
$$

By (4-6), Leibniz rule, (3-15), (3-16) with $s=1$, and the permutation of indices, we have

$$
\left|\partial_{\xi^{\prime}}^{\alpha^{\prime}} g_{i 2}\left(\xi^{\prime}, \lambda\right)\right| \leq C_{\alpha^{\prime}}\left(|\lambda|^{1 / 2}+\left|\xi^{\prime}\right|\right)^{-3-\left|\alpha^{\prime}\right|} \quad(i=1,2,3)
$$

for any $\alpha^{\prime} \in \mathbb{N}_{0}^{N-1}$ and $\left(\xi^{\prime}, \lambda\right) \in \Xi_{\vartheta, \sigma_{0}}^{\prime}$

The asymptotic expansion for $t=\frac{\lambda}{\left|\xi^{\prime}\right|^{2}} \rightarrow 0$ is given by

$$
g_{12}\left(\xi^{\prime}, \lambda\right)=\frac{1}{\lambda\left|\xi^{\prime}\right|}\left(\frac{(1-\beta)\left(\gamma_{2}^{2}-\gamma_{3}^{2}\right)}{c_{1}}+O(t)\right)
$$

for any $\left(\xi^{\prime}, \lambda\right) \in \Xi_{\vartheta, \sigma_{0}}$. By permutation of indices, we get

$$
\mu_{12}=\frac{1}{c_{1}}(1-\beta)\left(\gamma_{2}^{2}-\gamma_{3}^{2}\right), \quad \mu_{22}=\frac{1}{c_{1}}(1-\beta)\left(\gamma_{3}^{2}-\gamma_{1}^{2}\right), \quad \mu_{32}=\frac{1}{c_{1}}(1-\beta)\left(\gamma_{1}^{2}-\gamma_{2}^{2}\right) .
$$

Thus, noting that $\mu_{12}+\mu_{22}+\mu_{23}=0$, we have

$$
\left|\partial_{\xi^{\prime}}^{\alpha^{\prime}} \lambda g_{i 2}\left(\xi^{\prime}, \lambda\right)\right| \leq C_{\alpha^{\prime}}\left|\xi^{\prime}\right|^{-1-\left|\alpha^{\prime}\right|}, \quad\left|\partial_{\xi^{\prime}}^{\alpha^{\prime}}\left(\sum_{i=1}^{3} g_{i 2}\left(\xi^{\prime}, \lambda\right)\right)\right| \leq C_{\alpha^{\prime}}\left|\xi^{\prime}\right|^{-3-\left|\alpha^{\prime}\right|}
$$

for any $\alpha^{\prime} \in \mathbb{N}_{0}^{N-1}$ and $\left(\xi^{\prime}, \lambda\right) \in \Xi_{\vartheta, \sigma_{0}}$. Moreover, using (4-10), we have

$$
\left|\partial_{\xi^{\prime}}^{\alpha^{\prime}} \lambda g_{i 2}\left(\xi^{\prime}, \lambda\right)\right| \leq C_{\alpha^{\prime}}\left(|\lambda|^{-1 / 2}+\left|\xi^{\prime}\right|\right)^{-1-\left|\alpha^{\prime}\right|} \quad(i=1,2,3)
$$

for any $\alpha^{\prime} \in \mathbb{N}_{0}^{N-1}$ and any $\left(\xi^{\prime}, \lambda\right) \in \mathbb{R}^{N-1} \times \Sigma_{\vartheta}$.

(v) Thirdly, we estimate $g_{i 3}$. The coefficient of $g_{13}$ is given by

$$
g_{13}\left(\xi^{\prime}, \lambda\right)=\frac{1}{\operatorname{det} \Delta\left(\xi^{\prime}, \lambda\right)}\left[\left(-\gamma_{2} \lambda+\zeta\right) A_{3}\left(-\frac{\lambda}{\gamma_{3}}+\zeta\right)-\left(-\gamma_{3} \lambda+\zeta\right) A_{2}\left(-\frac{\lambda}{\gamma_{2}}+\zeta\right)\right] .
$$

By (4-6), Leibniz rule, (3-15), (3-16) with $s=1$, and the permutation of indices, we have

$$
\left|\partial_{\xi^{\prime}}^{\alpha^{\prime}} g_{i 3}\left(\xi^{\prime}, \lambda\right)\right| \leq C_{\alpha^{\prime}}\left(|\lambda|^{1 / 2}+\left|\xi^{\prime}\right|\right)^{-3-\left|\alpha^{\prime}\right|} \quad(i=1,2,3)
$$


for any $\alpha^{\prime} \in \mathbb{N}_{0}^{N-1}$ and $\left(\xi^{\prime}, \lambda\right) \in \Xi_{\vartheta, \sigma_{0}}^{\prime}$.

The asymptotic expansion for $t=\frac{\lambda}{\left|\xi^{\prime}\right|^{2}} \rightarrow 0$ is given by

$$
\begin{aligned}
g_{13}\left(\xi^{\prime}, \lambda\right) & =\frac{\left|\xi^{\prime}\right|^{5}}{\lambda^{2}\left|\xi^{\prime}\right|^{4}\left(c_{1}+O(t)\right)}\left[\left((1-\beta)-\gamma_{2} t\right)\left(1+\frac{t}{2 \gamma_{3}}\right)\left((1-\beta)-\frac{t}{\gamma_{3}}\right)\right. \\
& \left.-\left((1-\beta)-\gamma_{3} t\right)\left(1+\frac{t}{2 \gamma_{2}}\right)\left((1-\beta)-\frac{t}{\gamma_{2}}\right)+O\left(t^{2}\right)\right] \\
& =\frac{\left|\xi^{\prime}\right|}{\lambda^{2}\left(c_{1}+O(t)\right)}\left[d_{1} t+O\left(t^{2}\right)\right]=\frac{1}{\lambda\left|\xi^{\prime}\right|}\left[\frac{d_{1}}{c_{1}}+O(t)\right]
\end{aligned}
$$

for any $\left(\xi^{\prime}, \lambda\right) \in \Xi_{\vartheta, \sigma_{0}}$, where

$$
\begin{aligned}
d_{1} & =\left(-\gamma_{2}(1-\beta)+\frac{1}{2 \gamma_{3}}(1-\beta)^{2}-\frac{1}{\gamma_{3}}(1-\beta)\right) \\
& -\left(-\gamma_{3}(1-\beta)+\frac{1}{2 \gamma_{2}}(1-\beta)^{2}-\frac{1}{\gamma_{2}}(1-\beta)\right) \\
& =(1-\beta)\left(\gamma_{3}-\gamma_{2}\right)-(1-\beta)\left(\frac{1}{2}+\frac{\beta}{2}\right)\left(\frac{1}{\gamma_{3}}-\frac{1}{\gamma_{2}}\right) .
\end{aligned}
$$

It is easily seen that $d_{1} \neq 0$, and we get $g_{13}\left(\xi^{\prime}, \lambda\right)=\frac{1}{\lambda\left|\xi^{\prime}\right|}\left(\mu_{31}+O(t)\right)$ with

$$
\mu_{31}=\frac{1}{c_{1}}(1-\beta)\left(\gamma_{3}-\gamma_{2}-\left(\frac{1}{2}+\frac{\beta}{2}\right)\left(\frac{1}{\gamma_{3}}-\frac{1}{\gamma_{2}}\right)\right)
$$

for $\left(\xi^{\prime}, \lambda\right) \in \Xi_{\vartheta, \sigma_{0}}$. For $g_{23}$ and $g_{33}$ we obtain the same result with

$$
\begin{aligned}
& \mu_{32}=\frac{1}{c_{1}}(1-\beta)\left(\gamma_{1}-\gamma_{3}-\left(\frac{1}{2}+\frac{\beta}{2}\right)\left(\frac{1}{\gamma_{1}}-\frac{1}{\gamma_{3}}\right)\right), \\
& \mu_{33}=\frac{1}{c_{1}}(1-\beta)\left(\gamma_{2}-\gamma_{1}-\left(\frac{1}{2}+\frac{\beta}{2}\right)\left(\frac{1}{\gamma_{2}}-\frac{1}{\gamma_{1}}\right)\right),
\end{aligned}
$$

Thus, noting that $\mu_{31}+\mu_{32}+\mu_{33}=0$, we have

$$
\left|\partial_{\xi^{\prime}}^{\alpha^{\prime}} \lambda g_{i 3}\left(\xi^{\prime}, \lambda\right)\right| \leq C_{\alpha^{\prime}}\left|\xi^{\prime}\right|^{-1-\left|\alpha^{\prime}\right|}, \quad\left|\partial_{\xi^{\prime}}^{\alpha^{\prime}}\left(\sum_{i=1}^{3} g_{i 3}\left(\xi^{\prime}, \lambda\right)\right)\right| \leq C_{\alpha^{\prime}}\left|\xi^{\prime}\right|^{-3-\left|\alpha^{\prime}\right|}
$$

for any $\alpha^{\prime} \in \mathbb{N}_{0}^{N-1}$ and $\left(\xi^{\prime}, \lambda\right) \in \Xi_{\vartheta, \sigma_{0}}$. Moreover, using (4-11), we have

$$
\left|\partial_{\xi^{\prime}}^{\alpha^{\prime}} \lambda g_{i 3}\left(\xi^{\prime}, \lambda\right)\right| \leq C_{\alpha^{\prime}}\left(|\lambda|^{-1 / 2}+\left|\xi^{\prime}\right|\right)^{-1-\left|\alpha^{\prime}\right|} \quad(i=1,2,3)
$$

for any $\alpha^{\prime} \in \mathbb{N}_{0}^{N-1}$ and any $\left(\xi^{\prime}, \lambda\right) \in \mathbb{R}^{N-1} \times \Sigma_{\vartheta}$.

Analogously, we can treat $\tau \partial_{\tau} g_{i j}\left(\xi^{\prime}, \lambda\right)$, so that we have proved Theorem 3.4.

\section{Problem in a bent half-space}

Let $\Phi$ be a diffeomorphism of class $H_{\infty}^{4}$ from $\mathbb{R}^{N}$ onto itself and let $\Phi^{-1}$ be its inverse operator. Let $\nabla \Phi(x)=\mathcal{A}+B(x)$ and $\nabla \Phi^{-1}(y)=\mathcal{A}_{-1}+B_{-1}(y)$, where we assume that $\mathcal{A}$ and $\mathcal{A}_{-1}$ are orthonormal matrices with constant coefficients and $B(x)$ and $B_{-1}(y)$ are matrices of $H_{\infty}^{3}\left(\mathbb{R}^{N}\right)$-functions which satisfy the conditions:

$$
\left\|\left(B, B_{-1}\right)\right\|_{L_{\infty}\left(\mathbb{R}^{N}\right)} \leq M_{1}, \quad\left\|\nabla\left(B, B_{-1}\right)\right\|_{H_{\infty}^{2}\left(\mathbb{R}^{N}\right)} \leq M_{2} .
$$

We choose $M_{1}$ small enough eventually, so that we may assume that $0<M_{1} \leq 1 \leq M_{2}$ without loss of generality. Let $\Omega_{+}=\Phi\left(\mathbb{R}_{+}^{N}\right)$ and $\Gamma_{+}=\Phi\left(\mathbb{R}_{0}^{N}\right)$ and let $\nu_{+}$be the unit outer normal to $\Gamma_{+}$.

In this section, we consider the equations:

$$
\lambda U-A(D) U=F \quad \text { in } \Omega_{+}, \quad B(D) U=G \quad \text { on } \Gamma_{+}
$$

with $F=\left(0, f_{1}, f_{2}\right)^{\top}$ and $G=\left(g_{1}, g_{2}, g_{3}\right)^{\top}$. The purpose of this section is to prove 
Theorem 5.1. Let $1<q<\infty$ and let $\vartheta$ be the same number as in Theorem 3.1. Let $M_{1}$ in $(5-1)$ be sufficiently small. Then, there exist a constant $\lambda_{1}>0$ and operator families $\mathcal{T}_{+i}(\lambda)(i=1,2)$ with

$$
\mathcal{T}_{+1}(\lambda) \in \mathcal{C}\left(\Sigma_{\vartheta, \lambda_{1}}, \mathcal{L}\left(\mathcal{X}_{q}\left(\Omega_{+}\right), H_{q}^{4}\left(\Omega_{+}\right)\right)\right), \quad \mathcal{T}_{+2}(\lambda) \in \mathcal{C}\left(\Sigma_{\vartheta, \lambda_{1}}, \mathcal{L}\left(\mathcal{X}_{q}\left(\Omega_{+}\right), H_{q}^{2}\left(\Omega_{+}\right)\right)\right)
$$

such that problem (5-2) admits a unique solution

$$
U=\left(\mathcal{T}_{+1}(\lambda) H_{\lambda}(F, G), \lambda \mathcal{T}_{+1}(\lambda) H_{\lambda}(F, G), \mathcal{T}_{+2}(\lambda) H_{\lambda}(F, G)\right)^{\top}
$$

for any $(F, G) \in \mathbb{G}_{q}\left(\Omega_{+}\right)$and $\lambda \in \Sigma_{\vartheta, \lambda_{1}}$, and there hold the estimates:

$$
\begin{aligned}
& \mathcal{R}_{\mathcal{L}\left(\mathcal{X}_{q}\left(\Omega_{+}\right), H_{q}^{4-j}\left(\Omega_{+}\right)\right)}\left(\left\{\left(\tau \partial_{\tau}\right)^{s}\left(\lambda^{j / 2} \mathcal{T}_{+1}(\lambda)\right) \mid \lambda \in \Sigma_{\vartheta, \lambda_{0}}\right\}\right) \leq C_{N, q, \lambda_{0}} \quad(s=0,1, \quad j=0,1,2,3,4), \\
& \mathcal{R}_{\mathcal{L}\left(\mathcal{X}_{q}\left(\Omega_{+}\right), H_{q}^{2-j}\left(\Omega_{+}\right)\right)}\left(\left\{\left(\tau \partial_{\tau}\right)^{s}\left(\lambda^{j / 2} \mathcal{T}_{+2}(\lambda)\right) \mid \lambda \in \Sigma_{\vartheta, \lambda_{0}}\right\}\right) \leq C_{N, q, \lambda_{0}} \quad(s=0,1, \quad j=0,1,2) .
\end{aligned}
$$

In what follows, we prove Theorem 5.1. We use the change of variables: $y=\Phi(x)$ to transfer problem (5-2) to the half space case. Let

$$
\frac{\partial x_{j}}{\partial y_{k}}(\Phi(x))=A_{j k}+B_{j k}(x)
$$

and then, by (5-1)

$$
\sum_{j=1}^{N} A_{j k} A_{j \ell}=\sum_{j=1}^{N} A_{k j} A_{\ell j}=\delta_{k \ell}, \quad\left\|B_{j k}\right\|_{L_{\infty}\left(\mathbb{R}^{N}\right)} \leq M_{1}, \quad\left\|\nabla B_{j k}\right\|_{H_{\infty}^{2}\left(\mathbb{R}^{N}\right)} \leq M_{2} .
$$

Since $\Gamma_{+}$is represented by $x_{N}=\Phi_{-, N}(y)=0$ with $\Phi^{-1}=\left(\Phi_{-, 1}, \ldots, \Phi_{-, N}\right)$, we have

$$
\nu_{+}(y)=-d^{-1}\left(\frac{\partial x_{N}}{\partial y_{1}}, \ldots, \frac{\partial x_{N}}{\partial y_{N}}\right)(y) \quad\left(y \in \Gamma_{+}\right)
$$

with $d=\sqrt{\sum_{j=1}^{N}\left(\partial x_{N} / \partial y_{j}\right)^{2}}$. By (5-5), we may assume that $\nu_{+}$is defined in $\mathbb{R}^{N}$. Moreover, choosing $M_{1}>0$ sufficiently small, by (5-3) and (5-4) we have

$$
\nu_{+}=-\left(A_{N 1}, \ldots, A_{N N}\right)+\tilde{\nu}_{+}
$$

with some vector of functions $\tilde{\nu}_{+}$defined on $\mathbb{R}^{N}$ satisying the estimate:

$$
\left\|\tilde{\nu}_{+}\right\|_{L_{\infty}\left(\mathbb{R}^{N}\right)} \leq C M_{1}, \quad\left\|\nabla \tilde{\nu}_{+}\right\|_{H_{\infty}^{2}\left(\mathbb{R}^{N}\right)} \leq C_{M_{2}}
$$

where $C_{M_{2}}$ is some constant depending on $M_{2}$. Using the relation

$$
\frac{\partial}{\partial y_{j}}=\sum_{k=1}^{N} \frac{\partial x_{k}}{\partial y_{j}} \frac{\partial}{\partial x_{k}}=\sum_{k=1}^{N}\left(A_{k j}+B_{k j}(x)\right) \frac{\partial}{\partial x_{k}}
$$

by (5-4) we have

$$
\Delta_{y}=\Delta_{x}+E^{1}(D), \quad \Delta_{y}^{2}=\Delta_{x}^{2}+E^{2}(D),
$$

where $E^{1}(D)$ and $E^{2}(D)$ are some partial differential operators of the form:

$$
E^{1}(D)=\sum_{1 \leq|\alpha| \leq 2} e_{\alpha}^{1}(x) \partial_{x}^{\alpha}, \quad E^{2}(D)=\sum_{1 \leq|\alpha| \leq 4} e_{\alpha}^{2}(x) \partial_{x}^{\alpha},
$$

with

$$
\begin{aligned}
& \left\|e_{\alpha}^{1}\right\|_{L_{\infty}\left(\mathbb{R}^{N}\right)} \leq C M_{1}(|\alpha|=2), \quad\left\|e_{\alpha}^{2}\right\|_{L_{\infty}\left(\mathbb{R}^{N}\right)} \leq C M_{1} \quad(|\alpha|=4), \\
& \left\|\nabla e_{\alpha}^{1}\right\|_{H_{\infty}^{2}\left(\mathbb{R}^{N}\right)} \leq C_{M_{2}}(|\alpha|=2), \quad\left\|\nabla e_{\alpha}^{2}\right\|_{H_{\infty}^{2}\left(\mathbb{R}^{N}\right)} \leq C_{M_{2}}(|\alpha|=4), \\
& \left\|e_{\alpha}^{1}\right\|_{H_{\infty}^{2}\left(\mathbb{R}^{N}\right)} \leq C_{M_{2}}(|\alpha|=1), \quad\left\|e_{\alpha}^{2}\right\|_{H_{\infty}^{|\alpha|-1}\left(\mathbb{R}^{N}\right)} \leq C_{M_{2}}(1 \leq|\alpha| \leq 3) \text {. }
\end{aligned}
$$


Moreover, by (5-1), (5-4), (5-6) and (5-7),

$$
\partial_{\nu_{+}} \circ \Phi=-\partial_{N}+E^{3}(D), \quad \Delta^{\prime}=\Delta_{x^{\prime}}+E^{4}(D)
$$

where $\Delta_{x^{\prime}}=\sum_{j=1}^{N-1} \partial_{j}^{2}$, and $E^{3}(D)$ and $E^{4}(D)$ are some partial differential operators of the form:

$$
E^{3}(D)=\sum_{|\alpha|=1} e_{\alpha}^{3} \partial_{x}^{\alpha}, \quad E^{4}(D)=\sum_{j, k=1}^{N-1} e_{j k}^{4} \partial_{j} \partial_{k}+\sum_{j=1}^{N-1} e_{j}^{4} \partial_{j} .
$$

with

$$
\begin{gathered}
\left\|e_{\alpha}^{3}\right\|_{L_{\infty}\left(\mathbb{R}^{N}\right)} \leq C M_{1}, \quad\left\|\nabla e_{\alpha}^{3}\right\|_{H_{\infty}^{2}\left(\mathbb{R}^{N}\right)} \leq C_{M_{2}}, \\
\left\|e_{j k}^{4}\right\|_{L_{\infty}\left(\mathbb{R}^{N}\right)} \leq C M_{1}, \quad\left\|\left(\nabla e_{j k}^{4}, e_{j}^{4}\right)\right\|_{H_{\infty}^{2}\left(\mathbb{R}^{N}\right)} \leq C_{M_{2}} .
\end{gathered}
$$

Thus, problem (5-2) is transformed to

$$
\lambda U-A(D) U-P(D) U=\tilde{F} \quad \text { in } \mathbb{R}_{+}^{N}, \quad B^{\prime}(D) U-Q(D) U=\tilde{G} \quad \text { on } \mathbb{R}_{0}^{N}
$$

with $\tilde{F}=F \circ \Phi, \tilde{G}=\left(g_{1} \circ \Phi,-g_{2} \circ \Phi,-g_{3} \circ \Phi\right)^{\top}$. For simplicity, we continue to write $F$ and $G$ instead of $\tilde{F}$ and $\tilde{G}$, respectively.

$$
\begin{aligned}
& P(D)=\left(\begin{array}{ccc}
0 & 0 & 0 \\
-E^{2}(D) & 0 & -E^{1}(D) \\
0 & E^{1}(D) & E^{1}(D)
\end{array}\right) \quad B^{\prime}(D)=\left(\begin{array}{ccc}
\Delta-(1-\beta) \Delta_{x^{\prime}}^{\prime} & 0 & 1 \\
\partial_{N}\left(\Delta+(1-\beta) \Delta_{x^{\prime}}\right) & 0 & 0 \\
0 & 0 & \partial_{N}
\end{array}\right), \\
& Q(D)=-\left(\begin{array}{ccc}
E^{1}(D)-(1-\beta) E^{4}(D) & 0 & 0 \\
Q_{21}(D) & 0 & 0 \\
0 & 0 & E^{3}(D)
\end{array}\right) \text {, } \\
& Q_{21}(D)=E^{3}(D)\left(\Delta+(1-\beta) \Delta_{x^{\prime}}\right)+\left(\partial_{N}+E^{3}(D)\right)\left(E^{1}(D)+(1-\beta) E^{4}(D)\right) .
\end{aligned}
$$

Recall that

$$
H_{\lambda}(F, G)=\left(f_{1}, f_{2}, G, \lambda^{1 / 2} G, \lambda g_{1}\right) \quad \text { for }(F, G) \in \mathbb{G}_{q}\left(\mathbb{R}_{+}^{N}\right) \text { with } F=\left(0, f_{1}, f_{2}\right)^{\top} \text { and } G=\left(g_{1}, g_{2}, g_{3}\right)^{\top} \text {. }
$$

Let $\mathcal{T}_{i}(\lambda)(i=1,2)$ be the operators given in Theorem 3.1. Setting $\mathcal{T}(\lambda):=\left(\mathcal{T}_{1}(\lambda), \lambda \mathcal{T}_{1}(\lambda), \mathcal{T}_{2}(\lambda)\right)^{\top}$ and $\tilde{U}:=\mathcal{T}(\lambda) H_{\lambda}(F, G)$, we have

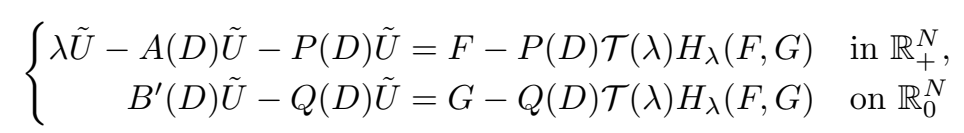

with $F=\left(0, f_{1}, f_{2}\right)^{\top}$. Let

$$
\begin{aligned}
& L_{\lambda}(D)=\left(\lambda \mathbf{I}-A(D)-P(D), \quad B^{\prime}(D)-Q(D)\right), \\
& \mathcal{U}(\lambda)\left(F^{\prime}, G, G^{\prime}, g_{1}^{\prime \prime}\right)=\left(P(D) \mathcal{T}(\lambda)\left(F^{\prime}, G, G^{\prime}, g_{1}^{\prime \prime}\right), Q(D) \mathcal{T}(\lambda)\left(F^{\prime}, G, G^{\prime}, g_{1}^{\prime \prime}\right)\right),
\end{aligned}
$$

where $\mathbf{I}$ is the $3 \times 3$ identity matrix. Then, problem (5-14) can be written in the form

$$
L_{\lambda}(D) \mathcal{T}(\lambda) H_{\lambda}(F, G)=(F, G)-\mathcal{U}(\lambda) H_{\lambda}(F, G)=\left(I-\mathcal{U}(\lambda) H_{\lambda}\right)(F, G) \quad \text { on } \mathbb{R}_{+}^{N} \times \mathbb{R}_{0}^{N}
$$

with $F=\left(0, f_{1}, f_{2}\right)^{\top}$ and $G=\left(g_{1}, g_{2}, g_{3}\right)^{\top}$. In the following, we will show that $I-H_{\lambda} \mathcal{U}(\lambda)$ is invertible.

Noting that

$$
P(D) \mathcal{T}(\lambda)\left(F^{\prime}, G, G^{\prime}, g_{1}^{\prime \prime}\right)=\left(\begin{array}{c}
0 \\
-E^{2}(D) \mathcal{T}_{1}(\lambda)\left(F^{\prime}, G, G^{\prime}, g_{1}^{\prime \prime}\right)-E^{1}(D) \mathcal{T}_{2}(\lambda)\left(F^{\prime}, G, G^{\prime}, g_{1}^{\prime \prime}\right) \\
E^{1}(D) \lambda \mathcal{T}_{1}(\lambda)\left(F^{\prime}, G, G^{\prime}, g_{1}^{\prime \prime}\right)+E^{1}(D) \mathcal{T}_{2}(\lambda)\left(F^{\prime}, G, G^{\prime}, g_{1}^{\prime \prime}\right)
\end{array}\right)
$$


we have

$$
H_{\lambda} \mathcal{U}(\lambda)\left(F^{\prime}, G, G^{\prime}, g_{1}^{\prime \prime}\right)=\left(\begin{array}{c}
-E^{2}(D) \mathcal{T}_{1}(\lambda)\left(F^{\prime}, G, G^{\prime}, g_{1}^{\prime \prime}\right)-E^{1}(D) \mathcal{T}_{2}(\lambda)\left(F^{\prime}, G, G^{\prime}, g_{1}^{\prime \prime}\right) \\
E^{1}(D) \lambda \mathcal{T}_{1}(\lambda)\left(F^{\prime}, G, G^{\prime}, g_{1}^{\prime \prime}\right)+E^{1}(D) \mathcal{T}_{2}(\lambda)\left(F^{\prime}, G, G^{\prime}, g_{1}^{\prime \prime}\right) \\
Q(D) \mathcal{T}(\lambda)\left(F^{\prime}, G, G^{\prime}, g_{1}^{\prime \prime}\right) \\
\lambda^{1 / 2} Q(D) \mathcal{T}(\lambda)\left(F^{\prime}, G, G^{\prime}, g_{1}^{\prime \prime}\right) \\
\lambda\left(E^{1}(D) \mathcal{T}_{1}(\lambda)-(1-\beta) E^{4}(D)\right) \mathcal{T}_{1}(\lambda)\left(F^{\prime}, G, G^{\prime}, g_{1}^{\prime \prime}\right)
\end{array}\right)
$$

Recall the definition of $\mathcal{X}_{q}(D)$ given in (1-7). By Theorem 3.1, Proposition 2.2, (5-10) and (5-12), we will show that

$$
\mathcal{R}_{\mathcal{L}\left(\mathcal{X}_{q}\left(\mathbb{R}_{+}^{N}\right)\right)}\left(\left\{\left(\tau \partial_{\tau}\right)^{s}\left(H_{\lambda} \mathcal{U}(\lambda)\right) \mid \lambda \in \Sigma_{\vartheta, \lambda_{1}}\right\}\right) \leq C M_{1}+C_{M_{2}} \lambda_{1}^{-1 / 2} \quad(s=0,1)
$$

holds for any $\lambda_{1} \geq \lambda_{0}$. Here, $\lambda_{0}$ is the same number as in Theorem 3.1, and $C$ and $C_{M_{2}}$ are constants, where $C$ is independent of $M_{1}, M_{2}$ and $\lambda_{1}$ and $C_{M_{2}}$ independent of $M_{1}$ and $\lambda_{1}$. In fact,

$$
\begin{aligned}
& \int_{0}^{1}\left\|\sum_{k=1}^{n} r_{k}(\omega) P(D) \mathcal{T}\left(\lambda_{k}\right)\left(F_{k}^{\prime}, G_{k}, G_{k}^{\prime}, g_{1 k}^{\prime \prime}\right)\right\|_{L_{q}\left(\mathbb{R}_{+}^{N}\right)^{3}}^{q} d \omega \\
& \quad \leq C \int_{0}^{1}\left\{M_{1}\left\|\nabla^{4}\left(\sum_{k=1}^{n} r_{k}(\omega) \mathcal{T}_{1}\left(\lambda_{k}\right)\left(F_{k}^{\prime}, G_{k}, G_{k}^{\prime}, g_{1 k}^{\prime \prime}\right)\right)\right\|_{L_{q}\left(\mathbb{R}_{+}^{N}\right)}^{q}\right. \\
& \quad+C_{M_{2}}\left\|\sum_{k=1}^{n} r_{k}(\omega) \mathcal{T}_{1}\left(\lambda_{k}\right)\left(F_{k}^{\prime}, G_{k}, G_{k}^{\prime}, g_{1 k}^{\prime \prime}\right)\right\|_{H_{q}^{3}\left(\mathbb{R}_{+}^{N}\right)}^{q} \\
& \quad+M_{1}\left\|\nabla^{2}\left(\sum_{k=1}^{n} r_{k}(\omega) \lambda_{k} \mathcal{T}_{1}\left(\lambda_{k}\right)\left(F_{k}^{\prime}, G_{k}, G_{k}^{\prime}, g_{1 k}^{\prime \prime}\right)\right)\right\|_{L_{q}\left(\mathbb{R}_{+}^{N}\right)}^{q} \\
& \quad+C_{M_{2}}\left\|\sum_{k=1}^{n} r_{k}(\omega) \lambda_{k} \mathcal{T}_{1}\left(\lambda_{k}\right)\left(F_{k}^{\prime}, G_{k}, G_{k}^{\prime}, g_{1 k}^{\prime \prime}\right)\right\|_{H_{q}^{1}\left(\mathbb{R}_{+}^{N}\right)}^{q} \\
& \quad+M_{1}\left\|\nabla^{2}\left(\sum_{k=1}^{n} r_{k}(\omega) \mathcal{T}_{2}\left(\lambda_{k}\right)\left(F_{k}^{\prime}, G_{k}, G_{k}^{\prime}, g_{1 k}^{\prime \prime}\right)\right)\right\|_{L_{q}\left(\mathbb{R}_{+}^{N}\right)}^{q} \\
& \left.\quad+C_{M_{2}}\left\|\sum_{k=1}^{n} r_{k}(\omega) \mathcal{T}_{2}\left(\lambda_{k}\right)\left(F_{k}^{\prime}, G_{k}, G_{k}^{\prime}, g_{1 k}^{\prime \prime}\right)\right\|_{H_{q}^{1}\left(\mathbb{R}_{+}^{N}\right)}^{q}\right\} d \omega \\
& \leq\left(C M_{1}+C_{M_{2}} \lambda_{1}^{-1 / 2}\right) \int_{0}^{1}\left\|\sum_{k=1}^{n} r_{k}(\omega)\left(F_{k}^{\prime}, G_{k}, G_{k}^{\prime}, g_{1 k}^{\prime \prime}\right)\right\|_{\mathcal{X}_{q}\left(\mathbb{R}_{+}^{N}\right)}^{q} d \omega
\end{aligned}
$$

which, combined with Theorem 3.1, furnishes that

$$
\mathcal{R}_{\mathcal{L}\left(\mathcal{X}_{q}\left(\mathbb{R}_{+}^{N}\right), L q\left(\mathbb{R}_{+}^{N}\right)^{3}\right)}\left(\left\{P(D) \mathcal{T}(\lambda) \mid \lambda \in \Sigma_{\vartheta, \lambda_{1}}\right\}\right) \leq C M_{1}+C_{M_{2}} \lambda_{1}^{-1 / 2}
$$

for any $\lambda_{1} \geq \lambda_{0}$. By Theorem 3.1, Proposition 2.2, (5-10), and (5-12),

$$
\begin{aligned}
& \int_{0}^{1}\left\|\sum_{k=1}^{n} r_{k}(\omega) Q(D) \mathcal{T}\left(\lambda_{k}\right)\left(F_{k}^{\prime}, G_{k}, G_{k}^{\prime}, g_{1 k}^{\prime \prime}\right)\right\|_{H_{q}^{2}\left(\mathbb{R}_{+}^{N}\right) \times H_{q}^{1}\left(\mathbb{R}_{+}^{N}\right)^{2}}^{q} d \omega \\
& \quad \leq C \int_{0}^{1}\left\{M_{1}\left\|\nabla^{4}\left(\sum_{k=1}^{n} r_{k}(\omega) \mathcal{T}_{1}\left(\lambda_{k}\right)\left(F_{k}^{\prime}, G_{k}, G_{k}^{\prime}, g_{1 k}^{\prime \prime}\right)\right)\right\|_{L_{q}\left(\mathbb{R}_{+}^{N}\right)}^{q}\right. \\
& \quad+C_{M_{2}}\left\|\sum_{k=1}^{n} r_{k}(\omega) \mathcal{T}_{1}\left(\lambda_{k}\right)\left(F_{k}^{\prime}, G_{k}, G_{k}^{\prime}, g_{1 k}^{\prime \prime}\right)\right\|_{H_{q}^{3}\left(\mathbb{R}_{+}^{N}\right)}^{q} \\
& \quad+M_{1}\left\|\nabla^{2}\left(\sum_{k=1}^{n} r_{k}(\omega) \mathcal{T}_{2}\left(\lambda_{k}\right)\left(F_{k}^{\prime}, G_{k}, G_{k}^{\prime}, g_{1 k}^{\prime \prime}\right)\right)\right\|_{L_{q}\left(\mathbb{R}_{+}^{N}\right)}^{q}
\end{aligned}
$$




$$
\begin{aligned}
&\left.+C_{M_{2}}\left\|\sum_{k=1}^{n} r_{k}(\omega) \mathcal{T}_{2}\left(\lambda_{k}\right)\left(F_{k}^{\prime}, G_{k}, G_{k}^{\prime}, g_{1 k}^{\prime \prime}\right)\right\|_{H_{q}^{1}\left(\mathbb{R}_{+}^{N}\right)}^{q}\right\} d \omega \\
& \leq\left(C M_{1}+C_{M_{2}} \lambda_{1}^{-1 / 2}\right) \int_{0}^{1}\left\|\sum_{k=1}^{n} r_{k}(\omega)\left(F_{k}^{\prime}, G_{k}, G_{k}^{\prime}, g_{1 k}^{\prime \prime}\right)\right\|_{\mathcal{X}_{q}\left(\mathbb{R}_{+}^{N}\right)}^{q} d \omega ; \\
& \int_{0}^{1}\left\|\sum_{k=1}^{n} r_{k}(\omega) \lambda_{k}^{1 / 2} Q(D) \mathcal{T}\left(\lambda_{k}\right)\left(F_{k}^{\prime}, G_{k}, G_{k}^{\prime}, g_{1 k}^{\prime \prime}\right)\right\|_{H_{q}^{1}\left(\mathbb{R}_{+}^{N}\right) \times L_{q}\left(\mathbb{R}_{+}^{N}\right)^{2}}^{q} d \omega \\
& \quad \leq C \int_{0}^{1}\left\{M_{1}\left\|\nabla^{3}\left(\sum_{k=1}^{n} r_{k}(\omega) \lambda_{k}^{1 / 2} \mathcal{T}_{1}\left(\lambda_{k}\right)\left(F_{k}^{\prime}, G_{k}, G_{k}^{\prime}, g_{1 k}^{\prime \prime}\right)\right)\right\|_{L_{q}\left(\mathbb{R}_{+}^{N}\right)}^{q}\right. \\
& \quad+C_{M_{2}}\left\|\sum_{k=1}^{n} r_{k}(\omega) \lambda_{k}^{1 / 2} \mathcal{T}_{1}\left(\lambda_{k}\right)\left(F_{k}^{\prime}, G_{k}, G_{k}^{\prime}, g_{1 k}^{\prime \prime}\right)\right\|_{H_{q}^{2}\left(\mathbb{R}_{+}^{N}\right)}^{q} \\
& \quad+M_{1}\left\|\nabla\left(\sum_{k=1}^{n} r_{k}(\omega) \lambda_{k}^{1 / 2} \mathcal{T}_{2}\left(\lambda_{k}\right)\left(F_{k}^{\prime}, G_{k}, G_{k}^{\prime}, g_{1 k}^{\prime \prime}\right)\right)\right\|_{L_{q}\left(\mathbb{R}_{+}^{N}\right)}^{q} \\
&\left.\quad+C_{M_{2}}\left\|\sum_{k=1}^{n} r_{k}(\omega) \lambda_{k}^{1 / 2} \mathcal{T}_{2}\left(\lambda_{k}\right)\left(F_{k}^{\prime}, G_{k}, G_{k}^{\prime}, g_{1 k}^{\prime \prime}\right)\right\|_{L_{q}\left(\mathbb{R}_{+}^{N}\right)}^{q}\right\} d \omega \\
& \leq\left(C M_{1}+C_{M_{2}} \lambda_{1}^{-1 / 2}\right)\left\|\sum_{k=1}^{n} r_{k}(\omega)\left(F_{k}^{\prime}, G_{k}, G_{k}^{\prime}, g_{1 k}^{\prime \prime}\right)\right\|_{\mathcal{X}_{q}\left(\mathbb{R}_{+}^{N}\right)}^{q} d \omega .
\end{aligned}
$$

Moreover, setting $A^{1}(\lambda)=\left(E^{1}(D)-(1-\beta) E^{4}(D)\right) \mathcal{T}_{1}(\lambda)$, which is the first component of $Q(D) \mathcal{T}(\lambda)$, we have

$$
\begin{aligned}
& \int_{0}^{1}\left\|\sum_{k=1}^{n} r_{k}(\omega) \lambda_{k} A^{1}\left(\lambda_{k}\right)\left(F_{k}^{\prime}, G_{k}, G_{k}^{\prime}, g_{1 k}^{\prime \prime}\right)\right\|_{L_{q}\left(\mathbb{R}_{+}^{N}\right)}^{q} d \omega \\
& \quad \leq C \int_{0}^{1}\left\{M_{1}\left\|\nabla^{2}\left(\sum_{k=1}^{n} r_{k}(\omega) \lambda_{k} \mathcal{T}_{1}\left(\lambda_{k}\right)\left(F_{k}^{\prime}, G_{k}, G_{k}^{\prime}, g_{1 k}^{\prime \prime}\right)\right)\right\|_{L_{q}\left(\mathbb{R}_{+}^{N}\right)}^{q}\right. \\
& \left.\quad+C_{M_{2}}\left\|\sum_{k=1}^{n} r_{k}(\omega) \lambda_{k} \mathcal{T}_{1}\left(\lambda_{k}\right)\left(F_{k}^{\prime}, G_{k}, G_{k}^{\prime}, g_{1 k}^{\prime \prime}\right)\right\|_{H_{q}^{1}\left(\mathbb{R}_{+}^{N}\right)}^{q}\right\} d \omega \\
& \leq\left(C M_{1}+C_{M_{2}}\right) \lambda_{1}^{-1 / 2} \int_{0}^{1}\left\|\sum_{k=1}^{n} r_{k}(\omega)\left(F_{k}^{\prime}, G_{k}, G_{k}^{\prime}, g_{1 k}^{\prime \prime}\right)\right\|_{\mathcal{X}_{q}\left(\mathbb{R}_{+}^{N}\right)}^{q} d \omega .
\end{aligned}
$$

Therefore, we have

$$
\begin{aligned}
\mathcal{R}_{\mathcal{L}\left(\mathcal{X}_{q}\left(\mathbb{R}_{+}^{N}\right), H_{q}^{2}\left(\mathbb{R}_{+}^{N}\right) \times H_{q}^{1}\left(\mathbb{R}_{+}^{N}\right)^{2}\right)}\left(\left\{Q(D) \mathcal{T}(\lambda) \mid \lambda \in \Sigma_{\vartheta, \lambda_{1}}\right\}\right) \leq C M_{1}+C_{M_{2}} \lambda_{1}^{-1 / 2}, \\
\mathcal{R}_{\mathcal{L}\left(\mathcal{X}_{q}\left(\mathbb{R}_{+}^{N}\right), H_{q}^{1}\left(\mathbb{R}_{+}^{N}\right) \times L_{q}\left(\mathbb{R}_{+}^{N}\right)^{2}\right)}\left(\left\{\lambda^{1 / 2} Q(D) \mathcal{T}(\lambda) \mid \lambda \in \Sigma_{\vartheta, \lambda_{1}}\right\}\right) \leq C M_{1}+C_{M_{2}} \lambda_{1}^{-1 / 2}, \\
\mathcal{R}_{\mathcal{L}\left(\mathcal{X}_{q}\left(\mathbb{R}_{+}^{N}\right), L_{q}\left(\mathbb{R}_{+}^{N}\right)\right)}\left(\left\{\lambda A_{1}(\lambda) \mid \lambda \in \Sigma_{\vartheta, \lambda_{1}}\right\}\right) \leq C M_{1}+C_{M_{2}} \lambda_{1}^{-1 / 2} .
\end{aligned}
$$

Summing up, we have proved that

$$
\mathcal{R}_{\mathcal{L}\left(\mathcal{X}_{q}\left(\mathbb{R}_{+}^{N}\right)\right)}\left(\left\{H_{\lambda} \mathcal{U}(\lambda) \mid \lambda \in \Sigma_{\vartheta, \lambda_{1}}\right\}\right) \leq C M_{1}+C_{M_{2}} \lambda_{1}^{-1 / 2} .
$$

Analogously, we have

$$
\mathcal{R}_{\mathcal{L}\left(\mathcal{X}_{q}\left(\mathbb{R}_{+}^{N}\right)\right)}\left(\left\{\tau \partial_{\tau}\left(H_{\lambda} \mathcal{U}(\lambda)\right) \mid \lambda \in \Sigma_{\vartheta, \lambda_{1}}\right\}\right) \leq C M_{1}+C_{M_{2}} \lambda_{1}^{-1 / 2} .
$$

Therefore, we have obtained (5-16). 
Choosing $M_{1}>0$ so small that $C M_{1}<1 / 4$ and $\lambda_{1} \geq \lambda_{0}$ so large that $C_{M_{2}} \lambda_{1}^{-1 / 2} \leq 1 / 4$, respectively, we have

$$
\mathcal{R}_{\mathcal{L}\left(\mathcal{X}_{q}\left(\mathbb{R}_{+}^{N}\right)\right)}\left(\left\{\left(\tau \partial_{\tau}\right)^{s}\left(H_{\lambda} \mathcal{U}(\lambda)\right) \mid \lambda \in \Sigma_{\vartheta, \lambda_{1}}\right\}\right) \leq 1 / 2 \quad(s=0,1)
$$

Thus, by Proposition 2.2, $\left(I-H_{\lambda} \mathcal{U}(\lambda)\right)^{-1}=I+\sum_{j=1}^{\infty}\left(H_{\lambda} \mathcal{U}(\lambda)\right)^{j}$ exists in $\mathcal{L}\left(\mathcal{X}_{q}\left(\mathbb{R}_{+}^{N}\right)\right)$ and satisfies the estimate:

$$
\left.\mathcal{R}_{\mathcal{L}\left(\mathcal{X}_{q}\left(\mathbb{R}_{+}^{N}\right)\right)}\left(\left\{\left(\tau \partial_{\tau}\right)^{s}\left(I-H_{\lambda} \mathcal{U}(\lambda)\right)^{-1}\right) \mid \lambda \in \Sigma_{\vartheta, \lambda_{1}}\right\}\right) \leq 2 \quad(s=0,1) .
$$

Recall the definition of space $\mathbb{G}_{q}\left(\mathbb{R}_{+}^{N}\right)$ and its norm $\|\cdot\|_{\mathbb{G}_{q}\left(\mathbb{R}_{+}^{N}\right)}$ given in (1-7) with $D=\mathbb{R}_{+}^{N}$. For any $\lambda \neq 0$, there exists a positive constant $c_{\lambda}$ depending on $\lambda$ such that

$$
c_{\lambda}^{-1}\left\|H_{\lambda}(F, G)\right\|_{\mathcal{X}_{q}\left(\mathbb{R}_{+}^{N}\right)} \leq\|(F, G)\|_{\mathbb{G}_{q}\left(\mathbb{R}_{+}^{N}\right)} \leq c_{\lambda}\left\|H_{\lambda}(F, G)\right\|_{\mathcal{X}_{q}\left(\mathbb{R}_{+}^{N}\right)},
$$

i.e., $\left\|H_{\lambda}(F, G)\right\|_{\mathcal{X}_{q}\left(\mathbb{R}_{+}^{N}\right)}$ is an equivalent norm of $\mathbb{G}_{q}\left(\mathbb{R}_{+}^{N}\right)$. By $(5-17)$,

$$
\left\|H_{\lambda} \mathcal{U}(\lambda) H_{\lambda}(F, G)\right\|_{\mathcal{X}_{q}\left(\mathbb{R}_{+}^{N}\right)} \leq \frac{1}{2}\left\|H_{\lambda}(F, G)\right\|_{\mathcal{X}_{q}\left(\mathbb{R}_{+}^{N}\right)}
$$

which yields that $\left(I-\mathcal{U}(\lambda) H_{\lambda}\right)^{-1}=I+\sum_{j=1}^{\infty}\left(\mathcal{U}(\lambda) H_{\lambda}\right)^{j}$ exists in $\mathcal{L}\left(\mathbb{G}_{q}\left(\mathbb{R}_{+}^{N}\right)\right)$ for any $\lambda \in \Sigma_{\vartheta, \lambda_{1}}$. In view of $(5-15), U:=\mathcal{T}(\lambda) H_{\lambda}\left(I-\mathcal{U}(\lambda) H_{\lambda}\right)^{-1}(F, G)$ satisfies the equation

$$
L_{\lambda}(D) U=\left(I-\mathcal{U}(\lambda) H_{\lambda}\right)\left(I-\mathcal{U}(\lambda) H_{\lambda}\right)^{-1}(F, G)=(F, G),
$$

that is, $U$ satisfies equations (5-13). Thus, we define the operator $\mathcal{V}(\lambda)$ by

$$
\mathcal{V}(\lambda)=\mathcal{T}(\lambda)\left(I-H_{\lambda} \mathcal{U}(\lambda)\right)^{-1},
$$

we obtain $U=\mathcal{V}(\lambda) H_{\lambda}(F, G)$. In fact, noting that

$$
\left(I-H_{\lambda} \mathcal{U}(\lambda)\right)^{-1} H_{\lambda}=H_{\lambda}+\sum_{j=1}^{\infty}\left(H_{\lambda} \mathcal{U}(\lambda)\right)^{j} H_{\lambda}=H_{\lambda}\left(I+\sum_{j=1}^{\infty}\left(\mathcal{U}(\lambda) H_{\lambda}\right)^{j}\right)=H_{\lambda}\left(I-\mathcal{U}(\lambda) H_{\lambda}\right)^{-1},
$$

we see that $\mathcal{V}(\lambda) H_{\lambda}(F, G)=\mathcal{T}(\lambda) H_{\lambda}\left(I-\mathcal{U}(\lambda) H_{\lambda}\right)^{-1}(F, G)=U$. Therefore, $U=\mathcal{V}(\lambda) H_{\lambda}(F, G)$ is the unique solution of the equations (5-13) for any $\lambda \in \Sigma_{\vartheta, \lambda_{1}}$ and $(F, G) \in \mathbb{G}_{q}\left(\mathbb{R}_{+}^{N}\right)$. Here, the uniqueness of the solution follows from the existence of solutions of the dual problem. (For this, see also Shibata and Shimizu [29, Proof of Theorem 4.3] for a similar argument.)

Setting $\mathcal{V}(\lambda)=\left(\mathcal{V}_{1}(\lambda), \lambda \mathcal{V}_{1}(\lambda), \mathcal{V}_{2}(\lambda)\right)^{\top}$, by (5-18), Theorem 3.1 and Proposition 2.2, we have

$$
\begin{gathered}
\mathcal{R}_{\mathcal{L}\left(\mathcal{X}_{q}\left(\mathbb{R}_{+}^{N}\right), H_{q}^{4-j}\left(\mathbb{R}_{+}^{N}\right)\right)}\left(\left\{\left(\tau \partial_{\tau}\right)^{s}\left(\lambda^{j / 2} \mathcal{V}_{1}(\lambda)\right) \mid \lambda \in \Sigma_{\vartheta, \lambda_{1}}\right\}\right) \leq C_{N, q, \lambda_{1}} \quad(s=0,1, j=0,1,2,3,4), \\
\mathcal{R}_{\mathcal{L}\left(\mathcal{X}_{q}\left(\mathbb{R}_{+}^{N}\right), H_{q}^{2-j}\left(\mathbb{R}_{+}^{N}\right)\right)}\left(\left\{\left(\tau \partial_{\tau}\right)^{s}\left(\lambda^{j / 2} \mathcal{V}_{2}(\lambda)\right) \mid \lambda \in \Sigma_{\vartheta, \lambda_{1}}\right\}\right) \leq C_{N, q, \lambda_{1}} \quad(s=0,1, \quad j=0,1,2) .
\end{gathered}
$$

Finally, defining $\mathcal{T}_{+i}(\lambda)(i=1,2)$ by

$$
\mathcal{T}_{+i}(\lambda)\left(F^{\prime}, G, G^{\prime}, g_{1}^{\prime \prime}\right)=\left(\mathcal{V}_{i}(\lambda)\left[\left(F^{\prime}, G, G^{\prime}, g_{1}^{\prime \prime}\right) \circ \Phi\right]\right) \circ \Phi^{-1},,
$$

we see that $\mathcal{T}_{+i}(\lambda)(i=1,2)$ are the operators satisfying the required properties in Theorem 5.1 , which completes the proof of Theorem 5.1.

\section{Proof of Theorem 1.4}

First of all, we state several properties of a uniform $C^{4}$-domain.

Proposition 6.1. Let $\Omega$ be a uniform $C^{4}$-domain in $\mathbb{R}^{N}$ with boundary $\Gamma$. Then, for any positive constant $M_{1}$, there exist constants $M_{2}>0, d \in(0,1)$, an open set $U$, at most countably many functions $\Phi_{j} \in H_{\infty}^{4}\left(\mathbb{R}^{N}\right)^{N} \cap C^{4}\left(\mathbb{R}^{N}, \mathbb{R}^{N}\right)$ and points $x_{j} \in \Gamma$ such that the following assertions hold:

(i) For every $j \in \mathbb{N}$, the $\operatorname{map} \mathbb{R}^{N} \ni x \mapsto \Phi_{j}(x) \in \mathbb{R}^{N}$ is bijective. 
(ii) $\Omega=U \cup \bigcup_{j=1}^{\infty}\left(\Phi_{j}\left(\mathbb{R}_{+}^{N}\right) \cap B_{d}\left(x_{j}\right)\right), \Phi_{j}\left(\mathbb{R}_{+}^{N}\right) \cap B_{d}\left(x_{j}\right)=\Omega \cap B_{d}\left(x_{j}\right)$, and $\Gamma \cap B_{d}\left(x_{j}\right)=\Phi_{j}\left(\mathbb{R}_{0}^{N}\right) \cap$ $B_{d}\left(x_{j}\right)$.

(iii) There exist $C^{\infty}$-functions $\zeta_{j}$ and $\tilde{\zeta}_{j}(j=0,1,2, \ldots)$ such that $\operatorname{supp} \zeta_{0}, \operatorname{supp} \tilde{\zeta}_{0} \subset G, \operatorname{supp} \zeta_{j}, \operatorname{supp} \tilde{\zeta}_{j} \subset B_{d}\left(x_{j}\right),\left\|\zeta_{j}\right\|_{H_{\infty}^{4}\left(\mathbb{R}^{N}\right)} \leq c_{0},\left\|\tilde{\zeta}_{j}\right\|_{H_{\infty}^{4}\left(\mathbb{R}^{N}\right)} \leq c_{0}$, $\tilde{\zeta}_{j}=1$ on $\operatorname{supp} \zeta_{j}, \sum_{j=0}^{\infty} \zeta_{j}=1$ on $\bar{\Omega}, \sum_{j=1}^{\infty} \zeta_{j}=1$ on $\Gamma$.

Here, $c_{0}$ is a constant which depends on $M_{2}, N, q, q^{\prime}$ and $r$, but is independent of $j=0,1,2, \ldots$

(iv) $\nabla \Phi_{j}=\mathcal{R}_{j}+R_{j}, \nabla\left(\Phi_{j}\right)^{-1}=\mathcal{R}_{-j}+R_{-j}$, where $\mathcal{R}_{j}$ and $\mathcal{R}_{-j}$ are $N \times N$ constant orthogonal matrices, and $R_{j}$ and $R_{-j}$ are $N \times N$ matrices of $H_{\infty}^{3}$-functions defined on $\mathbb{R}^{N}$ which satisfy the conditions: $\left\|R_{j}\right\|_{L_{\infty}\left(\mathbb{R}^{N}\right)} \leq M_{1},\left\|R_{-j}\right\|_{L_{\infty}\left(\mathbb{R}^{N}\right)} \leq M_{1},\left\|\nabla R_{j}\right\|_{H_{\infty}^{2}\left(\mathbb{R}^{N}\right)} \leq M_{2}$ and $\left\|\nabla R_{-j}\right\|_{H_{\infty}^{2}\left(\mathbb{R}^{N}\right)} \leq$ $M_{2}$ for any $j=1,2, \ldots$.

(v) There exists a natural number $L \geq 2$ such that any $L+1$ distinct sets of $\left\{U_{j} \mid j=0,1,2, \ldots\right\}$ with $U_{0}=U$ and $U_{j}=\Phi_{j}\left(B_{d}(0)\right)(j=1,2,3, \ldots)$ have an empty intersection.

In what follows, we write $\Omega_{\ell}=\Phi_{\ell}\left(\mathbb{R}_{+}^{N}\right)$, and $\Gamma_{\ell}=\Phi_{\ell}\left(\mathbb{R}_{0}^{N}\right)$. For $f \in L_{q}(\Omega)$, we have

$$
\sum_{\ell=1}^{\infty}\left\|\tilde{\zeta}_{\ell} f\right\|_{L_{q}\left(\Omega_{\ell}\right)}^{q}+\left\|\tilde{\zeta}_{0} f\right\|_{L_{q}\left(\mathbb{R}^{N}\right)}^{q} \leq C_{q}\|f\|_{L_{q}(\Omega)}^{q} .
$$

For $(F, G) \in \mathbb{G}_{q}(\Omega)$, let $U_{0}$ and $U_{\ell}$ be solutions of the equations:

$$
\begin{aligned}
& \lambda U_{0}-A(D) U_{0}=\tilde{\zeta}_{0} F \quad \text { in } \mathbb{R}^{N}, \\
& \lambda U_{\ell}-A(D) U_{\ell}=\tilde{\zeta}_{\ell} F \quad \text { in } \Omega_{\ell}, \quad B(D) U_{\ell}=\tilde{\zeta}_{\ell} G \quad \text { on } \Gamma_{\ell} .
\end{aligned}
$$

By Theorem 2.3 and Theorem 5.1, there exist operator families $\mathcal{S}_{01}(\lambda), \mathcal{S}_{02}(\lambda), \mathcal{S}_{\ell 1}(\lambda)$ and $\mathcal{S}_{\ell 2}(\lambda)$ with

$$
\begin{array}{lll}
\mathcal{S}_{01}(\lambda) \in \mathcal{C}\left(\Sigma_{\vartheta, \lambda_{0}}, \mathcal{L}\left(L_{q}\left(\mathbb{R}^{N}\right)^{2}, H_{q}^{4}\left(\mathbb{R}^{N}\right)\right)\right), & \mathcal{S}_{02}(\lambda) \in \mathcal{C}\left(\Sigma_{\vartheta, \lambda_{0}}, \mathcal{L}\left(L_{q}\left(\mathbb{R}^{N}\right)^{2}, H_{q}^{2}\left(\mathbb{R}^{N}\right)\right)\right), \\
\mathcal{S}_{\ell 1}(\lambda) \in \mathcal{C}\left(\Sigma_{\vartheta, \lambda_{0}}, \mathcal{L}\left(L_{q}\left(\Omega_{\ell}\right)^{2}, H_{q}^{4}\left(\Omega_{\ell}\right)\right)\right), & \mathcal{S}_{\ell 2}(\lambda) \in \mathcal{C}\left(\Sigma_{\vartheta, \lambda_{0}}, \mathcal{L}\left(L_{q}\left(\Omega_{\ell}\right)^{2}, H_{q}^{2}\left(\Omega_{\ell}\right)\right)\right)
\end{array}
$$

such that

$$
\begin{aligned}
U_{0} & =\left(\mathcal{S}_{01}(\lambda) \tilde{\zeta}_{0} F^{\prime}, \lambda \mathcal{S}_{01}(\lambda) \tilde{\zeta}_{0} F^{\prime}, \mathcal{S}_{02}(\lambda) \tilde{\zeta}_{0} F^{\prime}\right)^{\top}, \\
U_{\ell} & =\left(\mathcal{S}_{\ell 1}(\lambda) H_{\lambda}\left(\tilde{\zeta}_{\ell} F, \tilde{\zeta}_{\ell} G\right), \lambda \mathcal{S}_{\ell 1}(\lambda) H_{\lambda}\left(\tilde{\zeta}_{\ell} F, \tilde{\zeta}_{\ell} G\right), \mathcal{S}_{\ell 2}(\lambda) H_{\lambda}\left(\tilde{\zeta}_{\ell} F, \tilde{\zeta}_{\ell} G\right)\right)^{\top}
\end{aligned}
$$

uniquely solve (6-2) with $\lambda \in \Sigma_{\vartheta, \lambda_{0}}$ and (6-3) with $\lambda \in \Sigma_{\vartheta, \lambda_{1}}$, respectively. Here and hereafter, $H_{\lambda}$ is an operator acting on $(F, G)$ defined by $H_{\lambda}(F, G)=\left(f_{1}, f_{2}, G, \lambda^{1 / 2} G, \lambda g_{1}\right)$, where $F=\left(0, f_{1}, f_{2}\right)$ and $G=\left(g_{1}, g_{2}, g_{3}\right)$. Moreover, we have

$$
\begin{aligned}
& \mathcal{R}_{\mathcal{L}\left(L_{q}\left(\mathbb{R}^{N}\right)^{2}, H_{q}^{4-j}\left(\mathbb{R}^{N}\right)\right)}\left(\left\{\left(\tau \partial_{\tau}\right)^{s}\left(\lambda^{j / 2} \mathcal{S}_{01}(\lambda)\right) \mid \lambda \in \Sigma_{\vartheta, \lambda_{0}}\right\}\right) \leq \kappa_{1} \quad(j=0,1,2,3,4) ; \\
& \mathcal{R}_{\left.L_{q}\left(\mathbb{R}^{N}\right)^{2}, H_{q}^{2-j}\left(\mathbb{R}^{N}\right)\right)}\left(\left\{\left(\tau \partial_{\tau}\right)^{s}\left(\lambda^{j / 2} \mathcal{S}_{02}(\lambda)\right) \mid \lambda \in \Sigma_{\vartheta, \lambda_{0}}\right\}\right) \leq \kappa_{1} \quad(j=0,1,2) ; \\
& \mathcal{R}_{\mathcal{L}\left(\mathcal{X}_{q}\left(\Omega_{\ell}\right), H_{q}^{4-j}\left(\Omega_{\ell}\right)\right)}\left(\left\{\left(\tau \partial_{\tau}\right)^{s}\left(\lambda^{j / 2} \mathcal{S}_{\ell 1}(\lambda)\right) \mid \lambda \in \Sigma_{\vartheta, \lambda_{1}}\right\}\right) \leq \kappa_{1} \quad(j=0,1,2,3,4), \\
& \mathcal{R}_{\mathcal{L}\left(\mathcal{X}_{q}\left(\Omega_{\ell}\right), H_{q}^{2-j}\left(\Omega_{\ell}\right)\right)}\left(\left\{\left(\tau \partial_{\tau}\right)^{s}\left(\lambda^{j / 2} \mathcal{S}_{\ell 2}(\lambda)\right) \mid \lambda \in \Sigma_{\vartheta, \lambda_{1}}\right\}\right) \leq \kappa_{1} \quad(j=0,1,2),
\end{aligned}
$$

for $s=0,1$ with some constant $\kappa_{1}$ independent of $\ell$. We may assume that $0 \leq \lambda_{0} \leq \lambda_{1}$. By (6-7),

$$
\begin{aligned}
& \sum_{j=0}^{4}\left\|\lambda^{j / 2} u_{0}\right\|_{H_{q}^{4-j}\left(\mathbb{R}^{N}\right)}+\sum_{j=0}^{2}\left\|\lambda^{j / 2}\left(v_{0}, \theta_{0}\right)\right\|_{H_{q}^{2-j}\left(\mathbb{R}^{N}\right)} \leq \kappa_{1}\left\|\left(\tilde{\zeta}_{0} f_{1}, \tilde{\zeta}_{0} f_{2}\right)\right\|_{L_{q}\left(\mathbb{R}^{N}\right)}, \\
& \sum_{j=0}^{4}\left\|\lambda^{j / 2} u_{\ell}\right\|_{H_{q}^{4-j}\left(\Omega_{\ell}\right)}+\sum_{j=0}^{2}\left\|\lambda^{j / 2}\left(v_{\ell}, \theta_{\ell}\right)\right\|_{H_{q}^{2-j}\left(\Omega_{\ell}\right)} \leq \kappa_{1}\left\|\left(\tilde{\zeta}_{\ell} f_{1}, \tilde{\zeta}_{\ell} f_{2}, \tilde{\zeta}_{\ell} G, \lambda^{1 / 2} \tilde{\zeta}_{\ell} G, \lambda \tilde{\zeta}_{\ell} g_{1}\right)\right\|_{\mathcal{X}_{q}\left(\Omega_{\ell}\right)}
\end{aligned}
$$


where we have set $U_{0}=\left(u_{0}, v_{0}, \theta_{0}\right)^{\top}$ and $U_{\ell}=\left(u_{\ell}, v_{\ell}, \theta_{\ell}\right)^{\top}$. For any $\left(F^{\prime}, G, G^{\prime}, g_{1}^{\prime \prime}\right) \in \mathcal{X}_{q}(\Omega)$ and $\lambda \in \Sigma_{\vartheta, \lambda_{1}}$, we define an operator $\mathcal{A}_{i}(\lambda)$ acting on $\left(F^{\prime}, G, G^{\prime}, g_{1}^{\prime \prime}\right)$ by

$$
\mathcal{A}_{i}(\lambda)\left(F, G, G^{\prime}, g_{1}^{\prime \prime}\right)=\zeta_{0} \mathcal{S}_{0 i}(\lambda)\left(\tilde{\zeta}_{0} F^{\prime}\right)+\sum_{\ell=1}^{\infty} \zeta_{\ell} \mathcal{S}_{\ell i}(\lambda)\left(\tilde{\zeta}_{\ell} F^{\prime}, \tilde{\zeta}_{\ell} G, \tilde{\zeta}_{\ell} G^{\prime}, \tilde{\zeta}_{\ell} g_{1}^{\prime \prime}\right) \quad(i=1,2) .
$$

By (6-1) and (6-7),

$$
\begin{aligned}
& \mathcal{R}_{\mathcal{L}\left(\mathcal{X}_{q}(\Omega), H_{q}^{4-j}(\Omega)\right)}\left(\left\{\left(\tau \partial_{\tau}\right)^{s}\left(\lambda^{j / 2} \mathcal{A}_{1}(\lambda)\right) \mid \lambda \in \Sigma_{\vartheta, \lambda_{1}}\right\}\right) \leq C_{q} \kappa_{1} \quad(s=0,1, \quad j=0,1,2,3,4), \\
& \mathcal{R}_{\mathcal{L}\left(\mathcal{X}_{q}(\Omega), H_{q}^{2-j}(\Omega)\right)}\left(\left\{\left(\tau \partial_{\tau}\right)^{s}\left(\lambda^{j / 2} \mathcal{A}_{2}(\lambda)\right) \mid \lambda \in \Sigma_{\vartheta, \lambda_{1}}\right\}\right) \leq C_{q} \kappa_{1} \quad(s=0,1, \quad j=0,1,2) .
\end{aligned}
$$

For any $C^{\infty}$-function $\zeta$, we define operators $A_{R}(\zeta, D)$ and $B_{R}(\zeta, D)$ acting on $U$ by

$$
A_{R}(\zeta, D) U:=A(D)(\zeta U)-\zeta A(D) U, \quad B_{R}(\zeta, D) U:=B(D)(\zeta U)-\zeta B(D) U
$$

and then we have

$$
A_{R}(\zeta, D)=\left(0, A_{R 2}(\zeta, D) U, A_{R 3}(\zeta, D) U\right)^{\top}, \quad B_{R}(\zeta, D) U=\left(B_{R 1}(\zeta, D) U, B_{R 2}(\zeta, D) U, B_{R 3}(\zeta, D) U\right)^{\top}
$$

with

$$
\begin{aligned}
& A_{R 2}(\zeta, D) U=-\{\Delta((\Delta \zeta) u+2(\nabla \zeta) \cdot(\nabla u))+(\Delta \zeta)(\Delta u)+2(\nabla \zeta) \cdot(\nabla \Delta u)+(\Delta \zeta) \theta+2(\nabla \zeta) \cdot(\nabla \theta)\}, \\
& A_{R 3}(\zeta, D) U=(\Delta \zeta) v+2(\nabla \zeta) \cdot(\nabla v)+(\Delta \zeta) \theta+2(\nabla \zeta) \cdot(\nabla \theta), \\
& B_{R 1}(\zeta, D) U=2(\nabla \zeta) \cdot(\nabla u)+(\Delta \zeta) u-(1-\beta)\left(2\left(\nabla^{\prime} \zeta\right) \cdot\left(\nabla^{\prime} u\right)+\left(\Delta^{\prime} \zeta\right) u\right), \\
& B_{R 2}(\zeta, D) U=\partial_{\nu}\left\{2(\nabla \zeta) \cdot(\nabla u)+(\Delta \zeta) u+(1-\beta)\left(2\left(\nabla^{\prime} \zeta\right) \cdot\left(\nabla^{\prime} u\right)+\left(\Delta^{\prime} \zeta\right) u\right)\right\}+\left(\partial_{\nu} \zeta\right)\left(\Delta+(1-\beta) \Delta^{\prime}\right) u, \\
& B_{R 3}(\zeta, D) U=\left(\partial_{\nu} \zeta\right) \theta,
\end{aligned}
$$

where $U=(u, v, \theta)^{\top}$. Let $\mathcal{A}(\lambda)=\left(\mathcal{A}_{1}(\lambda), \lambda \mathcal{A}_{1}(\lambda), \mathcal{A}_{2}(\lambda)\right)^{\top}$ and then by (6-2), (6-3) and (6-6), we see that $U=\mathcal{A}(\lambda) H_{\lambda}(F, G)$ satisfies the equation:

$$
\lambda U-A(D) U=F-\mathbb{U}_{1}(\lambda)(F, G) \quad \text { in } \Omega, \quad B(D) U=G-\mathbb{U}_{2}(\lambda)(F, G) \quad \text { on } \Gamma,
$$

where

$$
\begin{aligned}
& \mathbb{U}_{1}(\lambda)(F, G)=A_{R}\left(\zeta_{0}, D\right) \mathcal{S}_{0}(\lambda)\left(\tilde{\zeta}_{0} F^{\prime}\right)+\sum_{\ell=1}^{\infty} A_{R}\left(\zeta_{\ell}, D\right) \mathcal{S}_{\ell}(\lambda) H_{\lambda}\left(\tilde{\zeta}_{\ell} F, \tilde{\zeta}_{\ell} G\right), \\
& \mathbb{U}_{2}(\lambda)(F, G)=\sum_{\ell=1}^{\infty} B_{R}\left(\zeta_{\ell}, D\right) \mathcal{S}_{\ell}(\lambda) H_{\lambda}\left(\tilde{\zeta}_{\ell} F, \tilde{\zeta}_{\ell} G\right) .
\end{aligned}
$$

Setting $L_{\lambda}(D)=(\lambda I-A(D), B(D))$ and $\mathbb{U}(\lambda)(F, G)=\left(\mathbb{U}_{1}(\lambda)(F, G), \mathbb{U}_{2}(\lambda)(F, G)\right)$, we have

$$
L_{\lambda}(D) \mathcal{A}(\lambda) H_{\lambda}(F, G)=(I-\mathbb{U}(\lambda))(F, G) \quad \text { on } \Omega \times \Gamma .
$$

By (6-2) and (6-3), we have

$$
H_{\lambda} \mathbb{U}(\lambda)(F, G)=\left(\begin{array}{c}
A_{R}\left(\zeta_{0}, D\right) \mathcal{S}_{0}(\lambda)\left(\tilde{\zeta}_{0} F^{\prime}\right) \\
0 \\
0 \\
0
\end{array}\right)+\sum_{\ell=1}^{\infty}\left(\begin{array}{c}
A_{R}\left(\zeta_{\ell}, D\right) \mathcal{S}_{\ell}(\lambda) H_{\lambda}\left(\tilde{\zeta}_{\ell} F, \tilde{\zeta}_{\ell} G\right) \\
B_{R}\left(\zeta_{\ell}, D\right) \mathcal{S}_{\ell}(\lambda) H_{\lambda}\left(\tilde{\zeta}_{\ell} F, \tilde{\zeta}_{\ell} G\right) \\
\lambda^{1 / 2} B_{R}\left(\zeta_{\ell}, D\right) \mathcal{S}_{\ell}(\lambda) H_{\lambda}\left(\tilde{\zeta}_{\ell} F, \tilde{\zeta}_{\ell} G\right) \\
\lambda B_{R 1}\left(\zeta_{\ell}, D\right)\left(\mathcal{S}_{\ell 1}(\lambda) H_{\lambda}\left(\tilde{\zeta}_{\ell} F, \tilde{\zeta}_{\ell} G\right)\right.
\end{array}\right)
$$

where $F=\left(0, f_{1}, f_{2}\right)^{\top}$ and $F^{\prime}=\left(f_{1}, f_{2}\right)^{\top}$. We have

$$
H_{\lambda} \mathbb{U}(\lambda)(F, G)=\mathcal{U}(\lambda) H_{\lambda}(F, G)
$$


with

$$
\mathcal{U}(\lambda)\left(F^{\prime}, G, G^{\prime}, g_{1}^{\prime \prime}\right)=\left(\begin{array}{c}
A_{R}\left(\zeta_{0}, D\right) \mathcal{S}_{0}(\lambda)\left(\tilde{\zeta}_{0} F^{\prime}\right) \\
0 \\
0 \\
0
\end{array}\right)+\sum_{\ell=1}^{\infty}\left(\begin{array}{c}
A_{R}\left(\zeta_{\ell}, D\right) \mathcal{S}_{\ell}(\lambda)\left(\tilde{\zeta}_{\ell} F^{\prime}, \tilde{\zeta}_{\ell} G, \tilde{\zeta}_{\ell} G^{\prime}, \tilde{\zeta}_{\ell} g_{1}^{\prime \prime}\right) \\
B_{R}\left(\zeta_{\ell}, D\right) \mathcal{S}_{\ell}(\lambda)\left(\tilde{\zeta}_{\ell} F^{\prime}, \tilde{\zeta}_{\ell} G, \tilde{\zeta}_{\ell} G^{\prime}, \tilde{\tilde{\zeta}}_{\ell} g_{1}^{\prime \prime}\right) \\
\lambda^{1 / 2} B_{R}\left(\zeta_{\ell}, D\right) \mathcal{S}_{\ell}(\lambda)\left(\tilde{\zeta}_{\ell} F^{\prime}, \tilde{\zeta}_{\ell} G, \tilde{\zeta}_{\ell} G^{\prime}, \tilde{\zeta}_{\ell} g_{1}^{\prime \prime}\right) \\
\lambda B_{R 1}\left(\zeta_{\ell}, D\right)\left(\mathcal{S}_{\ell 1}(\lambda)\left(\tilde{\zeta}_{\ell} F^{\prime}, \tilde{\zeta}_{\ell} G, \tilde{\zeta}_{\ell} G^{\prime}, \tilde{\zeta}_{\ell} g_{1}^{\prime \prime}\right)\right.
\end{array}\right) .
$$

By (6-1), (6-7) and Proposition 2.2, we have

$$
\mathcal{R}_{\mathcal{L}\left(\mathcal{X}_{q}(\Omega)\right)}\left(\left\{\left(\tau \partial_{\tau}\right)^{s} \mathcal{U}(\lambda) \mid \lambda \in \Sigma_{\vartheta, \lambda_{2}}\right\}\right) \leq C_{q} \kappa_{1} \lambda_{2}^{-1 / 2} \quad(s=0,1)
$$

for any $\lambda_{2} \geq \lambda_{1}$. We choose $\lambda_{2}$ so large that

$$
C_{q} \kappa_{1} \lambda_{2}^{-1 / 2} \leq 1 / 2
$$

By (6-13), (6-14) and (6-15), we have

$$
\left\|H_{\lambda} \mathbb{U}(\lambda)(F, G)\right\|_{\mathcal{X}_{q}(\Omega)} \leq \frac{1}{2}\left\|H_{\lambda}(F, G)\right\|_{\mathcal{X}_{q}(\Omega)} .
$$

By the equivalence of the norms $\left\|H_{\lambda}(\cdot)\right\|_{\mathcal{X}_{q}(\Omega)}$ and $\|\cdot\|_{\mathbb{G}_{q}(\Omega)}$ (cf. (5-19)), the inverse $(I-\mathbb{U}(\lambda))^{-1}=$ $I+\sum_{n=1}^{\infty} \mathbb{U}(\lambda)^{n}$ exists in $\mathcal{L}\left(\mathbb{G}_{q}(\Omega)\right)$. Let $V=\mathcal{A}(\lambda) H_{\lambda}(I-\mathbb{U}(\lambda))^{-1}(F, G)$, and then by (6-12) $V$ solves the equation:

$$
L_{\lambda}(D) V=(F, G) \quad \text { on } \Omega \times \Gamma .
$$

The uniqueness of solutions follows from the existence of solutions of the dual problem, so that $V$ is the unique solution of the equation (6-17).

On the other hand, by (6-14) and (6-15), $I-\mathcal{U}(\lambda)=I+\sum_{n=1}^{\infty} \mathcal{U}(\lambda)^{n}$ exists in $\mathcal{C}\left(\Sigma_{\vartheta, \lambda_{2}}, \mathcal{L}\left(\mathcal{X}_{q}(\Omega)\right)\right)$ and satisfies the estimate:

$$
\mathcal{R}_{\mathcal{L}\left(\mathcal{X}_{q}(\Omega)\right)}\left(\left\{\left(\tau \partial_{\tau}\right)^{s}(I-\mathcal{U}(\lambda))^{-1} \mid \lambda \in \Sigma_{\vartheta, \lambda_{2}}\right\}\right) \leq 2 \quad(s=0,1) .
$$

Moreover, by (6-13),

$$
H_{\lambda}(I-\mathbb{U}(\lambda))^{-1}=H_{\lambda}+\sum_{n=1}^{\infty} H_{\lambda} \mathbb{U}(\lambda)^{n}=\left(I+\sum_{n=1}^{\infty} \mathcal{U}(\lambda)^{n}\right) H_{\lambda}=(I-\mathcal{U}(\lambda))^{-1} H_{\lambda}
$$

Let $\mathcal{B}_{i}(\lambda)=\mathcal{A}_{i}(\lambda)(I-\mathcal{U}(\lambda))^{-1}(i=1,2)$. Then, by (6-9), (6-18) and Proposition 2.2,

$$
\begin{aligned}
& \mathcal{R}_{\mathcal{L}\left(\mathcal{X}_{q}(\Omega), H_{q}^{4-j}(\Omega)\right)}\left(\left\{\left(\tau \partial_{\tau}\right)^{s}\left(\lambda^{j / 2} \mathcal{B}_{1}(\lambda)\right) \mid \lambda \in \Sigma_{\vartheta, \lambda_{1}}\right\}\right) \leq C_{q} \kappa_{1} \quad(s=0,1, \quad j=0,1,2,3,4), \\
& \mathcal{R}_{\mathcal{L}\left(\mathcal{X}_{q}(\Omega), H_{q}^{2-j}(\Omega)\right)}\left(\left\{\left(\tau \partial_{\tau}\right)^{s}\left(\lambda^{j / 2} \mathcal{B}_{2}(\lambda)\right) \mid \lambda \in \Sigma_{\vartheta, \lambda_{1}}\right\}\right) \leq C_{q} \kappa_{1} \quad(s=0,1, \quad j=0,1,2) .
\end{aligned}
$$

Moreover, setting $\mathcal{B}(\lambda)=\left(\mathcal{B}_{1}(\lambda), \lambda \mathcal{B}_{1}(\lambda), \mathcal{B}_{2}(\lambda)\right)^{\top}$, by (6-19) we see that

$$
\mathcal{B}(\lambda) H_{\lambda}(F, G)=\mathcal{A}(\lambda) H_{\lambda}(I-\mathbb{U}(\lambda))^{-1}(F, G),
$$

and therefore $V=\mathcal{B}(\lambda) H_{\lambda}(F, G)$ is the unique solution of the equation (1-5) (cf. (6-17)). This completes the proof of Theorem 1.4 replacing $\lambda_{0}$ by $\lambda_{2}$.

\section{Proof of Theorem 1.3}

To prove Theorem 1.3, we start with

Lemma 7.1. Let $1<p, q<\infty$. For any $\theta_{0} \in B_{q, p}^{2-2 / p}(\Omega), u_{0} \in B_{q, p}^{4-2 / p}(\Omega)$, and $u_{1} \in B_{q, p}^{2-2 / p}(\Omega)$, there exist $\omega$ and $w$ such that $\left.\omega\right|_{t=0}=\theta_{0},\left.w\right|_{t=0}=u_{0}$, and $\left.\partial_{t} w\right|_{t=0}=u_{1}$ in $\Omega$,

$$
\omega \in \bigcap_{\ell=0}^{1} H_{p}^{\ell}\left((0, \infty), H_{q, p}^{2-2 \ell}(\Omega)\right), \quad w \in \bigcap_{\ell=0}^{2} H_{p}^{\ell}\left((0, \infty), H_{q}^{4-2 \ell}(\Omega),\right.
$$


and

$$
\begin{aligned}
& \sum_{\ell=0}^{1}\left\|\partial_{t}^{\ell} \omega\right\|_{L_{p}\left((0, \infty), H_{q}^{2-2 \ell}(\Omega)\right.} \leq C\left\|\theta_{0}\right\|_{B_{q, p}^{2-2 / p}(\Omega)}, \\
& \sum_{\ell=0}^{2}\left\|\partial_{t}^{\ell} w\right\|_{L_{p}\left((0, \infty), H_{q, p}^{4-2 \ell}(\Omega)\right.} \leq C\left(\left\|u_{0}\right\|_{B_{q}^{4-2 / p}(\Omega)}+\left\|u_{1}\right\|_{B_{q, p}^{2-2 / p}(\Omega)}\right) .
\end{aligned}
$$

with some positive constant $C$.

Proof. To prove the lemma, we consider the shifted heat equation:

$$
\partial_{t} v+v-\Delta v=f \quad \text { in }(0, \infty) \times \mathbb{R}^{N},\left.\quad v\right|_{t=0}=v_{0} .
$$

Employing the same argument as in the previous sections, it is easy to prove that for any $f \in L_{p}((0, \infty)$, $\left.L_{q}\left(\mathbb{R}^{N}\right)\right)$ and $v_{0} \in B_{q, p}^{2-2 / p}\left(\mathbb{R}^{N}\right)$, problem (7-1) admits a unique solution $v \in \bigcap_{\ell=0}^{1} H_{p}^{\ell}\left((0, \infty), H_{q}^{2-2 \ell}\left(\mathbb{R}^{N}\right)\right)$ possessing the estimate:

$$
\sum_{\ell=0}^{1}\left\|\partial_{t}^{\ell} v\right\|_{L_{p}\left((0, \infty), H_{q}^{2-2 \ell}\left(\mathbb{R}^{N}\right)\right)} \leq C\left(\left\|v_{0}\right\|_{B_{q, p}^{2-2 / p}\left(\mathbb{R}^{N}\right)}+\|f\|_{L_{p}\left((0, \infty), L_{q}(\Omega)\right)}\right) .
$$

Assume that $v_{0} \in B_{q, p}^{4-2 / p}\left(\mathbb{R}^{N}\right)$ and $f \in \bigcap_{\ell=0}^{1} H_{p}^{\ell}\left((0, \infty), H_{q}^{2-2 \ell}\left(\mathbb{R}^{N}\right)\right)$. Then, for any multi-index $\alpha \in \mathbb{N}_{0}^{N}$ with $|\alpha| \leq 2$ we have

$$
\partial_{t} \partial_{x}^{\alpha} v+\partial_{x}^{\alpha} v-\Delta \partial_{x}^{\alpha} v=\partial_{x}^{\alpha} f \quad \text { in }(0, \infty) \times \mathbb{R}^{N},\left.\quad \partial_{x}^{\alpha} v\right|_{t=0}=\partial_{x}^{\alpha} v_{0} .
$$

Thus, the unique existence of solutions of (7-1) yields that

$$
\begin{aligned}
& v \in \bigcap_{\ell=0}^{1} H_{p}^{\ell}\left((0, \infty), H_{q}^{4-2 \ell}\left(\mathbb{R}^{N}\right)\right), \\
& \sum_{\ell=0}^{1}\left\|\partial_{t}^{\ell} v\right\|_{L_{p}\left((0, \infty), H_{q}^{4-2 \ell}\left(\mathbb{R}^{N}\right)\right)} \leq C\left(\left\|v_{0}\right\|_{B_{q, p}^{4-2 / p}\left(\mathbb{R}^{N}\right)}+\|f\|_{L_{p}\left((0, \infty), H_{q}^{2}\left(\mathbb{R}^{N}\right)\right)}\right) .
\end{aligned}
$$

Moreover, the relation: $\partial_{t}^{2} v=-\partial_{t} v+\Delta \partial_{t} v+\partial_{t} f$ yields that $\partial_{t}^{2} v \in L_{p}\left((0, \infty), L_{q}\left(\mathbb{R}^{N}\right)\right)$ and

$$
\left\|\partial_{t}^{2} v\right\|_{L_{p}\left((0, \infty), L_{q}\left(\mathbb{R}^{N}\right)\right)} \leq C\left(\left\|v_{0}\right\|_{B_{q, p}^{4-2 / p}\left(\mathbb{R}^{N}\right)}+\sum_{\ell=0}^{1}\left\|\partial_{t}^{\ell} f\right\|_{L_{p}\left((0, \infty), H_{q}^{2-2 \ell}\left(\mathbb{R}^{N}\right)\right)}\right) .
$$

Let $\iota_{h}$ be an extension map as given in Introduction satisfying property (e-1). Let $\omega$ be a solution of the shifted heat equation:

$$
\partial_{t} \omega+\omega-\Delta \omega=0 \quad \text { in }(0, \infty) \times \mathbb{R}^{N},\left.\quad \omega\right|_{t=0}=\iota_{h} \theta_{0} .
$$

Since $\iota_{h} \theta_{0} \in B_{q, p}^{2-2 / p}\left(\mathbb{R}^{N}\right)$, by (7-2) with $f=0$ there exists a unique $\omega \in \bigcap_{\ell=0}^{1} H_{p}^{\ell}\left((0, \infty), H_{q}^{2-2 \ell}\left(\mathbb{R}^{N}\right)\right)$ satisfying the estimate

$$
\sum_{\ell=0}^{1}\left\|\partial_{t}^{\ell} \omega\right\|_{L_{p}\left((0, \infty), H_{q}^{2-2 \ell}\left(\mathbb{R}^{N}\right)\right)} \leq C\left\|\iota_{h} \theta_{0}\right\|_{B_{q, p}^{2-2 / p}\left(\mathbb{R}^{N}\right)} .
$$

Since $\iota_{h} \theta_{0}=\theta_{0}$ on $\Omega$ and $\left\|\iota_{h} \theta_{0}\right\|_{B_{q, p}^{2-2 / p}\left(\mathbb{R}^{N}\right)} \leq C\left\|\theta_{0}\right\|_{B_{q, p}^{2-2 / p}(\Omega)}$ with some constant $C>0$, the restriction of $\omega$ on $\Omega$ is the function satisfying the required properties.

Next, let $f$ be a solution of the shifted heat equation:

$$
\partial_{t} f+f-\Delta f=0 \quad \text { in }(0, \infty) \times \mathbb{R}^{N},\left.\quad f\right|_{t=0}=v_{0}
$$


with $v_{0}=\iota_{h} u_{1}+\iota_{h} u_{0}-\iota_{h} \Delta u_{0}$. Since $v_{0} \in B_{q, p}^{2-2 / p}\left(\mathbb{R}^{N}\right)$ and

$$
\left\|v_{0}\right\|_{B_{q, p}^{2-2 / p}\left(\mathbb{R}^{N}\right)} \leq C\left(\left\|u_{1}\right\|_{B_{q, p}^{2-2 / p}(\Omega)}+\left\|u_{0}\right\|_{B_{q, p}^{4-2 / p}(\Omega)}\right),
$$

there exists a unique $f \in \bigcap_{\ell=0}^{1} H_{p}^{\ell}\left((0, \infty), H_{q}^{2-2 \ell}\left(\mathbb{R}^{N}\right)\right)$ satisfying the estimate:

$$
\sum_{\ell=0}^{1}\left\|\partial_{t}^{\ell} f\right\|_{L_{p}\left((0, \infty), H_{q}^{2-2 \ell}\left(\mathbb{R}^{N}\right)\right)} \leq C\left(\left\|u_{1}\right\|_{B_{q, p}^{2-2 / p}(\Omega)}+\left\|u_{0}\right\|_{B_{q, p}^{4-2 / p}(\Omega)}\right) .
$$

Let $w$ be a solution of the shifted heat equation:

$$
\partial_{t} w+w-\Delta w=f \quad \text { in }(0, \infty) \times \mathbb{R}^{N},\left.\quad w\right|_{t=0}=\iota u_{0} .
$$

Then, by (7-3), (7-4) and (7-5), there exists a unique $w \in \bigcap_{\ell=0}^{2} H_{p}^{\ell}\left((0, \infty), H_{q}^{4-2 \ell}\left(\mathbb{R}^{N}\right)\right)$ satisfying the estimate:

$$
\sum_{\ell=0}^{2}\left\|\partial_{t}^{\ell} w\right\|_{L_{p}\left((0, \infty), H_{q}^{4-2 \ell}\left(\mathbb{R}^{N}\right)\right)} \leq C\left(\left\|u_{1}\right\|_{B_{q, p}^{2-2 / p}(\Omega)}+\left\|u_{0}\right\|_{B_{q, p}^{4-2 / p}(\Omega)}\right) .
$$

Moreover, by (7-6), $\left.\partial_{t} w\right|_{t=0}=v_{0}-\iota_{h} u_{0}+\Delta \iota_{h} u_{0}=\iota_{h} u_{1}$, so that the restriciton of $w$ on $\Omega$ satisfies the required properties, which completes the proof of Lemma 7.1.

In view of Lemma 7.1, to prove Theorem 1.3, it suffices to consider the equations (1-4) with $U_{0}=0$. Let $F=\left(0, f_{1}, f_{2}\right)^{\top}$ and $G=\left(g_{1}, g_{2}, g_{3}\right)$ satisfy the regularity condition:

$$
\left(f_{1}, f_{2}\right) \in L_{p}\left((0, T), L_{q}(\Omega)^{2}\right), \quad G \in H_{p}^{1}\left((0, T), L_{q}(\Omega) \times \mathbf{W}_{q}^{-1}(\Omega)^{2}\right) \cap L_{p}\left((0, T), H_{q}^{2}(\Omega) \times H_{q}^{1}(\Omega)^{2}\right)
$$

and the compatibility condition: $\left.G\right|_{t=0}=0$. In the following, given $f(t, \cdot)$ defined for $t \in[0, T], f_{0}(t, \cdot)$ denotes the zero extension of $f$ to $t<0$, that is, $f_{0}(t, \cdot)=f(t, \cdot)$ for $0<t<T$, and $f_{0}(t, \cdot)=0$ for $t<0$, and $E_{T} f$ denotes the extention of $f$ to $\mathbb{R}$ defined by

$$
\left[E_{T} f\right](t, \cdot)= \begin{cases}f_{0}(t, \cdot) & \text { for } t<T, \\ f_{0}(2 T-t, \cdot) & \text { for } t \geq T .\end{cases}
$$

Note that $E_{T} f$ vanishes for $t \notin[0,2 T]$ and moreover, if $\left.f\right|_{t=0}=0$, then

$$
\partial_{t}\left[E_{T} f\right](t, \cdot)= \begin{cases}\partial_{t} f(t, \cdot) & \text { for } t \leq T, \\ -\left(\partial_{t} f\right)(2 T-t, \cdot) & \text { for } t \geq T, \\ 0 & \text { for } t \notin[0,2 T] .\end{cases}
$$

Since $E_{T} f=f$ for $0 \leq t \leq T$, instead of the equations (1-4) with $U_{0}=0$, we consider the equations:

$$
U_{t}-A(D) U=E_{T} F \quad \text { in } \mathbb{R} \times \Omega, \quad B(D) U=E_{T} G \quad \text { on } \mathbb{R} \times \Gamma .
$$

Let $\mathcal{L}$ be the Laplace transform with respect to time variable $t$ and let $\mathcal{L}^{-1}$ be its inverse transform, which are defined by

$$
\begin{aligned}
\mathcal{L}[f](\lambda, \cdot) & =\int_{-\infty}^{\infty} e^{-\lambda t} f(t, \cdot) d t=\int_{\mathbb{R}} e^{-i \tau t} e^{-\gamma t} f(t, \cdot) d t, \\
\mathcal{L}^{-1}[f](t, \cdot) & =\frac{1}{2 \pi i} \int_{\gamma-i \infty}^{\gamma+i \infty} e^{\lambda t} f(\lambda, \cdot) d \lambda=\frac{e^{\gamma t}}{2 \pi} \int_{-\infty}^{\infty} f(\gamma+i \tau, \cdot) d \tau,
\end{aligned}
$$

where $\lambda=\gamma+i \tau \in \mathbb{C}$. Let $\mathcal{L}\left[E_{T} F\right](\lambda, \cdot)=J_{\lambda}$ and $\mathcal{L}\left[E_{T} G\right](\lambda, \cdot)=K_{\lambda}$. Since $E_{T} F$ and $E_{T} G$ vanish for $t \notin[0,2 T], J_{\lambda}$ and $K_{\lambda}$ are entire functions with respect to $\lambda \in \mathbb{C}$. Applying the Laplace transform to (7-9), we have

$$
\lambda V_{\lambda}-A(D) V_{\lambda}=J_{\lambda} \quad \text { in } \Omega, \quad B(D) V_{\lambda}=K_{\lambda} \quad \text { on } \Gamma,
$$


where we have set $V_{\lambda}=\mathcal{L}[U](\lambda, \cdot)$. Now, $V_{\lambda}=\mathcal{B}(\lambda) H_{\lambda}\left(J_{\lambda}, K_{\lambda}\right)$ is the unique solution of (7-10), so that the uniqueness of the solution yields that $V_{\lambda}$ is holomorphic for $\lambda \in \Sigma_{\vartheta, \lambda_{0}}$. Let $\mathcal{B}_{i}(\lambda)(i=1,2)$ be the operators given in Theorem 1.4 and set $\mathcal{B}(\lambda)=\left(\mathcal{B}_{1}(\lambda), \lambda \mathcal{B}_{1}(\lambda), \mathcal{B}_{2}(\lambda)\right)^{\top}$. Then, the unique solution $U$ of (7-9) is given by

$$
U(t, \cdot)=\mathcal{L}^{-1}\left[\mathcal{B}(\lambda) H_{\lambda}\left(J_{\lambda}, K_{\lambda}\right)\right]=e^{\gamma t} \mathcal{F}^{-1}\left[\mathcal{B}(\lambda) H_{\lambda}\left(\mathcal{F}\left[e^{-\gamma \cdot} E_{T} F\right], \mathcal{F}\left[e^{-\gamma \cdot} E_{T} G\right]\right)\right](t, \cdot) .
$$

Let $\Lambda^{1 / 2}$ be the operator defined by

$$
\left[\Lambda^{1 / 2} f\right](t, \cdot)=\mathcal{L}^{-1}\left[\lambda^{1 / 2} \mathcal{L}[f](\lambda, \cdot)\right](t),
$$

and let

$$
\left.\left\|e^{-\gamma t} f\right\|_{L_{p}(\mathbb{R}, X)}=\left\{\int_{-\infty}^{\infty} e^{-\gamma t}\|f(t, \cdot)\|_{X}\right)^{p} d t\right\}^{1 / p} .
$$

Since $|\lambda|^{1 / 2} \leq|\lambda|\left(1+|\xi|^{2}\right)^{-1 / 2}$ when $|\lambda| \geq 1+|\xi|^{2}$ and $|\lambda|^{1 / 2} \leq\left(1+|\xi|^{2}\right)^{1 / 2}$ when $|\lambda| \leq 1+|\xi|^{2}$, by the Bourgain theorem (cf. Proposition 2.2) we have

$$
\left\|e^{\gamma t} \Lambda^{1 / 2} f\right\|_{L_{p}\left(\mathbb{R}, L_{q}\left(\mathbb{R}^{N}\right)\right)} \leq C\left(\left\|e^{\gamma t} \partial_{t}(1-\Delta)^{-1 / 2} f\right\|_{L_{p}\left(\mathbb{R}, L_{q}\left(\mathbb{R}^{N}\right)\right)}+\left\|e^{\gamma t}(1-\Delta)^{1 / 2} f\right\|_{L_{p}\left(\mathbb{R}, L_{q}\left(\mathbb{R}^{N}\right)\right)}\right),
$$

so that by using property (e-2) of the extension map $\iota_{h}$ given in the introduction we have

$$
\left\|e^{\gamma t} \Lambda^{1 / 2} f\right\|_{L_{p}\left(\mathbb{R}, L_{q}(\Omega)\right)} \leq C\left(\left\|e^{\gamma t} \partial_{t} f\right\|_{L_{p}\left(\mathbb{R}, \mathbf{W}_{q}^{-1}(\Omega)\right)}+\left\|e^{\gamma t} f\right\|_{L_{p}\left(\mathbb{R}, H_{q}^{1}(\Omega)\right)}\right) .
$$

And also, using the extension map $\iota_{h}$ and Proposition 2.2, we have

$$
\begin{aligned}
& \left\|e^{-\gamma t} \partial_{t} f\right\|_{L_{p}\left(\mathbb{R}, H_{q}^{m}(\Omega)\right)}+\left\|e^{-\gamma t} f\right\|_{L_{p}\left(\mathbb{R}, H_{q}^{m+2}(\Omega)\right)} \\
& \left.\quad \leq C_{m, q}\left(\left\|e^{-\gamma t} \partial_{t} f\right\|_{L_{p}\left(\mathbb{R}, H_{q}^{m}(\Omega)\right)}+\left\|e^{-\gamma t} \Lambda^{1 / 2} \nabla f\right\|_{L_{p}\left(\mathbb{R}, H_{q}^{m}(\Omega)\right)}+\left\|e^{-\gamma t} \nabla^{2} f\right\|_{L_{p}\left(\mathbb{R}, H_{q}^{m}(\Omega)\right.}\right)\right)
\end{aligned}
$$

for any $m \in \mathbb{N} \cup\{0\}$. Therefore, using (7-11) and (7-12) and applying the Weis operator valued Fourier multiplier theorem with the help of Theorem 1.4, we have

$$
\begin{aligned}
\sum_{\ell=0}^{2} & \left\|e^{-\gamma t} \partial_{t}^{\ell} u\right\|_{L_{p}\left(\mathbb{R}, H_{q}^{4-2 \ell}(\Omega)\right)}+\sum_{\ell=0}^{1}\left\|e^{-\gamma t} \partial_{t}^{\ell} \theta\right\|_{L_{p}\left(\mathbb{R}, H_{q}^{2-2 \ell}(\Omega)\right)} \\
\leq & C\left(\left\|e^{-\gamma t} E_{T}\left(f_{1}, f_{2}\right)\right\|_{L_{p}\left(\mathbb{R}, L_{q}(\Omega)\right)}+\left\|e^{-\gamma t} E_{T} G\right\|_{L_{p}\left(\mathbb{R}, H_{q}^{2}(\Omega) \times H_{q}^{1}(\Omega)^{2}\right)}\right. \\
& \left.+\left\|e^{-\gamma t} \Lambda^{1 / 2} E_{T} G\right\|_{L_{p}\left(\mathbb{R}, H_{q}^{1}(\Omega) \times L_{q}(\Omega)^{2}\right)}+\left\|e^{-\gamma t} \partial_{t} E_{T} g_{1}\right\|_{L_{p}\left(\mathbb{R}, L_{q}(\Omega)\right)}\right) \\
\leq & C\left(\left\|e^{-\gamma t} E_{T}\left(f_{1}, f_{2}\right)\right\|_{L_{p}\left(\mathbb{R}, L_{q}(\Omega)\right)}+\left\|e^{-\gamma t} E_{T} G\right\|_{L_{p}\left(\mathbb{R}, H_{q}^{2}(\Omega) \times H_{q}^{1}(\Omega)^{2}\right)}\right. \\
& \left.+\left\|e^{-\gamma t} \partial_{t} E_{T} G\right\|_{L_{p}\left(\mathbb{R}, L_{q}(\Omega) \times \mathbf{W}_{q}^{-1}(\Omega)^{2}\right)}\right)
\end{aligned}
$$

for any $\gamma \geq \lambda_{0}$ with constant $C$ independent of $\gamma$, where we have set $U=\left(u, \partial_{t} u, \theta\right)$. Using (7-7) and (7-8) and noting that $\left.G\right|_{t=0}=0$, we have

$$
\begin{aligned}
\left\|e^{-\gamma t} E_{T}\left(f_{1}, f_{2}\right)\right\|_{L_{p}\left(\mathbb{R}, L_{q}(\Omega)\right)} & \leq C\left\|\left(f_{1}, f_{2}\right)\right\|_{L_{p}\left((0, T), L_{q}(\Omega)\right)}, \\
\left\|e^{-\gamma t} E_{T} G\right\|_{L_{p}\left(\mathbb{R}, H_{q}^{2}(\Omega) \times H_{q}^{1}(\Omega)^{2}\right)} & \leq C\|G\|_{L_{p}\left((0, T), H_{q}^{2}(\Omega) \times H_{q}^{1}(\Omega)^{2}\right)}, \\
\left\|e^{-\gamma t} \partial_{t} E_{T} G\right\|_{L_{p}\left(\mathbb{R}, L_{q}(\Omega) \times \mathbf{W}_{q}^{-1}(\Omega)^{2}\right)} & \leq C\left\|\partial_{t} G\right\|_{L_{p}\left((0, T), L_{q}(\Omega) \times \mathbf{W}_{q}^{-1}(\Omega)^{2}\right)},
\end{aligned}
$$

which, combined with (7-13), furnishes that

$$
\sum_{\ell=0}^{2}\left\|e^{-\gamma t} \partial_{t}^{\ell} u\right\|_{L_{p}\left(\mathbb{R}, H_{q}^{4-2 \ell}(\Omega)\right)}+\sum_{\ell=0}^{1}\left\|e^{-\gamma t} \theta\right\|_{L_{p}\left(\mathbb{R}, H_{q}^{2-2 \ell}(\Omega)\right)} \leq C I_{T}
$$

with

$$
I_{T}=\left\|e^{-\gamma t}\left(f_{1}, f_{2}\right)\right\|_{L_{p}\left((0, T), L_{q}(\Omega)\right)}+\|G\|_{L_{p}\left(\mathbb{R}, H_{q}^{2}(\Omega) \times H_{q}^{1}(\Omega)^{2}\right)}+\left\|\partial_{t} G\right\|_{L_{p}\left(\mathbb{R}, L_{q}(\Omega) \times \mathbf{W}_{q}^{-1}(\Omega)^{2}\right)^{2}} .
$$


Especially, for any $t<0$, we have

$$
\begin{aligned}
& e^{\gamma|t|} \sum_{\ell=0}^{2}\left\|\partial_{t}^{\ell} u\right\|_{L_{p}\left((-\infty, t), H_{q}^{4-2 \ell}(\Omega)\right)}+\sum_{\ell=0}^{1}\|\theta\|_{L_{p}\left((-\infty, t), H_{q}^{2-2 \ell}(\Omega)\right)} \\
& \leq \sum_{\ell=0}^{2}\left\|e^{-\gamma t} \partial_{t}^{\ell} u\right\|_{L_{p}\left(\mathbb{R}, H_{q}^{4-2 \ell}(\Omega)\right)}+\sum_{\ell=0}^{1}\left\|e^{-\gamma t} \theta\right\|_{L_{p}\left(\mathbb{R}, H_{q}^{2-2 \ell}(\Omega)\right)} \leq C I_{T}
\end{aligned}
$$

for any $\gamma \geq \lambda_{0}$, so that letting $\gamma \rightarrow \infty$, we have

$$
e^{\gamma|t|} \sum_{\ell=0}^{2}\left\|\partial_{t}^{\ell} u\right\|_{L_{p}\left((-\infty, t), H_{q}^{4-2 \ell}(\Omega)\right)}+\sum_{\ell=0}^{1}\|\theta\|_{L_{p}\left((-\infty, t), H_{q}^{2-2 \ell}(\Omega)\right)}=0
$$

for any $t<0$, which implies that $(u, \theta)=0$ for $t<0$.

Summing up, we have proved that $U=\left(u, \partial_{t} u, \theta\right)^{\top}$ satisifes the equations:

$$
U_{t}-A(D) U=F \quad \text { in } \Omega \times(0, T), \quad B(D) U=G \quad \text { on } \Gamma \times(0, T),\left.\quad U\right|_{t=0}=0 \quad \text { in } \Omega
$$

and the estimate:

$$
\sum_{\ell=0}^{2}\left\|e^{-\gamma t} \partial_{t}^{\ell} u\right\|_{L_{p}\left((0, T), H_{q}^{4-2 \ell}(\Omega)\right)}+\sum_{\ell=0}^{1}\left\|e^{-\gamma t} \theta\right\|_{L_{p}\left((0, T), H_{q}^{2-2 \ell}(\Omega)\right)} \leq C I_{T} .
$$

The uniqueness of the solutions follwos from the existence of solutions of the dual problem, which completes the proof of Theorem 1.3.

\section{References}

[1] J. Bourgain, Vector-valued singular integrals and the $H^{1}-B M O$ duality, In: Probability Theory and Harmonic Analysis, D. Borkholder (ed.) Marcel Dekker, New York (1986), 1-19.

[2] I. Chueshov and I. Lasiecka, Von Karman evolution equations, Springer Monographs in Mathematics, Springer, New York, 2010, Well-posedness and long-time dynamics.

[3] R. Denk, M. Hieber, and J. Prüss, $\mathcal{R}$-boundedness, Fourier multipliers and problems of elliptic and parabolic type, Mem. Amer. Math. Soc., 166(788), 2003.

[4] R. Denk and R. Racke, $L^{p}$-resolvent estimates and time decay for generalized thermoelastic plate equations, Electron. J. Differential Equations, No. 48, 16pp. (electronic), 2006.

[5] R. Denk, R. Racke, and Y. Shibata, $L_{p}$ theory for the linear thermoelastic plate equations in bounded and exterior domains, Adv. Differential Equations 14(7-8) (2009), 685-715.

[6] R. Denk, R. Racke, and Y. Shibata, Local energy decay estimate of soluions to the thermoelastic plate equations in two- and three-dimensional exterior domains, Z. Anal. Anwend., 29(1) (2010), 21-62.

[7] R. Denk and R. Schnaubelt, A structurally damped plate equations with Dirichlet-Neumann boundary conditions, J. Differential Equations, 259(4) (2015), 1323-1353.

[8] R. Denk and J. Seiler, On the maximal $L_{p}$-regularity of parabolic maixed-order system, J. Evol. Equ., 11(2) (2011), 371-404.

[9] Y. Enomoto and Y. Shibata, On the $\mathcal{R}$-sectoriality and the initial boundary value problem for the viscous compressible fluid flow, Funkcial. Ekvac., 56(3) (2013), 441-505.

[10] Y. Enomoto, L. von Below, and Y. Shibata, On some free boundary problem for a compressible barotropic viscous fluid flow, Ann. Univ. Ferrara Sez. VII Sci. Mat., 60(1) (2014), 55-89. 
[11] M. Girardi and L. Weis, Criteria for R-boundedness of operator families, In Evolution equations, volume 234 of Lecture Notes in Pure and Appl. Math., pages 203-221. Dekker, New York, 2003.

[12] J. U. Kim, On the energy decay of a linear thermoelastic bar and plate, SIAM J. Math. Anal., 23(4) (1992), 889-899.

[13] P. C. Kunstmann and L. Weis, Maximal $L_{p}$-regularity for parabolic equations, Fourier multiplier theorems and $H^{\infty}$-functional calculus, In Functional analytic methods for evolution equations, volume 1855 of Lecture Notes in Math., pages 65-311, Springer, Berlin, 2004.

[14] J. E. Lagnese, Boundary stabilization of thin plates, volume 10 of SIAM Studies in Applied Mathematics, Society for Industrial and Applied Mathematcs (SIAM), Philadelphia, PA, 1989

[15] I. Lasiecka and R. Triggiani, Analyticity, and lack thereof, of thermo-elastic semigroups, In Control and partial differential equations) Marseille-Luminy, 1997), volume 4 of ESAIM Proc., pages 199-222 (electropnic), Soc. Math. Appl. Indust., Paris,1998.

[16] I. Lasiecka and R. Triggiani, Analyticity of thermo-elastic semigroups with coupled hinged/Neumann B.C., Abstr. Appl. Anal., 3(1-2) (1998), 153-169.

[17] I. Lasiecka and M. Wilke, Maximal regularity and global existence of soluiotns to a quasilinear thermoelastic plate system, Discrete Contin. Dyn. Syst., 33(11-12) (2013), 5189-5202.

[18] K. Liu and Z. Liu, Exponential sstability and analyticity of abstract linear thermoelastic systems, Z. Angew. Math. Phys., 48(6) (1997), 885-904.

[19] Z. Liu and J. Yong, Qualitative properties of certain $C_{0}$ semigroups arising in elastic systems with varioius dampings, Adv. Differential Equations, 3(5) (1998), 643-686.

[20] Z.-Y. Liu and M. Renardy, A note on the equations of a thermoelastic plate, Appl. Math. Lett., 8(3) (1995), 1-6.

[21] Z. Liu and S. Zheng, Exponential stability of the Kirchhoff plate with thermal or viscoelastic damping, Quart. Appl. Math., 55(3) (1997), 551-564.

[22] J. E. Munoz Rivera and R. Racke, Smoothing properties, decay, and global existence of solutions to nonlinear coupled systems of thermoelastic type, SIAM J. Math. Anal., 26(6) (1995), 1547-1563.

[23] Y. Naito, On the $L_{p}-L_{q}$ maximal regularity for the linear thermoelastic plate equation in a bounded domain, Math. Methods Appl. Sci., 32(13) (2009), 1609-1637.

[24] Y. Naito and Y. Shibata, On the $L_{p}$ analytic semigroup associated with the linear thermoelastic plate equations in the half-space, J. Math. Soc. Japan, 61(4) (2009), 971-1011.

[25] K. Schade and Y. Shibata, On strong dynamics of compressible nematic liquid crystals, SIAM J. Math. Anal. 47 (5) (2015), 3963-3992.

[26] Y. Shibata, On the exponential decay of the energy of a linear thermoelastic plate, Mat. Apl. Comput., 13(2) (1994), 81-102.

[27] Y. Shibata, On the $\mathcal{R}$-boundedness of solution oeprators for the Stokes equations with free boundary condition, Differential Integral Equations, 27(3-4) (2014), 313-368.

[28] Y. Shibata, On some free boundary problem of the Navier-Stokes equations in the maximal $L_{p}-L_{q}$ regularity class, J. Differential Equations, 258(12) (2015), 4127-4155.

[29] Y. Shibata and S. Shimizu, On the $L_{p}-L_{q}$ maximal regularity of the Neumann problem for the Stokes equations in a bounded domain, J. Reine Angew. Math. 615 (2008), 157-209.

[30] Y. Shibata and S. Shimizu, On the maxial $L_{p}-L_{q}$ regularity of the Stokes problem with first order boundary condition; model problems, J. Math. Soc. Japan, 64 (2) (2012), 561-626.

[31] L. Weis, Operator-valued Fourier multiplier theorems and maximal $L_{p}$-regularity, Math. Ann., 319(4) (2001), 735-785. 